Barker, P.F., Camerlenghi, A., Acton, G.D., and Ramsay, A.T.S. (Eds.)

Proceedings of the Ocean Drilling Program, Scientific Results Volume 178

\section{Data Report: Grain-Size Data, SITES 1095, 1096, AND 1101, Antarctic Peninsula Continental Rise'}

\author{
Carol J. Pudsey²
}

\section{INTRODUCTION}

Sites 1095, 1096, and 1101 were drilled on the continental rise west of the Antarctic Peninsula (Fig. F1) to recover a continuous high-resolution record of Antarctic glaciation. Site 1095 is the subject of a short paper in this volume (Pudsey, Chap. 25, this volume), whereas mass accumulation rates at the three sites are described by Wolf-Welling (Chap. 15, this volume) and ice-rafted debris at Site 1101 is discussed by Cowan (Chap. 10, this volume) This report documents grain-size data (sand and fine fraction) and the proportion of biogenic silica for the upper $300 \mathrm{~m}$ at Site 1095, the upper $250 \mathrm{~m}$ at Site 1096, and the whole $220 \mathrm{~m}$ at Site 1101 .

\section{METHODS}

The cores were sampled approximately every $1.5 \mathrm{~m}$ (one sample per section). Selected intervals, where there was obvious lithologic cyclicity, were sampled more closely (every 0.2 to $0.4 \mathrm{~m}$ ). Sample size was $10 \mathrm{~cm}^{3}$.

Sample preparation was carried out at the British Antarctic Survey. Biogenic silica (in the form of diatoms, radiolarians, and rare silicoflagellates) was measured by point counting a smear slide made from each sample. This method is quick but tends to overestimate silica compared with the true weight percentage (Pudsey, 1993). For textural analysis, each dried sample of $\sim 5 \mathrm{~g}$ was wet sieved at $63 \mu \mathrm{m}(4 \phi)$ to measure sand percentage and the sand fraction weighed and retained. Fine-fraction size distribution down to $0.5 \mu \mathrm{m}(11 \phi)$ was measured on a Sedi-
F1. Location map, Sites 1095, 1096, and 1101, p. 6.

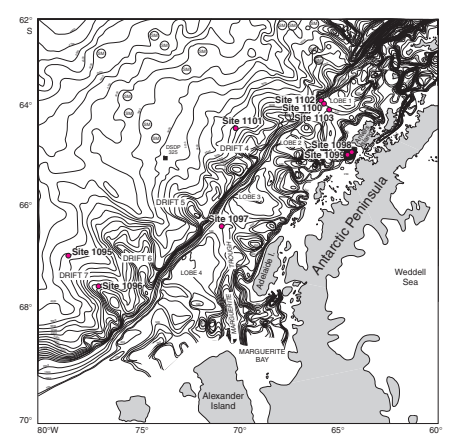

${ }^{1}$ Pudsey, C.J., 2001. Data report: Grain-size data, Sites 1095, 1096, and 1101, Antarctic Peninsula continental rise. In Barker, P.F., Camerlenghi, A., Acton, G.D., and Ramsay, A.T.S. (Eds.), Proc. ODP, Sci. Results, 178, 1-34

[Online]. Available from World Wide Web: <http://www-odp.tamu.edu/ publications/178_SR/VOLUME/ CHAPTERS/SR178_12.PDF>. [Cited YYYY-MM-DD]

${ }^{2}$ British Antarctic Survey, High Cross, Madingley Road, Cambridge CB3 OET, United Kingdom. cjp@pcmail.nerc-bas. ac.uk

Initial receipt: 16 August 2000 Acceptance: 25 April 2001

Web publication: 12 July 2001 
graph 5100 particle size analyzer at Royal Holloway and Bedford New College, University of London. The chosen Sedigraph output was the weight percentage within each $0.25-\phi$ interval from 4 to $11 \phi(63-0.5$ $\mu \mathrm{m})$. The very high clay content of most samples (commonly $>70 \%$ finer than $8 \phi(4 \mu \mathrm{m})$ and $>40 \%$ finer than the measurement limit of 11 $\phi)$ necessitated extrapolation of the fine end of the cumulative frequency curves to obtain values of $\phi_{16}$ to calculate the standard grain-size parameters of sorting $\left(\sigma_{\mathrm{G}}\right)$ and skewness $\left(\mathrm{Sk}_{\mathrm{G}}\right)$ (Folk, 1974). Modal size was measured to the nearest $0.1 \phi$ from each size-frequency histogram.

The data for each site are plotted against age using the sedimentation rate curves based mainly on magnetic reversal data of Shipboard Scientific Party (1999a, 1999b, 1999c).

\section{RESULTS}

The data are given in Tables T1, T2, T3, T4, T5, T6, T7, T8, and T9 and Figures F2, F3, F4, F5, F6, F7, F8, F9, and F10. Tables T1, T2, and T3 refer to Site 1095; downcore plots for this site are shown by Pudsey (Chap. 25, this volume).

\section{Site 1096}

Tables T4, T5, and T6 and Figures F2, F3, F4, and F5 refer to Site 1096. Diatom content is generally low $(<10 \%)$, reflecting dilution by the abundant fine-grained terrigenous supply to this site at the inshore end of Drift 7. The spikiness of the sand percentage and other grain-size data result from sampling the lithologic cycles that are present throughout the section (Shipboard Scientific Party, 1999b). Virtually all the sand is terrigenous. Clay content is generally very high $(70 \%-80 \%$ finer than $4 \mu \mathrm{m}$ ); the samples are poorly to very poorly sorted and positively (fine) skewed.

\section{Site 1101}

Tables T7, T8, and T9 and Figures F6, F7, F8, and F9 refer to Site 1101. Diatom content is generally low in the Quaternary but up to $38 \%$ in the upper Pliocene. Some of the sand in the samples from 50 to 135 meters below seafloor (mbsf) (0.7-2.0 Ma) comprises foraminiferal tests (Wolf-Welling, Chap. 15, this volume). The fine-fraction grain-size data (Fig. F8) emphasize the strong lithologic cyclicity, with silty clays and clayey silts alternating. Most median and many of the modal diameters are in the silt rather than the clay range. Sorting is poor and the samples are positively (fine) skewed.

None of the sites shows any marked trends in grain size downcore, but there are significant differences between sites. Figure F10 shows frequency histograms of median diameter and mode for each site. Note that not all samples have a well-defined mode. Approximately onethird of the samples from Sites 1095 and 1096 and 10\% of those from Site 1101 are polymodal or so poorly sorted that a mode could not be picked. At Site 1095, median diameter average $=9.65 \phi$ and most modes are near $8 \phi$ (silt/clay boundary), with a few in the silt range. At Site 1096 , median diameter average $=10.3 \phi$ and nearly all modes are $\sim 8 \phi$. Site 1101 has a much wider spread of median and modal diameters. It is likely that Site 1096 represents the lowest-energy hemipelagic environment with the weakest bottom currents. Some samples at Site 1095 in-
T1. Diatom percentage, Site 1095, p. 16.

T2. Grain-size summary, Site 1095, p. 17.

T3. Grain-size statistics, Site 1095, p. 21.

T4. Diatom percentage, Site 1096, p. 23.

T5. Grain-size summary, Site 1096, p. 24.

T6. Grain-size statistics, Site 1096, p. 27.

T7. Diatom percentage, Site 1101, p. 29.

T8. Grain-size summary, Site 1101, p. 30.

T9. Grain-size statistics, Site 1101, p. 33.

F2. Diatom percentage, Site 1096, p. 7 .

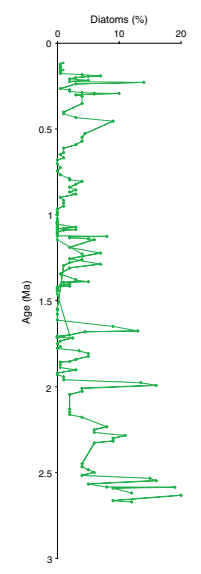




\section{C.J. Pudsey}

clude a silt component from turbidity currents (Pudsey, submitted, this volume). Site 1101 is a hemipelagic/contouritic section (Shipboard Scientific Party, 1999c), reflecting some sorting by bottom currents.

\section{SUMMARY}

Grain-size data for Sites 1095, 1096, and 1101 show the sections are fine-grained throughout, reflecting the low-energy depositional environment.

\section{ACKNOWLEDGMENTS}

The samples were collected during Leg 178 and I thank all the members of the sample shifts. Steve Moreton at BAS and Adrian Palmer at Royal Holloway are thanked for technical assistance. This work was supported by the Natural Environment Research Council, United Kingdom.
F3. Sand percentage, Site 1096, p. 8.

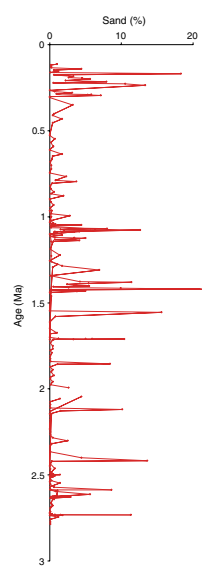

F4. Fine-fraction size distribution, Site 1096, p. 9.

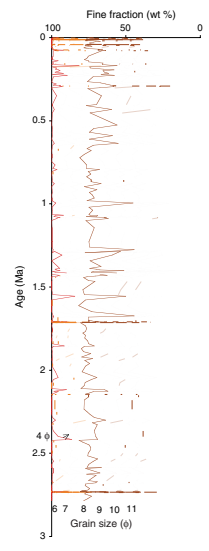

F5. Grain-size statistics, Site 1096, p. 10.

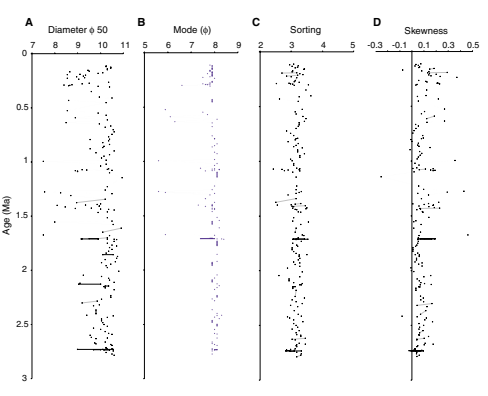




\section{C.J. Pudsey}

F6. Diatom percentage, Site 1101, p. 11.

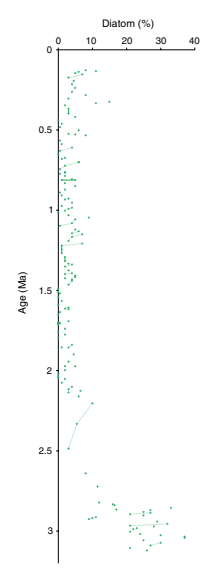

F7. Sand percentage, Site 1101, p. 12.

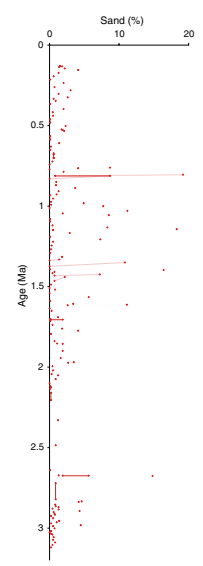

F8. Fine-fraction size distribution, Site 1101, p. 13.

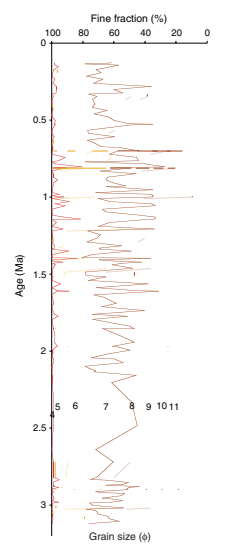

F9. Grain-size statistics, Site 1101, p. 14.

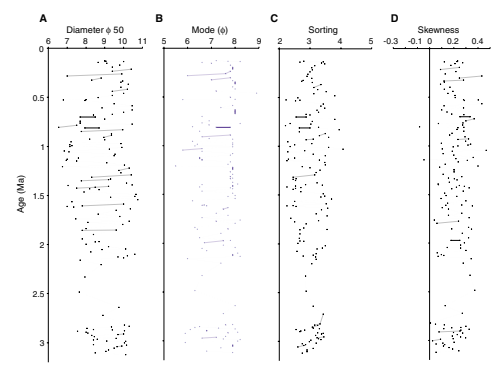

F10. Median diameter frequency histograms, p. 15.

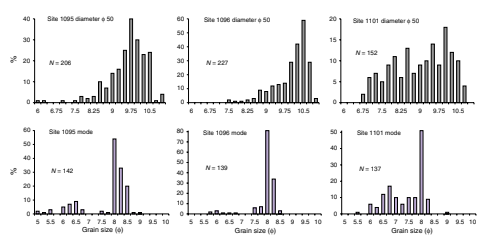




\section{REFERENCES}

Folk, R.L., 1974. Petrology of Sedimentary Rocks: Austin, TX (Hemphill Publ.).

Pudsey, C.J., 1993. Calibration of a point-counting technique for estimation of biogenic silica in marine sediments. J. Sediment. Petrol., 63:760-762.

Shipboard Scientific Party, 1999a. Site 1095. In Barker, P.F., Camerlenghi, A., Acton, G.D., et al., Proc. ODP, Init. Repts., 178, 1-173 [CD-ROM]. Available from: Ocean Drilling Program, Texas A\&M University, College Station, TX 77845-9547, U.S.A.

, 1999b. Site 1096. In Barker, P.F., Camerlenghi, A., Acton, G.D., et al., Proc. ODP, Init. Repts., 178, 1-144 [CD-ROM]. Available from: Ocean Drilling Program, Texas A\&M University, College Station, TX 77845-9547, U.S.A.

, 1999c. Site 1101. In Barker, P.F., Camerlenghi, A., Acton, G.D., et al., Proc. ODP, Init. Repts., 178, 1-83 [CD-ROM]. Available from: Ocean Drilling Program, Texas A\&M University, College Station, TX 77845-9547, U.S.A. 


\section{C.J. Pudsey}

DATA Report: Grain-Size DATA

Figure F1. Location map of Sites 1095, 1096, and 1101.

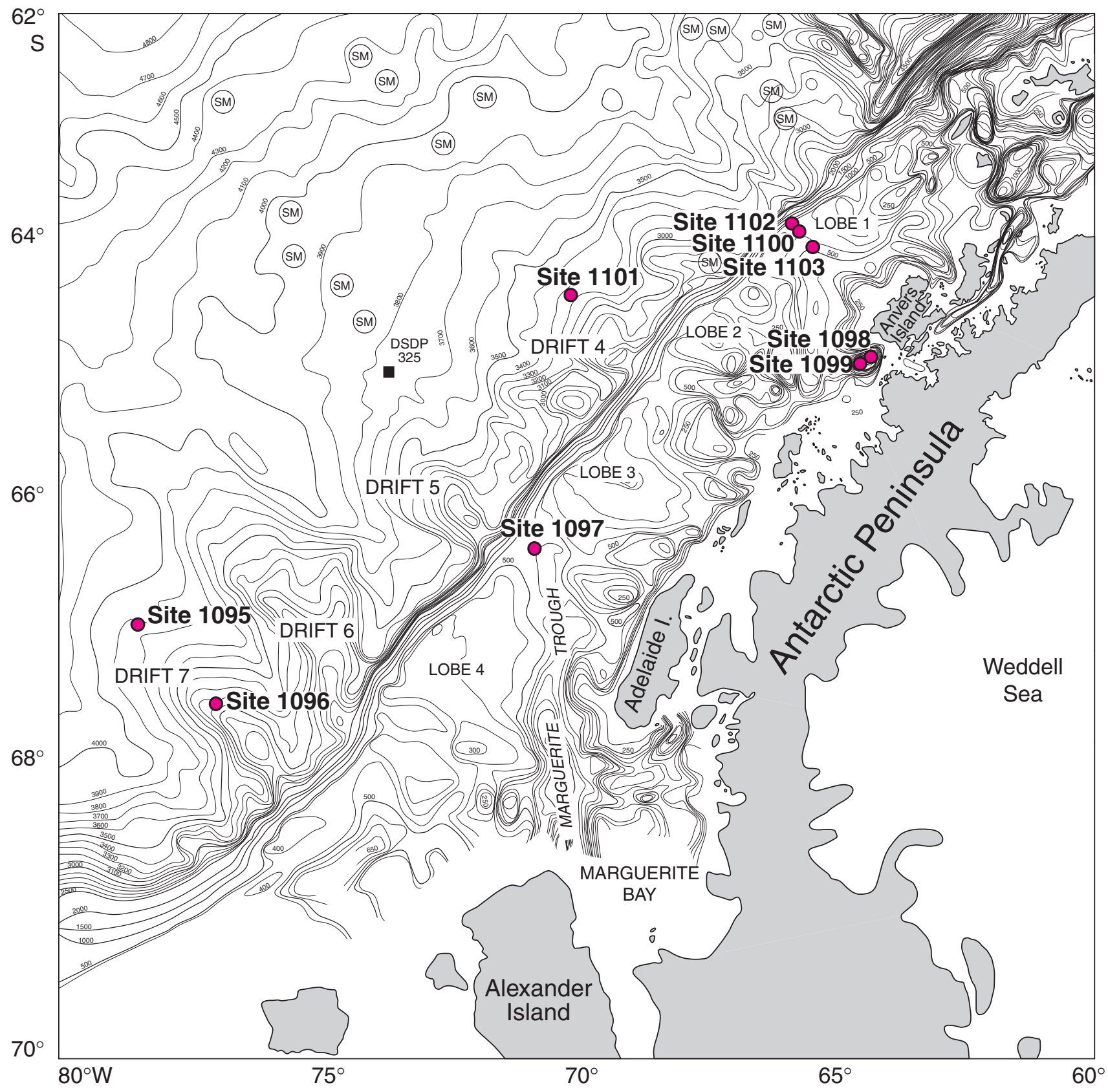




\section{C.J. Pudsey}

Figure F2. Diatom percentage for Site 1096.

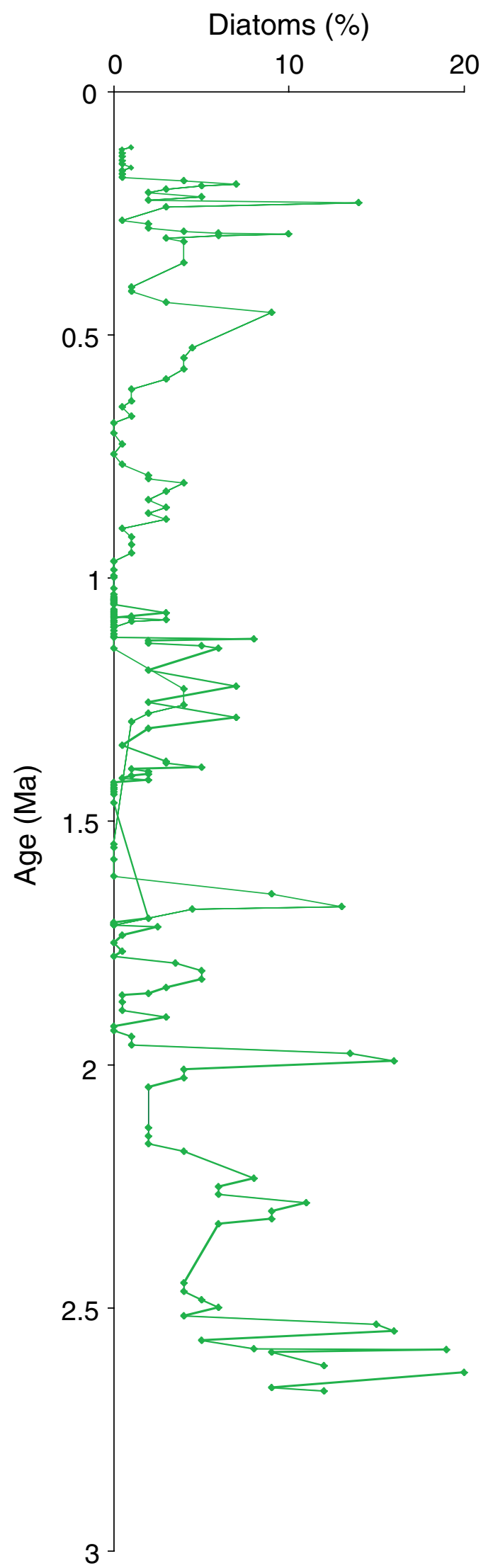


C.J. Pudsey

DATA Report: Grain-Size DAta

8

Figure F3. Sand percentage for Site 1096.

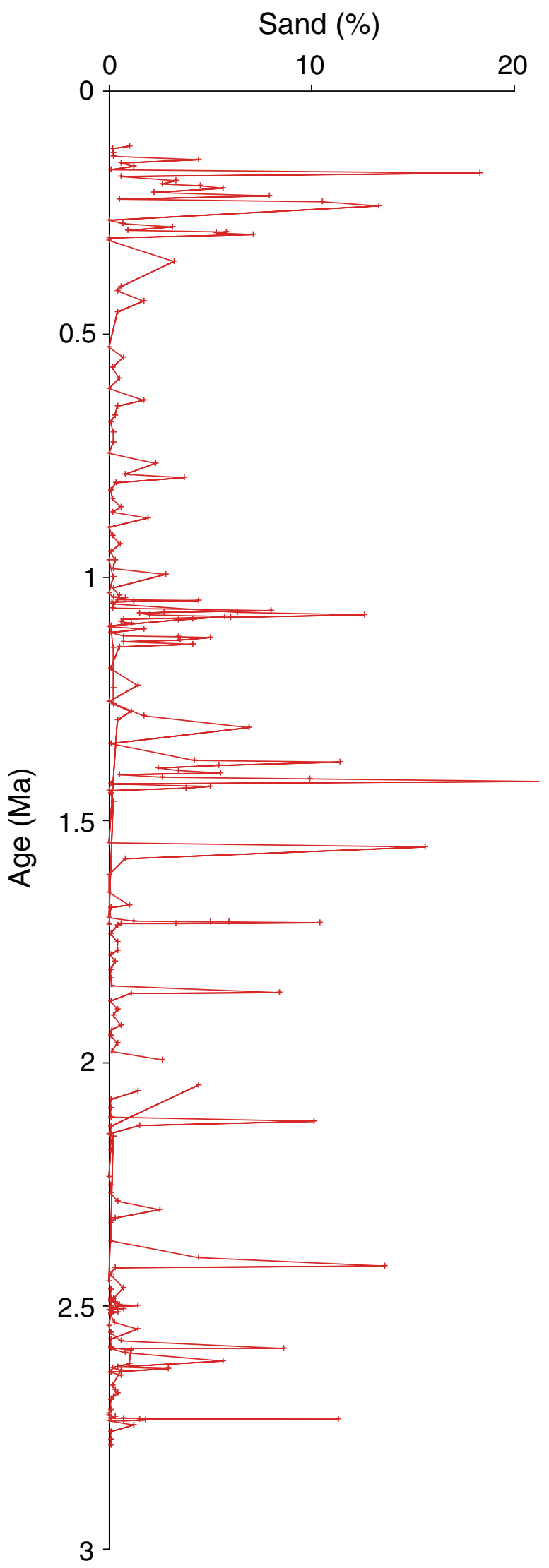




\section{C.J. Pudsey}

DAta Report: Grain-Size Data

Figure F4. Fine-fraction ( $>4 \phi$ ) size distribution (weight percentage within 1- $\phi$ intervals) for Site 1096.

Fine fraction (wt \%)

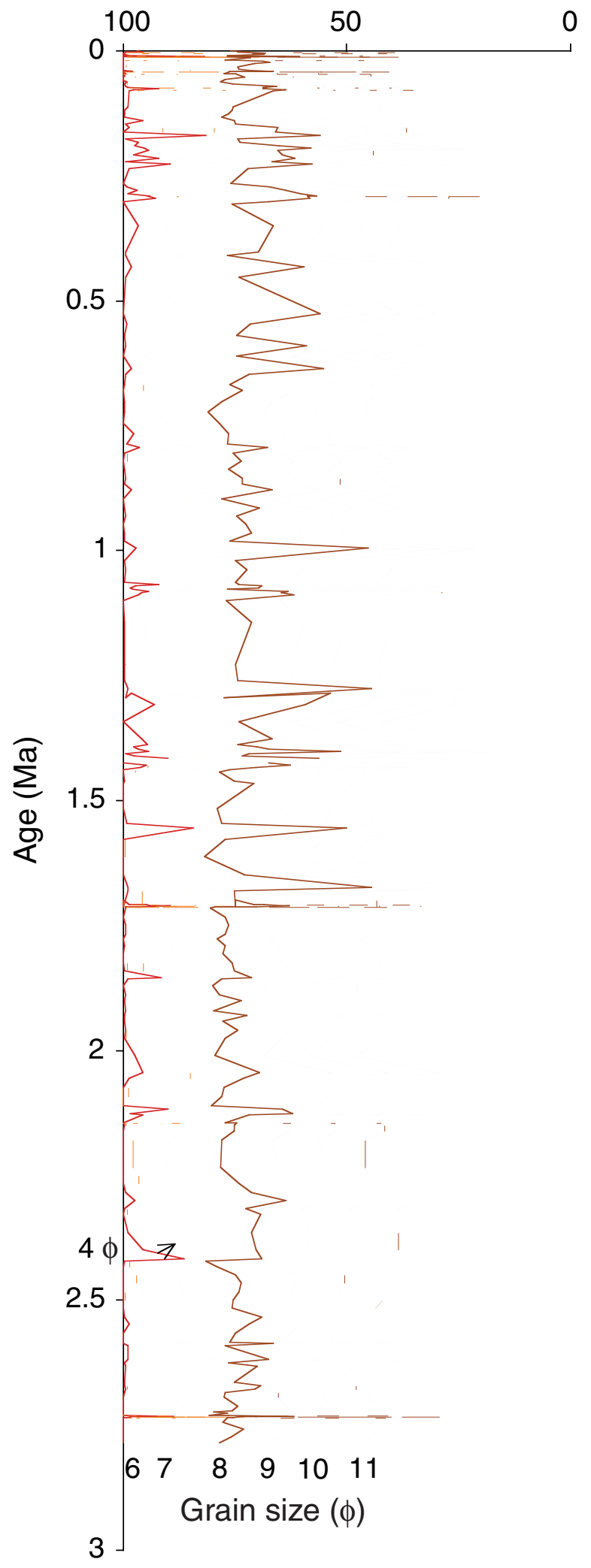


Figure F5. Site 1096 grain-size statistics. A. Median diameter. B. Modal size. Not all samples have a well-defined mode. C. Sorting. D. Skewness.

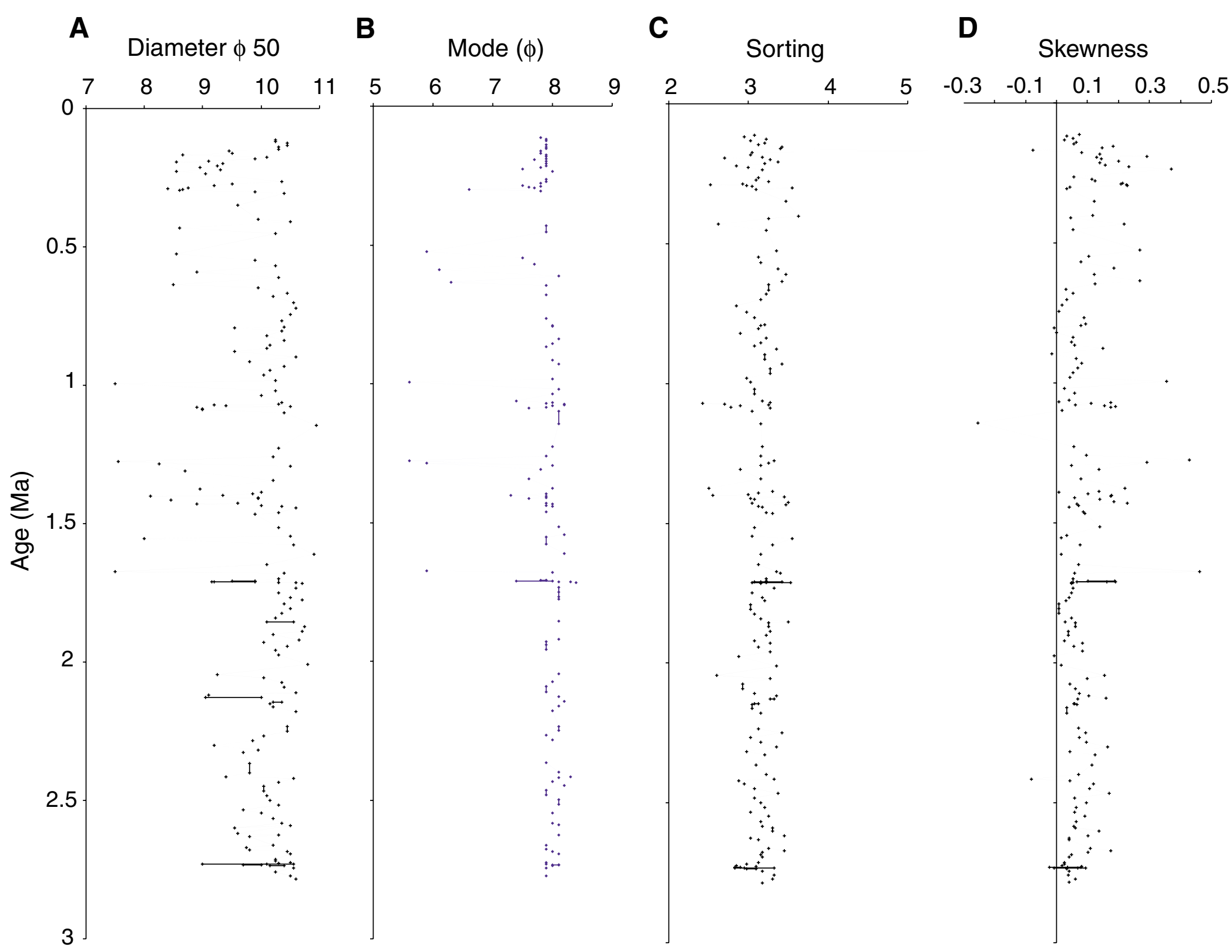




\section{C.J. PUDSEY}

DAta RePort: GRAIN-Size DATA

Figure F6. Diatom percentage for Site 1101.

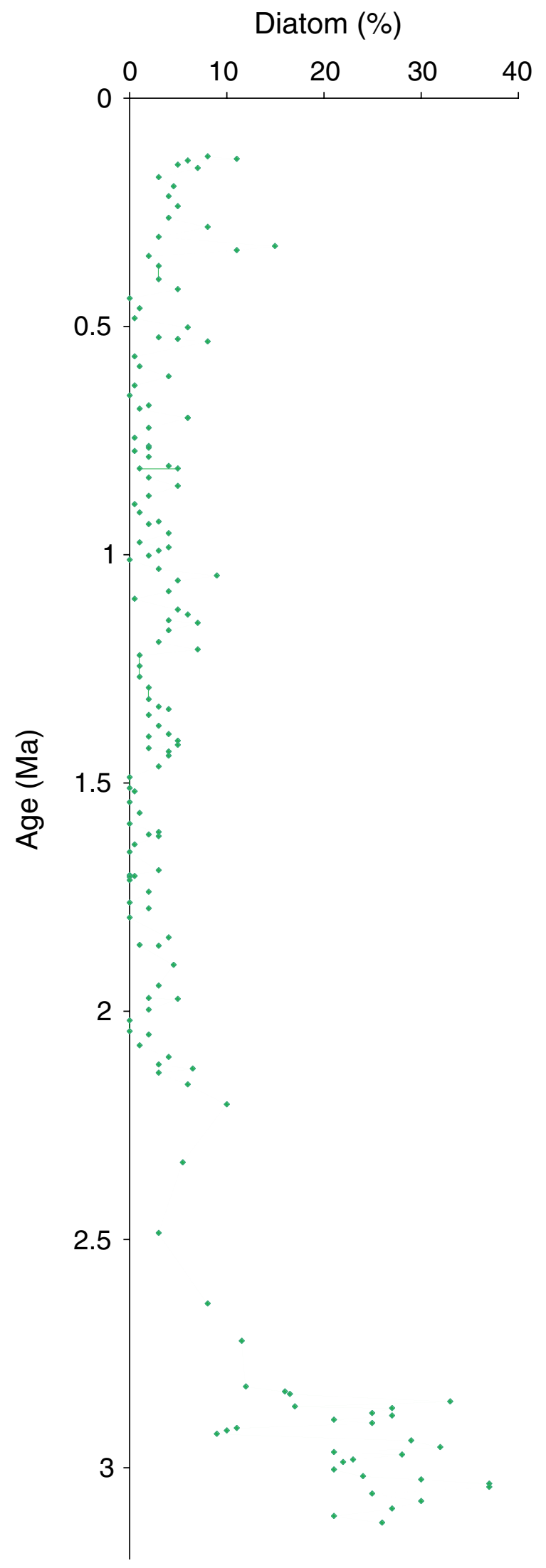




\section{C.J. PUdSEY}

DAta Report: GRAIn-Size DAta

Figure F7. Sand percentage for Site 1101.

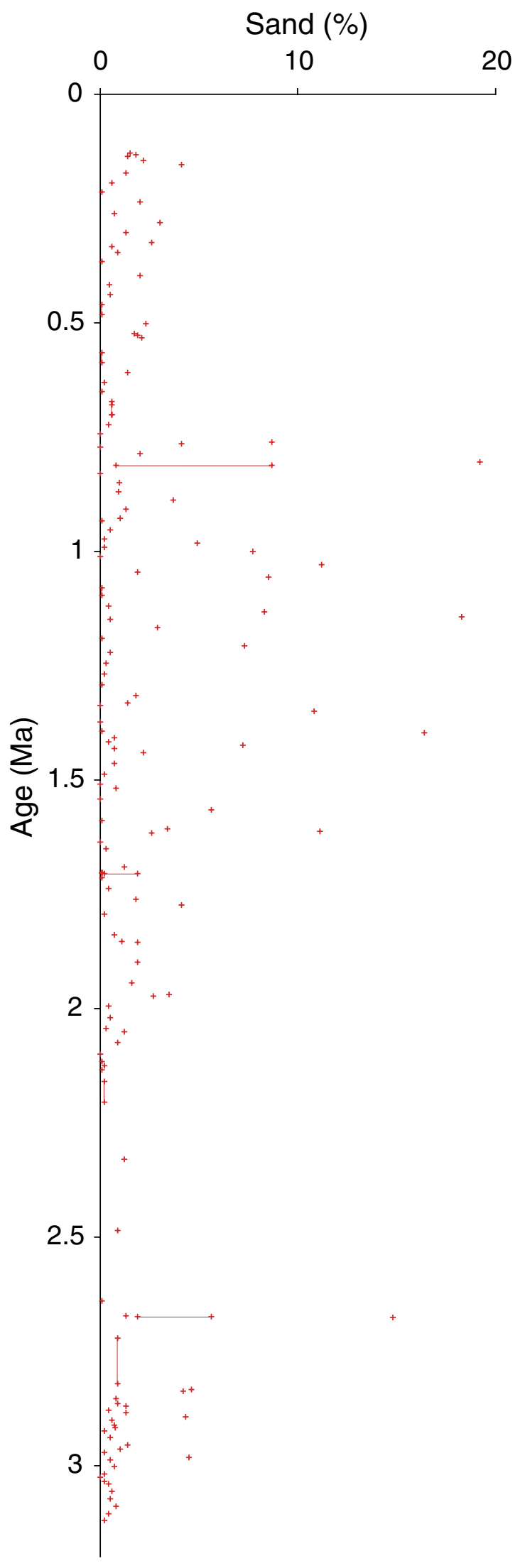




\section{C.J. Pudsey}

DAta Report: Grain-Size Data

Figure F8. Fine-fraction ( $>4 \phi$ ) size distribution (weight percentage within 1- $\phi$ intervals) for Site 1101.

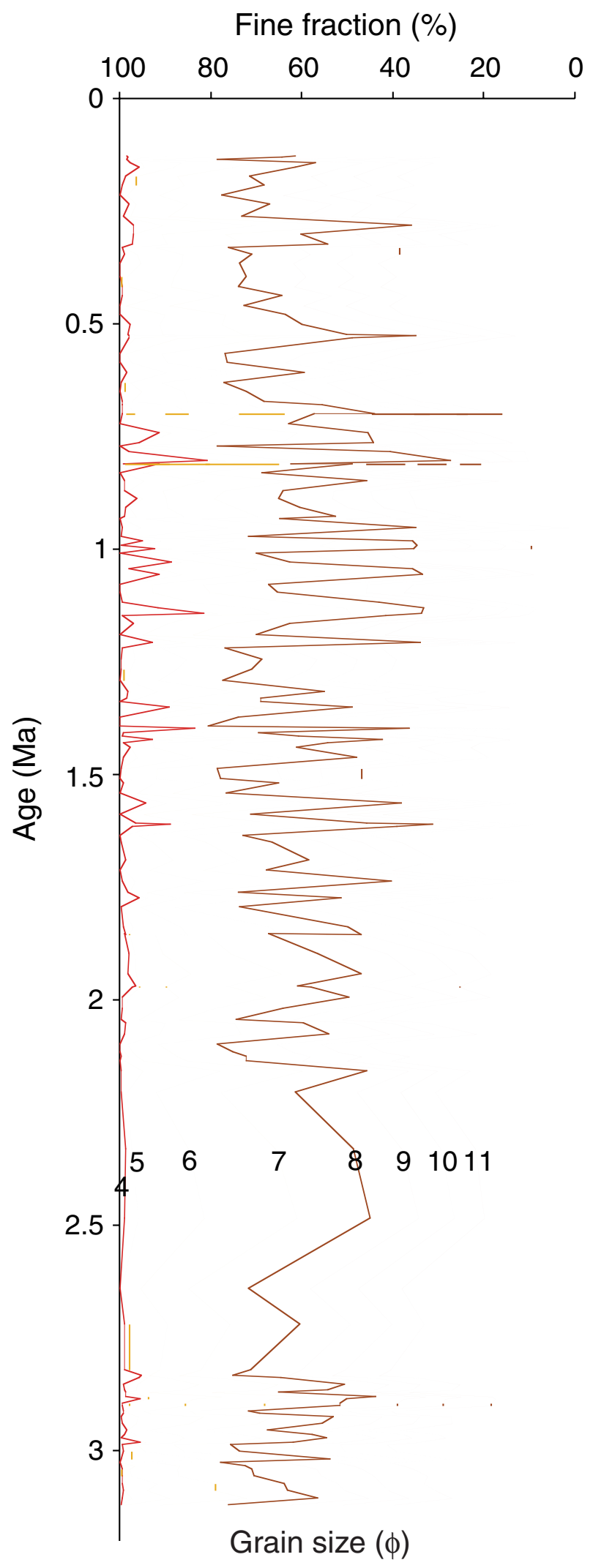


Figure F9. Site 1101 grain-size statistics. A. Median diameter. B. Modal size. Not all samples have a well-defined mode. C. Sorting. D. Skewness.

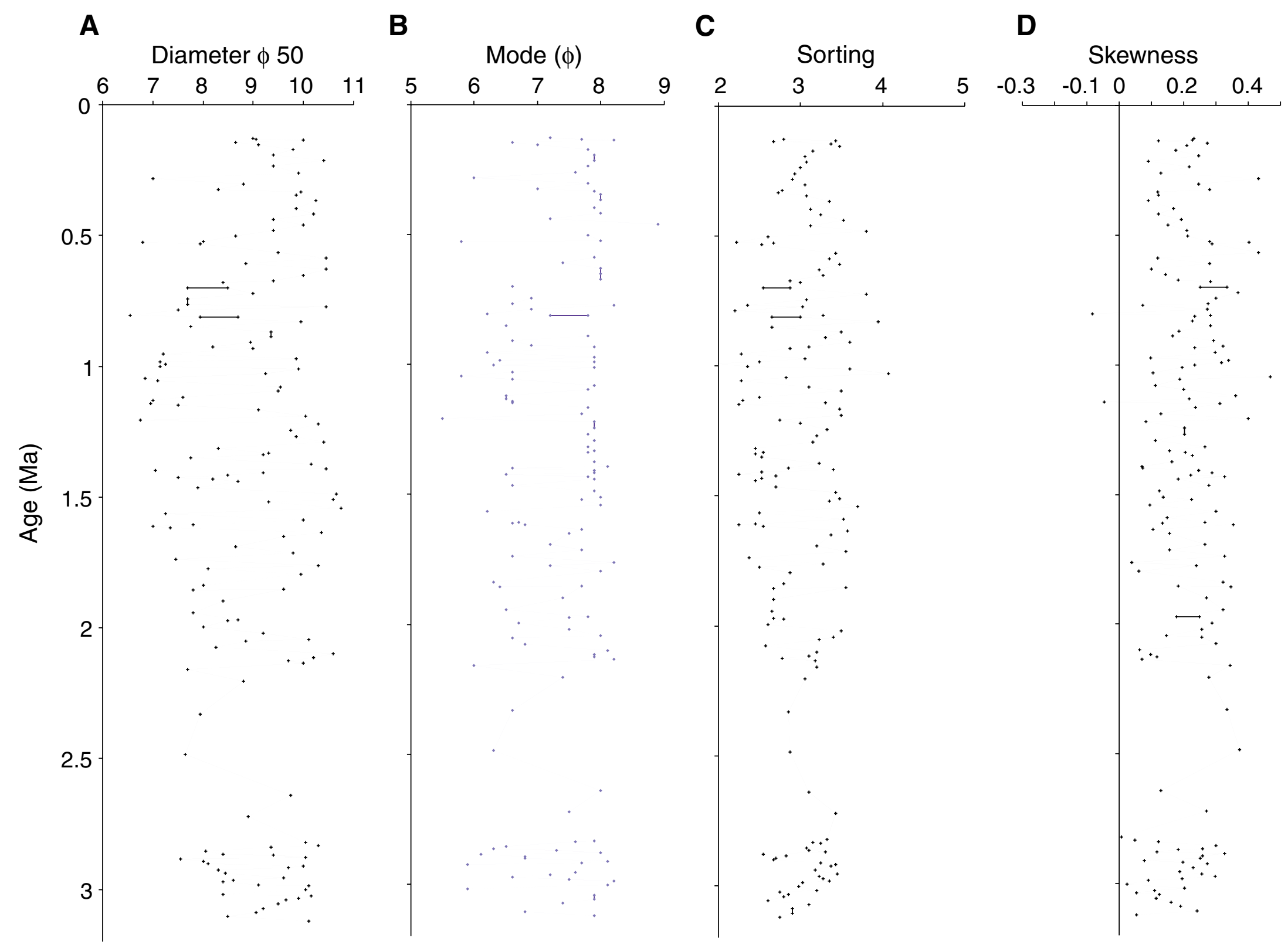


Figure F10. Frequency histograms of median diameter and mode for the three sites.
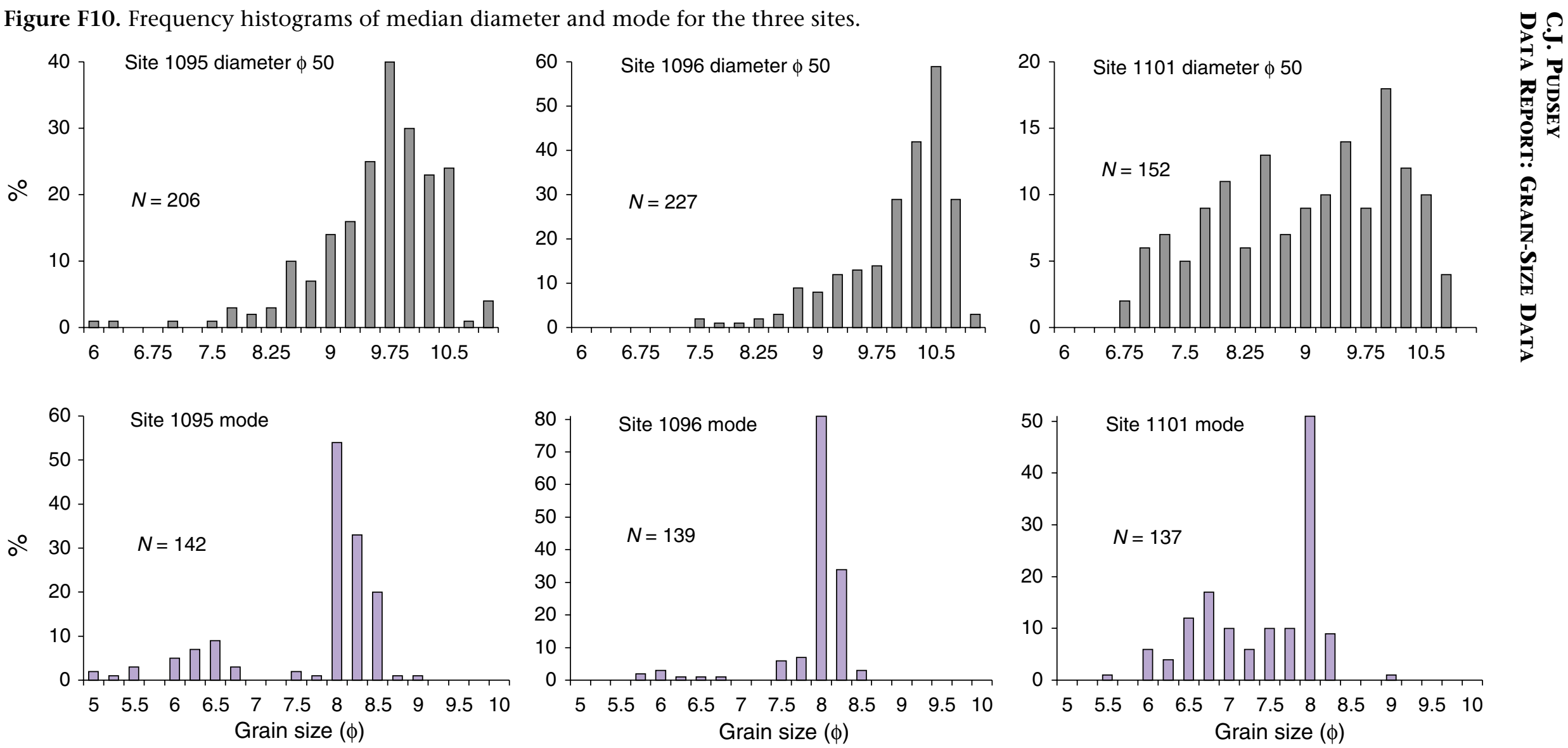
C.J. Pudsey

Table T1. Diatom percentages, Site 1095.

\begin{tabular}{|c|c|c|}
\hline $\begin{array}{l}\text { Depth } \\
\text { (mbsf) }\end{array}$ & $\begin{array}{l}\text { Age } \\
\text { (Ma) }\end{array}$ & $\begin{array}{c}\text { Diatoms } \\
(\%)\end{array}$ \\
\hline 0.00 & 0.000 & \\
\hline 0.90 & 0.041 & 2 \\
\hline 1.66 & 0.076 & 5 \\
\hline 2.12 & 0.097 & 10 \\
\hline 2.59 & 0.118 & 1 \\
\hline 3.41 & 0.155 & 2 \\
\hline 4.41 & 0.201 & 9 \\
\hline 4.59 & 0.209 & 34 \\
\hline 4.69 & 0.214 & 14 \\
\hline 4.79 & 0.218 & 5 \\
\hline 4.94 & 0.225 & 2 \\
\hline 5.09 & 0.232 & 10.5 \\
\hline 5.32 & 0.242 & 6 \\
\hline 5.49 & 0.250 & 8 \\
\hline 5.59 & 0.255 & 12 \\
\hline 5.69 & 0.259 & 20 \\
\hline 5.80 & 0.264 & 21 \\
\hline 5.90 & 0.269 & 17 \\
\hline 6.04 & 0.275 & 5 \\
\hline 6.34 & 0.289 & 4 \\
\hline 6.64 & 0.303 & 4 \\
\hline 6.94 & 0.316 & 4.5 \\
\hline 7.25 & 0.330 & 6 \\
\hline 7.54 & 0.344 & 10 \\
\hline 7.84 & 0.357 & 13.6 \\
\hline 8.19 & 0.373 & 0.5 \\
\hline 10.02 & 0.456 & 0.5 \\
\hline 10.20 & 0.465 & 3 \\
\hline 11.36 & 0.518 & 3 \\
\hline 12.33 & 0.562 & 0 \\
\hline 15.09 & 0.687 & 0 \\
\hline 15.56 & 0.709 & 9 \\
\hline 16.52 & 0.753 & 6 \\
\hline 17.67 & 0.802 & 7 \\
\hline 19.33 & 0.859 & 0 \\
\hline 22.49 & 0.968 & 0 \\
\hline 24.01 & 1.000 & 0 \\
\hline 25.00 & 1.012 & 0 \\
\hline 25.95 & 1.024 & 7 \\
\hline 26.99 & 1.036 & 2 \\
\hline 27.76 & 1.046 & 10 \\
\hline 28.54 & 1.055 & 3 \\
\hline 31.10 & 1.101 & 1 \\
\hline 31.50 & 1.110 & 2 \\
\hline 31.90 & 1.119 & 0.5 \\
\hline 32.30 & 1.128 & 2 \\
\hline 32.80 & 1.140 & 0 \\
\hline 33.10 & 1.147 & 0 \\
\hline 33.53 & 1.156 & 0 \\
\hline 33.88 & 1.164 & 0 \\
\hline 34.27 & 1.173 & 0 \\
\hline 34.70 & 1.183 & 0 \\
\hline 35.00 & 1.190 & 0 \\
\hline 35.39 & 1.199 & 0 \\
\hline 35.80 & 1.208 & 0 \\
\hline 36.09 & 1.215 & 2 \\
\hline 36.34 & 1.221 & 0 \\
\hline 40.76 & 1.322 & 0 \\
\hline 41.80 & 1.345 & 0 \\
\hline 43.69 & 1.388 & 0 \\
\hline 44.91 & 1.416 & 0 \\
\hline 46.78 & 1.459 & 6 \\
\hline 47.95 & 1.486 & 2 \\
\hline 50.06 & 1.534 & 8 \\
\hline 51.39 & 1.564 & 4 \\
\hline 51.90 & 1.576 & 8 \\
\hline
\end{tabular}

\begin{tabular}{|c|c|c|}
\hline $\begin{array}{l}\text { Depth } \\
\text { (mbsf) }\end{array}$ & $\begin{array}{l}\text { Age } \\
(\mathrm{Ma})\end{array}$ & $\begin{array}{c}\text { Diatoms } \\
(\%)\end{array}$ \\
\hline 52.89 & 1.599 & 6 \\
\hline 54.39 & 1.633 & 7 \\
\hline 55.89 & 1.667 & 4.5 \\
\hline 57.39 & 1.701 & 8 \\
\hline 58.79 & 1.733 & 8 \\
\hline 60.20 & 1.766 & 6 \\
\hline 61.70 & 1.842 & 6 \\
\hline 63.20 & 1.925 & 10 \\
\hline 64.10 & 1.975 & 8 \\
\hline 65.60 & 2.058 & 9 \\
\hline 67.10 & 2.140 & 7 \\
\hline 68.00 & 2.190 & 7 \\
\hline 69.31 & 2.262 & 12 \\
\hline 71.00 & 2.356 & 12 \\
\hline 71.87 & 2.404 & 7 \\
\hline 73.27 & 2.481 & 9 \\
\hline 74.81 & 2.566 & 11 \\
\hline 77.02 & 2.688 & 15 \\
\hline 78.77 & 2.784 & 17 \\
\hline 79.02 & 2.798 & 14 \\
\hline 79.14 & 2.805 & 10 \\
\hline 79.27 & 2.812 & 22 \\
\hline 79.44 & 2.821 & 14 \\
\hline 79.59 & 2.830 & 12 \\
\hline 79.81 & 2.842 & 14 \\
\hline 80.07 & 2.856 & 20 \\
\hline 80.45 & 2.877 & 21 \\
\hline 80.83 & 2.898 & 17 \\
\hline 81.19 & 2.918 & 20 \\
\hline 81.34 & 2.926 & 16 \\
\hline 81.47 & 2.933 & 8 \\
\hline 81.65 & 2.943 & 15 \\
\hline 81.81 & 2.952 & 23 \\
\hline 82.05 & 2.965 & 15 \\
\hline 82.82 & 3.008 & 17 \\
\hline 84.67 & 3.079 & 27 \\
\hline 87.04 & 3.152 & 25 \\
\hline 84.98 & 3.089 & 14 \\
\hline 88.85 & 3.207 & 17 \\
\hline 90.24 & 3.250 & 22 \\
\hline 91.92 & 3.302 & 21 \\
\hline 94.20 & 3.372 & 25 \\
\hline 95.36 & 3.407 & 17 \\
\hline 96.75 & 3.450 & 20 \\
\hline 98.19 & 3.494 & 22 \\
\hline 99.87 & 3.546 & 18 \\
\hline 104.09 & 3.654 & 21 \\
\hline 107.72 & 3.740 & 24 \\
\hline 109.23 & 3.776 & 23 \\
\hline 111.40 & 3.828 & 45 \\
\hline 114.28 & 3.896 & 27 \\
\hline 116.51 & 3.949 & 22 \\
\hline 118.34 & 3.993 & 27 \\
\hline 120.24 & 4.038 & 28 \\
\hline 122.08 & 4.082 & 25 \\
\hline 126.44 & 4.183 & 35 \\
\hline 133.38 & 4.270 & 39 \\
\hline 135.17 & 4.296 & 52 \\
\hline 138.95 & 4.415 & 48 \\
\hline 142.87 & 4.515 & 25 \\
\hline 144.22 & 4.540 & 29 \\
\hline 145.38 & 4.562 & 32 \\
\hline 146.67 & 4.586 & 27 \\
\hline 148.16 & 4.614 & 41 \\
\hline 149.07 & 4.633 & 26 \\
\hline 150.60 & 4.668 & 20 \\
\hline
\end{tabular}

\begin{tabular}{|c|c|c|}
\hline $\begin{array}{l}\text { Depth } \\
\text { (mbsf) }\end{array}$ & $\begin{array}{l}\text { Age } \\
(\mathrm{Ma})\end{array}$ & $\begin{array}{c}\text { Diatoms } \\
\text { (\%) }\end{array}$ \\
\hline 152.10 & 4.701 & 25 \\
\hline 153.59 & 4.735 & 0 \\
\hline 155.09 & 4.769 & 14 \\
\hline 156.60 & 4.803 & 19 \\
\hline 158.10 & 4.836 & 16 \\
\hline 158.69 & 4.850 & 10 \\
\hline 159.90 & 4.877 & 28 \\
\hline 161.40 & 4.911 & 27 \\
\hline 163.00 & 4.947 & 29 \\
\hline 165.92 & 5.013 & 14 \\
\hline 167.41 & 5.058 & 51 \\
\hline 168.19 & 5.079 & 24 \\
\hline 169.30 & 5.109 & 22 \\
\hline 170.80 & 5.149 & 30 \\
\hline 172.30 & 5.189 & 23 \\
\hline 173.20 & 5.213 & 45 \\
\hline 173.80 & 5.229 & 16 \\
\hline 175.30 & 5.270 & 20 \\
\hline 176.80 & 5.310 & 23 \\
\hline 177.63 & 5.332 & 19 \\
\hline 179.27 & 5.259 & 30 \\
\hline 185.24 & 5.384 & 14 \\
\hline 188.31 & 5.448 & 10 \\
\hline 192.90 & 5.543 & 20 \\
\hline 198.13 & 5.652 & 10 \\
\hline 201.79 & 5.728 & 18 \\
\hline 205.19 & 5.799 & 15 \\
\hline 207.80 & 5.854 & 16 \\
\hline 210.36 & 5.907 & 18 \\
\hline 215.19 & 6.012 & 14 \\
\hline 216.38 & 6.037 & 15 \\
\hline 217.80 & 6.068 & 14 \\
\hline 226.73 & 6.178 & 8 \\
\hline 235.99 & 6.244 & 10 \\
\hline 236.20 & 6.246 & 11 \\
\hline 236.39 & 6.247 & 30 \\
\hline 236.59 & 6.248 & 21 \\
\hline 236.79 & 6.250 & 47 \\
\hline 237.00 & 6.251 & 3 \\
\hline 237.19 & 6.253 & 10 \\
\hline 237.39 & 6.254 & 10 \\
\hline 237.99 & 6.258 & 10 \\
\hline 238.57 & 6.263 & 11 \\
\hline 239.08 & 6.266 & 11 \\
\hline 239.29 & 6.268 & 12 \\
\hline 239.68 & 6.274 & 7 \\
\hline 240.09 & 6.280 & 7 \\
\hline 240.74 & 6.291 & 7 \\
\hline 242.53 & 6.320 & 23 \\
\hline 244.03 & 6.344 & 13 \\
\hline 246.30 & 6.381 & 14 \\
\hline 248.64 & 6.419 & 6 \\
\hline 250.49 & 6.449 & 10 \\
\hline 252.33 & 6.479 & 18 \\
\hline 264.91 & 6.643 & 8 \\
\hline 268.10 & 6.678 & 12 \\
\hline 269.44 & 6.692 & 14 \\
\hline 271.44 & 6.714 & 7 \\
\hline 274.88 & 6.751 & 12 \\
\hline 276.67 & 6.770 & 15 \\
\hline 281.14 & 6.818 & 10.5 \\
\hline 284.74 & 6.857 & 15 \\
\hline 292.70 & 6.944 & 12 \\
\hline 297.23 & 7.003 & 13 \\
\hline 300.25 & 7.042 & 10 \\
\hline
\end{tabular}


C.J. Pudsey

DATA Report: Grain-Size DATA

Table T2. Fine-fraction ( $>4 \phi)$ size distribution, Site 1095. (See table notes. Continued on next three pages.)

\begin{tabular}{|c|c|c|c|c|c|c|c|c|c|c|}
\hline \multirow[b]{2}{*}{ Core } & \multirow{2}{*}{$\begin{array}{l}\text { Depth } \\
\text { (mbsf) }\end{array}$} & \multirow{2}{*}{$\begin{array}{l}\text { Age } \\
\text { (Ma) }\end{array}$} & \multicolumn{8}{|c|}{ Grain size $(\phi)$} \\
\hline & & & 4 & 5 & 6 & 7 & 8 & 9 & 10 & 11 \\
\hline \multicolumn{11}{|c|}{ 178-1095A- } \\
\hline $1 \mathrm{H}$ & 0.90 & 0.041 & 99.86 & 99.86 & 98.94 & 92.71 & 80.85 & 67.56 & 56.11 & 43.64 \\
\hline $1 \mathrm{H}$ & 1.66 & 0.076 & 98.20 & 97.02 & 94.17 & 87.79 & 75.22 & 59.71 & 47.33 & 34.17 \\
\hline $1 \mathrm{H}$ & 2.12 & 0.097 & 94.30 & 92.69 & 89.76 & 79.37 & 61.80 & 46.21 & 33.92 & 23.53 \\
\hline $1 \mathrm{H}$ & 2.59 & 0.118 & 87.70 & 85.93 & 80.27 & 71.15 & 59.74 & 48.32 & 38.85 & 28.76 \\
\hline $1 \mathrm{H}$ & 3.41 & 0.155 & 99.20 & 99.20 & 98.08 & 93.69 & 83.40 & 69.33 & 57.91 & 47.51 \\
\hline $1 \mathrm{H}$ & 4.41 & 0.201 & 93.50 & 91.54 & 88.73 & 78.07 & 62.83 & 48.25 & 36.56 & 25.53 \\
\hline \multicolumn{11}{|c|}{ 178-1095D- } \\
\hline $1 \mathrm{H}$ & 4.59 & 0.209 & 92.10 & 91.08 & 87.21 & 77.80 & 62.66 & 48.54 & 37.19 & 27.32 \\
\hline $1 \mathrm{H}$ & 4.69 & 0.214 & 92.80 & 92.80 & 91.56 & 84.39 & 68.62 & 54.57 & 43.87 & 35.65 \\
\hline $1 \mathrm{H}$ & 4.79 & 0.218 & 92.40 & 92.12 & 88.75 & 81.45 & 68.43 & 55.33 & 45.03 & 35.57 \\
\hline $1 \mathrm{H}$ & 4.94 & 0.225 & 90.20 & 88.57 & 86.76 & 81.42 & 71.47 & 59.26 & 49.13 & 40.53 \\
\hline $1 \mathrm{H}$ & 5.09 & 0.232 & 90.70 & 89.24 & 87.50 & 81.47 & 69.21 & 56.96 & 46.54 & 37.30 \\
\hline $1 \mathrm{H}$ & 5.32 & 0.242 & 96.00 & 94.64 & 91.73 & 83.96 & 70.37 & 56.69 & 45.72 & 36.40 \\
\hline $1 \mathrm{H}$ & 5.49 & 0.250 & 97.70 & 96.32 & 91.70 & 81.66 & 65.82 & 51.75 & 42.60 & 33.65 \\
\hline $1 \mathrm{H}$ & 5.59 & 0.255 & 98.00 & 97.50 & 95.12 & 84.09 & 67.49 & 52.78 & 42.14 & 32.80 \\
\hline $1 \mathrm{H}$ & 5.69 & 0.259 & 95.20 & 95.20 & 92.01 & 81.46 & 64.63 & 50.12 & 39.47 & 29.31 \\
\hline $1 \mathrm{H}$ & 5.80 & 0.264 & 94.30 & 93.91 & 92.93 & 85.50 & 71.31 & 56.64 & 45.98 & 36.00 \\
\hline $1 \mathrm{H}$ & 5.90 & 0.269 & 95.00 & 94.41 & 93.63 & 85.97 & 71.94 & 57.80 & 47.40 & 37.00 \\
\hline $1 \mathrm{H}$ & 6.04 & 0.275 & 96.20 & 95.33 & 92.51 & 85.12 & 73.66 & 60.83 & 50.24 & 40.52 \\
\hline $1 \mathrm{H}$ & 6.34 & 0.289 & 97.00 & 96.90 & 96.00 & 89.10 & 77.40 & 63.70 & 53.60 & 42.10 \\
\hline $1 \mathrm{H}$ & 6.64 & 0.303 & 97.40 & 97.01 & 92.99 & 84.48 & 72.34 & 59.52 & 49.34 & 40.53 \\
\hline $1 \mathrm{H}$ & 6.94 & 0.316 & 84.50 & 83.98 & 83.37 & 78.53 & 67.88 & 55.41 & 45.97 & 37.14 \\
\hline $1 \mathrm{H}$ & 7.25 & 0.330 & 96.40 & 95.04 & 91.74 & 83.78 & 70.29 & 56.79 & 46.60 & 36.70 \\
\hline $1 \mathrm{H}$ & 7.54 & 0.344 & 97.10 & 95.84 & 91.85 & 81.92 & 66.84 & 53.80 & 43.69 & 34.25 \\
\hline $1 \mathrm{H}$ & 7.84 & 0.357 & 96.00 & 94.45 & 91.05 & 81.15 & 66.10 & 53.19 & 43.00 & 34.46 \\
\hline $1 \mathrm{H}$ & 8.19 & 0.373 & 97.50 & 96.33 & 93.00 & 84.87 & 72.73 & 60.40 & 49.92 & 40.92 \\
\hline $2 \mathrm{H}$ & 10.02 & 0.456 & 97.70 & 96.91 & 94.15 & 87.26 & 74.56 & 61.75 & 51.21 & 41.46 \\
\hline $2 \mathrm{H}$ & 10.39 & 0.473 & 95.30 & 95.30 & 94.02 & 87.81 & 75.69 & 62.78 & 53.42 & 43.17 \\
\hline $2 \mathrm{H}$ & 10.84 & 0.494 & 90.50 & 90.50 & 89.48 & 85.20 & 72.10 & 56.49 & 44.60 & 33.08 \\
\hline $2 \mathrm{H}$ & 11.89 & 0.542 & 94.50 & 93.83 & 90.38 & 82.23 & 69.49 & 56.35 & 46.10 & 36.90 \\
\hline $2 \mathrm{H}$ & 12.34 & 0.562 & 96.90 & 96.90 & 95.39 & 88.85 & 75.87 & 62.08 & 52.63 & 42.36 \\
\hline $2 \mathrm{H}$ & 12.92 & 0.589 & 99.10 & 99.10 & 96.38 & 89.13 & 77.65 & 64.46 & 54.79 & 44.62 \\
\hline $2 \mathrm{H}$ & 13.30 & 0.606 & 97.70 & 96.82 & 94.07 & 84.44 & 71.48 & 58.82 & 49.19 & 40.16 \\
\hline $2 \mathrm{H}$ & 13.84 & 0.631 & & 98.50 & & & 75.52 & & 52.05 & 42.56 \\
\hline $2 \mathrm{H}$ & 14.39 & 0.656 & 99.00 & 98.60 & 96.09 & 87.97 & 75.13 & 62.29 & 52.46 & 43.13 \\
\hline \multicolumn{11}{|c|}{ 178-1095A- } \\
\hline $3 \mathrm{H}$ & & & & & & & & 5 & .05 & \\
\hline $3 \mathrm{H}$ & 15.11 & 0.688 & 96.40 & 96.01 & 93.39 & 86.20 & 74.92 & 62.00 & 51.80 & 42.18 \\
\hline $3 \mathrm{H}$ & 15.56 & 0.709 & 97.40 & 97.40 & 96.10 & 87.57 & 71.12 & 54.17 & 41.63 & 29.79 \\
\hline $3 \mathrm{H}$ & 16.52 & 0.753 & 97.80 & 97.80 & 97.40 & 93.41 & 80.55 & 65.50 & 53.74 & 43.97 \\
\hline $3 \mathrm{H}$ & 17.67 & 0.802 & 95.30 & 95.30 & 94.42 & 88.04 & 73.73 & 57.85 & 45.39 & 32.94 \\
\hline $3 \mathrm{H}$ & 19.33 & 0.859 & 97.80 & 97.41 & 94.85 & 84.63 & 72.64 & 61.14 & 51.41 & 42.17 \\
\hline $4 \mathrm{H}$ & 22.49 & 0.968 & 97.70 & 97.50 & 94.36 & 87.89 & 75.92 & 63.96 & 53.66 & 45.02 \\
\hline $4 \mathrm{H}$ & 24.01 & 1.000 & 93.50 & 92.18 & 86.34 & 77.19 & 66.45 & 55.61 & 46.56 & 38.08 \\
\hline $4 \mathrm{H}$ & 25.00 & 1.012 & 95.50 & 95.50 & 94.63 & 92.59 & 83.94 & 70.82 & 59.75 & 50.13 \\
\hline $4 \mathrm{H}$ & 25.95 & 1.024 & 97.50 & 96.42 & 93.29 & 84.77 & 68.52 & 52.57 & 39.84 & 27.12 \\
\hline $4 \mathrm{H}$ & 26.99 & 1.036 & 87.70 & 85.45 & 82.25 & 75.84 & 63.46 & 50.82 & 40.69 & 32.38 \\
\hline $4 \mathrm{H}$ & 27.76 & 1.046 & 93.20 & 91.51 & 86.06 & 75.07 & 59.00 & 44.35 & 33.45 & 23.30 \\
\hline $4 \mathrm{H}$ & 28.54 & 1.055 & 95.30 & 93.96 & 89.29 & 80.70 & 66.68 & 52.85 & 42.26 & 32.34 \\
\hline $5 \mathrm{H}$ & 31.10 & 1.101 & 96.20 & 96.10 & 95.70 & 88.56 & 69.10 & 49.84 & 36.34 & 24.22 \\
\hline $5 \mathrm{H}$ & 31.50 & 1.110 & 90.20 & 88.83 & 84.90 & 73.68 & 56.15 & 40.81 & 29.58 & 21.36 \\
\hline $5 \mathrm{H}$ & 31.90 & 1.119 & 91.10 & 89.17 & 86.04 & 77.40 & 64.53 & 50.84 & 39.44 & 29.23 \\
\hline $5 \mathrm{H}$ & 32.30 & 1.128 & 92.10 & 92.10 & 90.84 & 81.65 & 65.98 & 50.40 & 38.70 & 28.83 \\
\hline $5 \mathrm{H}$ & 32.80 & 1.140 & 99.30 & 99.10 & 95.49 & 86.36 & 74.63 & 63.09 & 52.76 & 43.83 \\
\hline $5 \mathrm{H}$ & 33.10 & 1.147 & 96.40 & 96.01 & 93.07 & 84.85 & 73.79 & 61.66 & 50.89 & 41.30 \\
\hline $5 \mathrm{H}$ & 33.53 & 1.156 & 97.30 & 95.63 & 89.66 & 77.80 & 62.42 & 49.19 & 38.70 & 29.98 \\
\hline $5 \mathrm{H}$ & 33.88 & 1.164 & 98.90 & 98.60 & 96.39 & 86.45 & 73.20 & 60.44 & 49.90 & 38.96 \\
\hline $5 \mathrm{H}$ & 34.27 & 1.173 & 99.30 & 98.90 & 97.50 & 91.39 & 80.08 & 66.67 & 55.06 & 44.54 \\
\hline $5 \mathrm{H}$ & 34.70 & 1.183 & 97.50 & 97.01 & 95.23 & 88.71 & 76.76 & 63.62 & 52.65 & 41.98 \\
\hline $5 \mathrm{H}$ & 35.00 & 1.190 & 95.40 & 95.40 & 93.65 & 87.31 & 77.37 & 65.29 & 54.96 & 44.73 \\
\hline $5 \mathrm{H}$ & 35.39 & 1.199 & 95.20 & 94.53 & 90.96 & 83.83 & 73.23 & 60.51 & 49.14 & 38.74 \\
\hline $5 \mathrm{H}$ & 35.80 & 1.208 & 99.70 & 99.40 & 95.45 & 86.14 & 72.88 & 59.42 & 48.99 & 38.46 \\
\hline $5 \mathrm{H}$ & 36.09 & 1.215 & 93.30 & 91.98 & 84.42 & 69.69 & 51.47 & 36.83 & 27.01 & 19.74 \\
\hline $5 \mathrm{H}$ & 36.34 & 1.221 & 99.20 & 98.19 & 88.17 & 73.49 & 61.44 & 50.31 & 40.89 & 32.59 \\
\hline $6 \mathrm{H}$ & 40.76 & 1.322 & 93.80 & 91.91 & 89.56 & 84.47 & 76.27 & 65.05 & 54.87 & 44.68 \\
\hline $6 \mathrm{H}$ & 41.80 & 1.345 & 85.50 & 84.45 & 80.24 & 74.10 & 64.80 & 53.49 & 44.37 & 35.78 \\
\hline
\end{tabular}


C.J. Pudsey

DATA Report: Grain-Size DATA

Table T2 (continued).

\begin{tabular}{|c|c|c|c|c|c|c|c|c|c|c|}
\hline \multirow[b]{2}{*}{ Core } & \multirow{2}{*}{$\begin{array}{l}\text { Depth } \\
\text { (mbsf) }\end{array}$} & \multirow{2}{*}{$\begin{array}{l}\text { Age } \\
(\mathrm{Ma})\end{array}$} & \multicolumn{8}{|c|}{ Grain size $(\phi)$} \\
\hline & & & 4 & 5 & 6 & 7 & 8 & 9 & 10 & 11 \\
\hline $6 \mathrm{H}$ & 43.69 & 1.388 & 90.90 & 88.22 & 82.59 & 75.29 & 63.83 & 52.01 & 43.14 & 34.09 \\
\hline $6 \mathrm{H}$ & 44.91 & 1.416 & 99.90 & 99.90 & 97.48 & 90.22 & 78.43 & 65.22 & 54.54 & 44.05 \\
\hline $6 \mathrm{H}$ & 46.78 & 1.459 & 86.70 & 86.17 & 83.45 & 78.61 & 69.38 & 56.89 & 45.90 & 34.38 \\
\hline $6 \mathrm{H}$ & 47.95 & 1.486 & 99.85 & 99.75 & 96.62 & 89.35 & 77.23 & 64.41 & 53.61 & 42.61 \\
\hline $7 \mathrm{H}$ & 50.06 & 1.534 & 99.80 & 96.02 & 84.29 & 68.37 & 56.02 & 45.31 & 36.74 & 27.70 \\
\hline $7 \mathrm{H}$ & 51.39 & 1.564 & 99.80 & 90.67 & 73.50 & 59.08 & 48.35 & 39.87 & 32.91 & 25.81 \\
\hline $7 \mathrm{H}$ & 51.90 & 1.576 & 99.90 & 99.70 & 96.89 & 87.78 & 74.85 & 61.92 & 51.10 & 40.08 \\
\hline $7 \mathrm{H}$ & 52.89 & 1.599 & 89.30 & 87.94 & 84.05 & 76.26 & 64.85 & 52.62 & 42.02 & 31.70 \\
\hline \multicolumn{11}{|c|}{ 178-1095D- } \\
\hline $6 \mathrm{H}$ & 53.41 & 1.599 & 99.80 & 99.80 & 97.79 & 90.65 & 78.19 & 64.42 & 53.37 & 42.81 \\
\hline $6 \mathrm{H}$ & 53.67 & 1.599 & 99.80 & 98.59 & 94.27 & 86.13 & 75.38 & 62.81 & 52.97 & 43.52 \\
\hline $6 \mathrm{H}$ & 53.86 & 1.599 & 85.90 & 84.35 & 81.50 & 77.53 & 69.34 & 58.04 & 48.38 & 38.38 \\
\hline $6 \mathrm{H}$ & 54.07 & 1.599 & 88.60 & 87.00 & 84.60 & 80.16 & 70.47 & 58.83 & 49.50 & 40.70 \\
\hline $6 \mathrm{H}$ & 54.26 & 1.599 & 95.50 & 94.54 & 91.96 & 86.31 & 77.32 & 66.31 & 56.94 & 48.61 \\
\hline \multicolumn{11}{|c|}{ 178-1095A- } \\
\hline 7H & 54.39 & 1.633 & 97.00 & 96.70 & 90.96 & 78.67 & 66.38 & 54.69 & 44.98 & 35.87 \\
\hline \multicolumn{11}{|c|}{ 178-1095D- } \\
\hline $6 \mathrm{H}$ & 54.46 & 1.635 & 98.80 & 98.01 & 95.83 & 90.39 & 80.88 & 68.70 & 58.51 & 49.10 \\
\hline $6 \mathrm{H}$ & 54.67 & 1.639 & 99.50 & 99.20 & 96.19 & 88.05 & 76.71 & 63.96 & 53.41 & 42.87 \\
\hline $6 \mathrm{H}$ & 54.86 & 1.644 & 99.70 & 99.70 & 98.88 & 93.74 & 82.14 & 68.08 & 57.19 & 46.92 \\
\hline $6 \mathrm{H}$ & 55.06 & 1.648 & 98.60 & 98.60 & 98.08 & 91.90 & 79.85 & 65.63 & 54.40 & 43.58 \\
\hline $6 \mathrm{H}$ & 55.33 & 1.654 & 99.70 & 99.20 & 95.47 & 85.59 & 71.78 & 59.17 & 49.30 & 39.72 \\
\hline \multicolumn{11}{|c|}{ 178-1095A- } \\
\hline $7 \mathrm{H}$ & 55.89 & 1.667 & 99.85 & 99.85 & 96.72 & 89.95 & 77.82 & 63.47 & 52.25 & 41.84 \\
\hline $7 \mathrm{H}$ & 57.39 & 1.701 & 96.60 & 90.34 & 74.19 & 56.22 & 44.01 & 35.83 & 29.47 & 23.92 \\
\hline $7 \mathrm{H}$ & 58.79 & 1.733 & 84.20 & 83.01 & 81.82 & 76.21 & 67.53 & 56.05 & 45.76 & 35.30 \\
\hline $8 \mathrm{H}$ & 60.20 & 1.766 & 93.85 & 93.18 & 87.08 & 77.35 & 66.57 & 55.13 & 45.59 & 35.10 \\
\hline $8 \mathrm{H}$ & 61.70 & 1.842 & 99.60 & 99.40 & 89.41 & 73.60 & 61.27 & 49.85 & 41.70 & 32.62 \\
\hline $8 \mathrm{H}$ & 63.20 & 1.925 & 88.20 & 86.50 & 82.55 & 75.74 & 65.16 & 53.69 & 44.10 & 34.33 \\
\hline $8 \mathrm{H}$ & 64.10 & 1.975 & 99.70 & 95.70 & 82.18 & 69.88 & 60.15 & 49.39 & 40.99 & 32.38 \\
\hline $8 \mathrm{H}$ & 65.60 & 2.058 & 87.60 & 86.61 & 82.11 & 72.58 & 59.00 & 45.51 & 34.90 & 23.92 \\
\hline $8 \mathrm{H}$ & 67.10 & 2.140 & 95.70 & 86.68 & 74.62 & 67.56 & 59.62 & 50.30 & 43.14 & 35.50 \\
\hline $8 \mathrm{H}$ & 68.00 & 2.190 & 99.40 & 97.69 & 92.76 & 81.49 & 68.01 & 56.14 & 47.29 & 38.53 \\
\hline $9 \mathrm{H}$ & 69.31 & 2.262 & 99.80 & 99.08 & 89.34 & 72.52 & 57.64 & 46.46 & 37.54 & 27.69 \\
\hline $9 \mathrm{H}$ & 71.00 & 2.356 & 99.50 & 99.09 & 91.53 & 78.46 & 66.09 & 54.55 & 44.95 & 35.86 \\
\hline $9 \mathrm{H}$ & 71.87 & 2.404 & 71.90 & 69.92 & 64.56 & 56.57 & 46.74 & 37.64 & 29.64 & 21.79 \\
\hline $9 \mathrm{H}$ & 73.27 & 2.481 & 99.80 & 98.29 & 92.13 & 77.10 & 63.88 & 52.88 & 43.69 & 34.41 \\
\hline $9 \mathrm{H}$ & 74.81 & 2.566 & 99.40 & 98.29 & 90.80 & 82.31 & 72.50 & 61.38 & 51.77 & 41.66 \\
\hline $9 \mathrm{H}$ & 77.02 & 2.688 & 98.60 & 97.40 & 88.68 & 77.86 & 68.34 & 57.62 & 47.90 & 37.48 \\
\hline $10 \mathrm{H}$ & 78.77 & 2.784 & 99.90 & 98.57 & 80.21 & 57.64 & 45.13 & 37.13 & 30.56 & 24.62 \\
\hline $10 \mathrm{H}$ & 79.02 & 2.798 & 99.70 & 99.40 & 96.97 & 89.08 & 76.95 & 63.40 & 52.28 & 40.75 \\
\hline $10 \mathrm{H}$ & 79.14 & 2.805 & 81.90 & 81.57 & 78.81 & 72.31 & 61.38 & 49.37 & 38.78 & 28.94 \\
\hline $10 \mathrm{H}$ & 79.27 & 2.812 & 91.40 & 89.53 & 84.77 & 73.66 & 57.70 & 42.48 & 31.00 & 20.54 \\
\hline $10 \mathrm{H}$ & 79.44 & 2.821 & 93.70 & 92.09 & 88.19 & 80.03 & 66.07 & 51.17 & 39.87 & 28.48 \\
\hline $10 \mathrm{H}$ & 79.59 & 2.830 & 92.00 & 89.87 & 84.69 & 77.56 & 68.86 & 56.83 & 44.52 & 34.80 \\
\hline $10 \mathrm{H}$ & 79.81 & 2.842 & 99.40 & 96.34 & 81.15 & 64.94 & 53.22 & 43.43 & 35.07 & 27.12 \\
\hline $10 \mathrm{H}$ & 80.07 & 2.856 & 99.90 & 99.90 & 97.67 & 87.54 & 73.76 & 60.18 & 47.11 & 37.69 \\
\hline $10 \mathrm{H}$ & 80.45 & 2.877 & 99.75 & 99.75 & 94.79 & 82.23 & 68.86 & 56.00 & 45.77 & 35.85 \\
\hline $10 \mathrm{H}$ & 80.83 & 2.898 & 99.65 & 99.55 & 95.23 & 86.68 & 75.01 & 62.44 & 51.28 & 42.13 \\
\hline $10 \mathrm{H}$ & 81.19 & 2.918 & 91.70 & 90.95 & 88.06 & 80.51 & 66.14 & 49.91 & 37.78 & 28.55 \\
\hline $10 \mathrm{H}$ & 81.34 & 2.926 & 97.50 & 96.91 & 92.49 & 87.19 & 78.45 & 66.18 & 54.69 & 44.68 \\
\hline $10 \mathrm{H}$ & 81.47 & 2.933 & 86.20 & 85.67 & 80.75 & 71.25 & 55.59 & 40.02 & 28.23 & 18.47 \\
\hline $10 \mathrm{H}$ & 81.65 & 2.943 & 96.60 & 95.12 & 89.91 & 78.60 & 64.43 & 49.68 & 38.17 & 28.13 \\
\hline $10 \mathrm{H}$ & 81.81 & 2.952 & 98.50 & 96.22 & 90.26 & 80.03 & 66.92 & 53.92 & 43.49 & 34.16 \\
\hline $10 \mathrm{H}$ & 82.05 & 2.965 & 99.40 & 98.50 & 93.58 & 85.34 & 73.09 & 59.54 & 48.80 & 37.95 \\
\hline $10 \mathrm{H}$ & 82.82 & 3.008 & 99.30 & 98.90 & 97.59 & 88.24 & 75.38 & 62.62 & 52.16 & 41.61 \\
\hline $10 \mathrm{H}$ & 84.67 & 3.079 & 99.65 & 99.15 & 93.09 & 78.77 & 66.57 & 55.57 & 45.59 & 36.31 \\
\hline $10 \mathrm{H}$ & 87.04 & 3.152 & 96.40 & 95.32 & 90.03 & 81.40 & 68.65 & 53.05 & 40.11 & 29.52 \\
\hline $10 \mathrm{H}$ & 88.85 & 3.207 & 95.80 & 93.60 & 91.69 & 87.58 & 79.26 & 67.12 & 54.98 & 42.93 \\
\hline $10 \mathrm{H}$ & 90.24 & 3.250 & 99.60 & 99.30 & 97.29 & 88.97 & 74.32 & 61.59 & 50.55 & 40.62 \\
\hline $10 \mathrm{H}$ & 91.92 & 3.302 & 95.60 & 94.07 & 89.97 & 82.80 & 69.91 & 56.54 & 45.84 & 34.86 \\
\hline $10 \mathrm{H}$ & 94.20 & 3.372 & 95.90 & 95.13 & 91.96 & 83.42 & 70.94 & 58.46 & 48.67 & 38.97 \\
\hline $10 \mathrm{H}$ & 95.36 & 3.407 & 99.60 & 98.69 & 93.53 & 84.84 & 71.59 & 56.42 & 44.29 & 33.47 \\
\hline $10 \mathrm{H}$ & 96.75 & 3.450 & 92.30 & 90.44 & 80.31 & 65.90 & 53.63 & 43.69 & 35.69 & 28.44 \\
\hline $10 \mathrm{H}$ & 98.19 & 3.494 & 99.70 & 99.70 & 95.97 & 87.09 & 76.19 & 64.28 & 54.29 & 44.50 \\
\hline $10 \mathrm{H}$ & 99.87 & 3.546 & 99.70 & 97.42 & 93.15 & 87.50 & 78.47 & 65.87 & 54.86 & 43.65 \\
\hline
\end{tabular}


C.J. Pudsey

DATA Report: Grain-Size DATA

Table T2 (continued).

\begin{tabular}{|c|c|c|c|c|c|c|c|c|c|c|}
\hline \multirow{2}{*}{ Core } & \multirow{2}{*}{$\begin{array}{l}\text { Depth } \\
\text { (mbsf) }\end{array}$} & \multirow{2}{*}{$\begin{array}{c}\text { Age } \\
(\mathrm{Ma})\end{array}$} & \multicolumn{8}{|c|}{ Grain size $(\phi)$} \\
\hline & & & 4 & 5 & 6 & 7 & 8 & 9 & 10 & 11 \\
\hline \multicolumn{11}{|c|}{ 178-1095B- } \\
\hline $3 \mathrm{H}$ & 104.09 & 3.654 & 97.60 & 95.20 & 88.31 & 79.02 & 66.73 & 55.44 & 45.65 & 35.66 \\
\hline $3 \mathrm{H}$ & 107.72 & 3.740 & 99.90 & 98.50 & 93.62 & 81.06 & 67.60 & 55.43 & 45.06 & 35.09 \\
\hline $3 \mathrm{H}$ & 109.23 & 3.776 & 97.10 & 95.62 & 89.03 & 80.77 & 70.54 & 58.14 & 46.83 & 36.20 \\
\hline $3 \mathrm{H}$ & 111.40 & 3.828 & 99.60 & 98.19 & 92.87 & 83.03 & 69.98 & 58.03 & 47.19 & 37.25 \\
\hline $4 \mathrm{H}$ & 114.28 & 3.896 & 100.00 & 97.90 & 93.21 & 81.44 & 68.76 & 56.59 & 46.21 & 36.33 \\
\hline $4 \mathrm{H}$ & 116.51 & 3.949 & 99.90 & 98.09 & 85.84 & 68.17 & 54.12 & 44.18 & 35.74 & 28.31 \\
\hline $4 \mathrm{H}$ & 118.34 & 3.993 & 95.40 & 94.63 & 91.94 & 87.23 & 77.61 & 63.95 & 51.93 & 39.33 \\
\hline $4 \mathrm{H}$ & 120.24 & 4.038 & 98.00 & 98.00 & 96.89 & 92.97 & 82.02 & 66.64 & 54.18 & 41.91 \\
\hline $5 \mathrm{H}$ & 122.08 & 4.082 & 99.80 & 98.40 & 90.79 & 77.68 & 64.26 & 52.05 & 41.94 & 31.63 \\
\hline $5 \mathrm{H}$ & 126.44 & 4.183 & 98.10 & 96.82 & 94.65 & 87.86 & 75.74 & 60.97 & 47.38 & 34.37 \\
\hline $6 \mathrm{H}$ & 133.38 & 4.270 & 100.00 & 99.90 & 97.49 & 88.98 & 75.45 & 62.42 & 51.30 & 40.48 \\
\hline $6 \mathrm{H}$ & 135.17 & 4.296 & 95.70 & 94.63 & 90.55 & 82.68 & 69.86 & 54.02 & 40.90 & 29.92 \\
\hline $6 \mathrm{H}$ & 138.95 & 4.415 & 99.80 & 98.90 & 93.21 & 80.84 & 67.86 & 55.29 & 45.21 & 35.33 \\
\hline $7 \mathrm{H}$ & 142.87 & 4.515 & 100.00 & 99.59 & 91.03 & 75.54 & 63.30 & 52.29 & 43.12 & 34.35 \\
\hline $7 \mathrm{H}$ & 144.22 & 4.540 & 100.00 & 99.39 & 95.36 & 87.59 & 74.27 & 60.24 & 49.65 & 38.45 \\
\hline $7 \mathrm{H}$ & 145.38 & 4.562 & 100.00 & 99.31 & 77.20 & 47.11 & 31.60 & 23.73 & 18.52 & 14.00 \\
\hline $7 \mathrm{H}$ & 146.67 & 4.586 & 100.00 & 97.52 & 89.50 & 75.15 & 60.79 & 49.21 & 40.00 & 30.59 \\
\hline $7 \mathrm{H}$ & 148.16 & 4.614 & 100.00 & 97.88 & 94.46 & 87.70 & 75.81 & 61.39 & 49.29 & 38.41 \\
\hline $7 \mathrm{H}$ & 149.07 & 4.633 & 94.40 & 94.02 & 86.31 & 69.47 & 54.81 & 44.35 & 36.45 & 28.07 \\
\hline $8 \mathrm{H}$ & 150.60 & 4.668 & 93.40 & 93.21 & 92.73 & 89.16 & 77.59 & 61.21 & 46.65 & 34.89 \\
\hline $8 \mathrm{H}$ & 152.10 & 4.701 & 95.50 & 92.23 & 84.44 & 76.84 & 65.49 & 52.80 & 41.93 & 31.06 \\
\hline $8 \mathrm{H}$ & 153.59 & 4.735 & 55.50 & 31.27 & 9.55 & 4.60 & 3.67 & 2.91 & 2.39 & 1.51 \\
\hline $8 \mathrm{H}$ & 155.09 & 4.769 & 99.10 & 88.10 & 52.55 & 34.93 & 28.01 & 22.71 & 18.43 & 15.18 \\
\hline $8 \mathrm{H}$ & 156.60 & 4.803 & 98.80 & 95.52 & 86.18 & 75.44 & 64.71 & 53.57 & 44.43 & 34.79 \\
\hline $8 \mathrm{H}$ & 158.10 & 4.836 & 98.80 & 98.70 & 95.60 & 86.19 & 73.97 & 61.36 & 50 & 39.64 \\
\hline $8 \mathrm{H}$ & 158.69 & 4.850 & 99.20 & 98.80 & 97.51 & 91.15 & 80.51 & 66.60 & 53.28 & 38.96 \\
\hline $9 \mathrm{H}$ & 159.90 & 4.877 & 99.80 & 98.90 & 91.70 & 79.30 & 66.50 & 54.90 & 44.80 & 35.20 \\
\hline $9 \mathrm{H}$ & 161.40 & 4.911 & 100.00 & 98.71 & 95.32 & 83.58 & 69.65 & 57.61 & 46.47 & 35.62 \\
\hline $9 \mathrm{H}$ & 163.00 & 4.947 & 99.90 & 98.59 & 89.08 & 75.63 & 63.30 & 51.87 & 41.96 & 33.77 \\
\hline $9 \mathrm{H}$ & 165.92 & 5.013 & 99.40 & 97.90 & 88.32 & 71.56 & 57.88 & 47.30 & 39.32 & 31.84 \\
\hline $9 \mathrm{H}$ & 167.41 & 5.058 & 100.00 & 99.90 & 97.37 & 85.84 & 72.19 & 58.95 & 47.42 & 36.80 \\
\hline $9 \mathrm{H}$ & 168.19 & 5.079 & 99.90 & 98.80 & 89.62 & 74.95 & 61.38 & 49.80 & 39.62 & 30.94 \\
\hline $10 \mathrm{H}$ & 169.30 & 5.109 & 98.10 & 95.59 & 92.03 & 85.09 & 73.04 & 59.55 & 47.60 & 36.14 \\
\hline $10 \mathrm{H}$ & 170.80 & 5.149 & 99.90 & 99.20 & 91.16 & 77.19 & 64 & 47 & 43 & 33.87 \\
\hline $10 \mathrm{H}$ & 172.30 & 5.189 & 99.80 & 99.60 & 96.90 & 85.11 & 70.43 & 56.14 & 45.35 & 34.37 \\
\hline $10 \mathrm{H}$ & 173.20 & 5.213 & 100.00 & 99.10 & 97.70 & 89.30 & 75.30 & 60.70 & 48.30 & 37.20 \\
\hline $10 \mathrm{H}$ & 173.80 & 5.229 & 98.50 & 98.50 & 95.91 & 88.35 & 77.81 & 64.67 & 52.63 & 40.69 \\
\hline $10 \mathrm{H}$ & 175.30 & 5.270 & 100.00 & 99.29 & 87.39 & 71.95 & 61.55 & 52.17 & 44.80 & 37.03 \\
\hline $10 \mathrm{H}$ & 176.80 & 5.310 & 99.84 & 99.54 & 98.14 & 91.15 & 79 & 76 & 52.27 & 40.78 \\
\hline $10 \mathrm{H}$ & 177.63 & 5.332 & 100.00 & 98.68 & 89.57 & 75.30 & 63.06 & 50.91 & 41.09 & 30.67 \\
\hline $10 \mathrm{H}$ & 185.24 & 5.384 & 99.84 & 99.64 & 95.83 & 86.81 & 74.38 & 61.85 & 50.22 & 38.89 \\
\hline $12 \mathrm{H}$ & 188.31 & 5.448 & 99.70 & 98.09 & 86.94 & 68.74 & 56.99 & 47.04 & 39.40 & 31.46 \\
\hline $12 \mathrm{H}$ & 192.90 & 5.543 & 93.70 & 92.37 & 90.76 & 84.98 & 73.52 & 58.83 & 45.29 & 34.58 \\
\hline $13 \mathrm{H}$ & 198.13 & 565 & 93.70 & 93.51 & 90.39 & 78.40 & 65.93 & 54 & 44.58 & 35.42 \\
\hline $13 \mathrm{H}$ & 201.79 & 5.728 & 98.70 & 97.51 & 95.93 & 90.39 & 78.72 & 62.50 & 49.25 & 37.78 \\
\hline $14 X$ & 205.19 & 5.799 & 97.70 & 97.01 & 92.46 & 84.36 & 73.00 & 58.98 & 46.43 & 34.77 \\
\hline $14 X$ & 207.80 & 5.854 & 100.00 & 98.89 & 94.25 & 84.66 & 71.14 & 58.53 & 47.63 & 35.82 \\
\hline $14 X$ & 210.36 & 5.907 & 91.70 & 91.33 & 89.02 & 83.58 & 72.33 & 57.94 & 45.67 & 34.04 \\
\hline $15 X$ & 215.19 & 6.012 & 93.20 & 90.21 & 85.25 & 80.38 & 71.68 & 60.45 & 49.31 & 38.74 \\
\hline $15 X$ & 216.38 & 6.037 & 99.90 & 99.40 & 91.84 & 80.87 & 69.39 & 57.60 & 47.03 & 37.56 \\
\hline $15 x$ & 217.80 & 6.068 & 93.50 & 92.66 & 90.12 & 85.15 & 76.24 & 62.65 & 49.33 & 36.11 \\
\hline $16 \mathrm{X}$ & 226.73 & 6.178 & 100.00 & 99.90 & 97.38 & 88.62 & 75.63 & 62.13 & 50.35 & 38.27 \\
\hline $17 X$ & 236.00 & 6.244 & 99.90 & 99.39 & 98.59 & 90.50 & 77.25 & 63.30 & 51.47 & 39.74 \\
\hline $17 X$ & 236.21 & 6.246 & 99.90 & 99.09 & 96.06 & 83.13 & 68.38 & 54.34 & 43.94 & 32.73 \\
\hline $17 X$ & 236.40 & 6.247 & 97.40 & 97.30 & 93.26 & 86.07 & 71.89 & 55.84 & 44.32 & 32.50 \\
\hline $17 X$ & 236.60 & 6.248 & 93.60 & 91.34 & 82.13 & 71.70 & 59.96 & 47.65 & 37.40 & 29.54 \\
\hline $17 X$ & 236.80 & 6.250 & 98.40 & 98.40 & 96.18 & 91.44 & 79.93 & 65.50 & 52.88 & 40.87 \\
\hline $17 X$ & 237.01 & 6.251 & 96.80 & 95.20 & 83.20 & 67.30 & 54.00 & 42.50 & 34.50 & 23.90 \\
\hline $17 X$ & 237.20 & 6.253 & 99.90 & 98.26 & 89.66 & 78.61 & 67.04 & 55.27 & 46.47 & 35.62 \\
\hline $17 X$ & 237.40 & 6.254 & 99.90 & 98.16 & 80.15 & 60.39 & 48.82 & 40.02 & 32.34 & 24.67 \\
\hline $17 X$ & 238.00 & 6.258 & 99.80 & 99.80 & 98.76 & 93.16 & 80.30 & 66.19 & 54.15 & 41.39 \\
\hline $17 X$ & 238.58 & 6.263 & 99.80 & 97.60 & 88.22 & 72.65 & 58.78 & 46.01 & 36.03 & 25.35 \\
\hline $17 X$ & 239.09 & 6.266 & 99.90 & 99.90 & 95.96 & 86.16 & 73.64 & 58.79 & 46.06 & 34.04 \\
\hline $17 X$ & 239.30 & 6.268 & 97.10 & 95.81 & 90.35 & 82.90 & 70.89 & 57.58 & 46.76 & 36.24 \\
\hline $17 X$ & 239.69 & 6.274 & 88.70 & 85.28 & 75.30 & 65.60 & 56.36 & 47.40 & 39.64 & 31.51 \\
\hline $17 X$ & 240.10 & 6.280 & 99.40 & 97.89 & 94.27 & 84.83 & 72.06 & 59.50 & 48.04 & 36.68 \\
\hline $17 X$ & 240.75 & 6.291 & 99.90 & 99.90 & 95.33 & 80.49 & 67.07 & 53.96 & 42.07 & 30.79 \\
\hline $17 X$ & 242.54 & 6.320 & 97.60 & 95.34 & 93.18 & 85.52 & 73.05 & 58.42 & 47.33 & 35.74 \\
\hline
\end{tabular}


C.J. Pudsey

DATA Report: Grain-Size DATA

Table T2 (continued).

\begin{tabular}{|c|c|c|c|c|c|c|c|c|c|c|}
\hline \multirow[b]{2}{*}{ Core } & \multirow{2}{*}{$\begin{array}{l}\text { Depth } \\
\text { (mbsf) }\end{array}$} & \multirow{2}{*}{$\begin{array}{l}\text { Age } \\
(\mathrm{Ma})\end{array}$} & \multicolumn{8}{|c|}{ Grain size $(\phi)$} \\
\hline & & & 4 & 5 & 6 & 7 & 8 & 9 & 10 & 11 \\
\hline $18 \mathrm{X}$ & 244.03 & 6.344 & 98.30 & 90.88 & 75.03 & 64.40 & 55.17 & 45.24 & 36.51 & 27.99 \\
\hline $18 \mathrm{X}$ & 246.30 & 6.381 & 100.00 & 98.89 & 92.13 & 78.91 & 66.90 & 55.50 & 45.81 & 36.83 \\
\hline $18 \mathrm{X}$ & 248.64 & 6.419 & 100.00 & 99.60 & 96.39 & 87.45 & 75.70 & 64.26 & 53.21 & 41.97 \\
\hline $18 \mathrm{X}$ & 250.49 & 6.449 & 100.00 & 99.00 & 93.99 & 83.57 & 71.24 & 59.42 & 47.49 & 35.47 \\
\hline $18 \mathrm{X}$ & 252.33 & 6.479 & 75.80 & 71.42 & 64.58 & 57.66 & 48.28 & 38.98 & 31.06 & 23.75 \\
\hline $20 x$ & 264.91 & 6.643 & 88.40 & 81.78 & 71.04 & 63.35 & 54.94 & 45.45 & 37.13 & 28.27 \\
\hline $20 x$ & 268.10 & 6.678 & 99.90 & 98.49 & 93.86 & 81.97 & 68.68 & 56.40 & 45.32 & 34.24 \\
\hline $20 x$ & 269.44 & 6.692 & 86.50 & 78.50 & 71.20 & 62.77 & 54.06 & 44.74 & 36.04 & 27.87 \\
\hline $20 x$ & 271.44 & 6.714 & 99.80 & 99.80 & 97.17 & 88.69 & 75.66 & 61.92 & 49.09 & 36.57 \\
\hline $21 x$ & 274.88 & 6.751 & 99.90 & 99.50 & 97.89 & 91.36 & 80.20 & 67.84 & 56.08 & 43.72 \\
\hline $21 x$ & 276.67 & 6.770 & 96.00 & 84.07 & 68.73 & 58.75 & 50.44 & 41.84 & 33.92 & 27.18 \\
\hline $21 x$ & 281.14 & 6.818 & 100.00 & 99.50 & 94.55 & 82.85 & 69.83 & 56.81 & 45.31 & 35.02 \\
\hline $22 X$ & 284.74 & 6.857 & 100.00 & 98.39 & 96.49 & 85.24 & 71.29 & 58.33 & 46.99 & 36.04 \\
\hline $23 x$ & 292.70 & 6.944 & 100.00 & 99.70 & 96.18 & 86.65 & 74.10 & 62.85 & 52.31 & 42.07 \\
\hline $23 x$ & 297.23 & 7.003 & 99.90 & 99.90 & 97.57 & 87.44 & 73.96 & 59.37 & 47.82 & 36.58 \\
\hline $23 x$ & 300.25 & 7.042 & 100.00 & 99.70 & 98.09 & 90.64 & 76.26 & 61.37 & 48.29 & 35.41 \\
\hline
\end{tabular}

Notes: Fine fraction $=$ percentage finer than each 1- $\phi$ interval. Sand percentage can be obtained by subtracting the value in the $4-\phi$ column from 100 . 
C.J. Pudsey

DATA Report: Grain-Size DATA

Table T3. Median diameter, mode, sorting, and skewness, Site 1095. (Continued on next page.)

\begin{tabular}{|c|c|c|c|c|c|c|}
\hline Core & $\begin{array}{l}\text { Depth } \\
\text { (mbsf) }\end{array}$ & $\begin{array}{l}\text { Age } \\
(\mathrm{Ma})\end{array}$ & $\begin{array}{l}\text { Diameter } \\
\quad \phi 50\end{array}$ & Sorting & Skewness & $\begin{array}{c}\text { Mode } \\
(\phi)\end{array}$ \\
\hline \multicolumn{7}{|c|}{ 178-1095A- } \\
\hline $1 \mathrm{H}$ & 0.90 & 0.041 & 10.5 & 2.75 & 0.018 & 8 \\
\hline $1 \mathrm{H}$ & 1.66 & 0.076 & 9.8 & 2.5 & 0.040 & 8.1 \\
\hline $1 \mathrm{H}$ & 2.12 & 0.097 & 8.75 & 2.55 & 0.176 & 7.6 \\
\hline $1 \mathrm{H}$ & 2.59 & 0.118 & 8.85 & 3.4 & 0.015 & 7.9 \\
\hline $1 \mathrm{H}$ & 3.41 & 0.155 & 10.75 & 2.975 & 0.059 & \\
\hline $1 \mathrm{H}$ & 4.41 & 0.201 & 8.9 & 2.65 & 0.113 & 7.8 \\
\hline \multicolumn{7}{|c|}{ 178-1095D- } \\
\hline $1 \mathrm{H}$ & 4.59 & 0.209 & 8.9 & 2.85 & 0.140 & 7.9 \\
\hline $1 \mathrm{H}$ & 4.69 & 0.214 & 9.4 & 3.175 & 0.260 & 7.9 \\
\hline $1 \mathrm{H}$ & 4.79 & 0.218 & 9.5 & 3.15 & 0.143 & 8 \\
\hline $1 \mathrm{H}$ & 4.94 & 0.225 & 9.9 & 3.6 & 0.083 & 8.1 \\
\hline $1 \mathrm{H}$ & 5.09 & 0.232 & 9.6 & 3.3 & 0.106 & 7.9 \\
\hline $1 \mathrm{H}$ & 5.32 & 0.242 & 9.55 & 3.1 & 0.177 & 8 \\
\hline $1 \mathrm{H}$ & 5.49 & 0.250 & 9.2 & 3.075 & 0.220 & 7.9 \\
\hline $1 \mathrm{H}$ & 5.59 & 0.255 & 9.25 & 2.9 & 0.224 & 7.8 \\
\hline $1 \mathrm{H}$ & 5.69 & 0.259 & 9 & 2.75 & 0.200 & 7.8 \\
\hline $1 \mathrm{H}$ & 5.80 & 0.264 & 9.6 & 2.9 & 0.155 & 8 \\
\hline $1 \mathrm{H}$ & 5.90 & 0.269 & 9.7 & 2.95 & 0.153 & 8 \\
\hline $1 \mathrm{H}$ & 6.04 & 0.275 & 10.05 & 3.2 & 0.078 & 8 \\
\hline $1 \mathrm{H}$ & 6.34 & 0.289 & 10.3 & 2.925 & 0.060 & 8.1 \\
\hline $1 \mathrm{H}$ & 6.64 & 0.303 & 9.95 & 3.325 & 0.128 & 8.2 \\
\hline $1 \mathrm{H}$ & 6.94 & 0.316 & 9.55 & 4.175 & -0.090 & 7.9 \\
\hline $1 \mathrm{H}$ & 7.25 & 0.330 & 9.65 & 3.1 & 0.145 & 7.8 \\
\hline $1 \mathrm{H}$ & 7.54 & 0.344 & 9.35 & 3.075 & 0.187 & 7.8 \\
\hline $1 \mathrm{H}$ & 7.84 & 0.357 & 9.3 & 3.225 & 0.225 & 8 \\
\hline $1 \mathrm{H}$ & 8.19 & 0.373 & 10 & 3.325 & 0.128 & 8 \\
\hline \multicolumn{7}{|c|}{ 178-1095D- } \\
\hline $2 \mathrm{H}$ & 10.02 & 0.456 & 10.1 & 3.175 & 0.118 & 7.9 \\
\hline $2 \mathrm{H}$ & 10.39 & 0.473 & 10.35 & 3.2 & 0.078 & 8.1 \\
\hline $2 \mathrm{H}$ & 10.84 & 0.494 & 9.5 & 2.675 & 0.121 & 8.1 \\
\hline $2 \mathrm{H}$ & 11.89 & 0.542 & 9.6 & 3.3 & 0.152 & 7.9 \\
\hline $2 \mathrm{H}$ & 12.34 & 0.562 & 10.25 & 3.125 & 0.104 & 8.4 \\
\hline $2 \mathrm{H}$ & 12.92 & 0.589 & 10.45 & 3.2 & 0.078 & 7.9 \\
\hline $2 \mathrm{H}$ & 13.30 & 0.606 & 9.9 & 3.3 & 0.136 & 8.2 \\
\hline $2 \mathrm{H}$ & 13.84 & 0.631 & 10.2 & 3.225 & 0.116 & 8 \\
\hline $2 \mathrm{H}$ & 14.39 & 0.656 & 10.3 & 3.25 & 0.092 & 8.1 \\
\hline \multicolumn{7}{|c|}{ 178-1095A- } \\
\hline $3 \mathrm{H}$ & 15.09 & 0.687 & 9.65 & 3.725 & 0.047 & 7.9 \\
\hline $3 \mathrm{H}$ & 15.11 & 0.688 & 10.2 & 3.275 & 0.099 & \\
\hline $3 \mathrm{H}$ & 15.56 & 0.709 & 9.3 & 2.45 & 0.184 & 8 \\
\hline $3 \mathrm{H}$ & 16.52 & 0.753 & 10.35 & 3.025 & 0.157 & 8.1 \\
\hline $3 \mathrm{H}$ & 17.67 & 0.802 & 9.6 & 2.55 & 0.098 & 8 \\
\hline $3 \mathrm{H}$ & 19.33 & 0.859 & 10.15 & 3.375 & 0.081 & \\
\hline $4 \mathrm{H}$ & 22.49 & 0.968 & 10.4 & 3.425 & 0.109 & 7.9 \\
\hline $4 \mathrm{H}$ & 24.01 & 1.000 & 9.6 & 3.725 & 0.101 & \\
\hline $4 \mathrm{H}$ & 25.00 & 1.012 & 11 & 3.1 & 0.032 & 8.4 \\
\hline $4 \mathrm{H}$ & 25.95 & 1.024 & 9.2 & 2.55 & 0.157 & 8 \\
\hline $4 \mathrm{H}$ & 26.99 & 1.036 & 9.1 & 3.825 & 0.085 & 8 \\
\hline $4 \mathrm{H}$ & 27.76 & 1.046 & 8.22 & 2.725 & 0.277 & 7.9 \\
\hline $4 \mathrm{H}$ & 28.54 & 1.055 & 9.3 & 2.95 & 0.119 & 7.9 \\
\hline $5 \mathrm{H}$ & 31.10 & 1.101 & 9 & 2.2 & 0.205 & 7.9 \\
\hline $5 \mathrm{H}$ & 31.50 & 1.110 & 8.35 & 2.775 & 0.207 & 7.9 \\
\hline $5 \mathrm{H}$ & 31.90 & 1.119 & 9.1 & 3 & 0.067 & 8.3 \\
\hline $5 \mathrm{H}$ & 32.30 & 1.128 & 9 & 2.8 & 0.214 & 7.9 \\
\hline $5 \mathrm{H}$ & 32.80 & 1.140 & 10.3 & 3.45 & 0.101 & \\
\hline $5 \mathrm{H}$ & 33.10 & 1.147 & 10.1 & 3.275 & 0.084 & \\
\hline $5 \mathrm{H}$ & 33.53 & 1.156 & 8.9 & 3.025 & 0.223 & 7.5 \\
\hline $5 \mathrm{H}$ & 33.88 & 1.164 & 10 & 2.95 & 0.051 & \\
\hline $5 \mathrm{H}$ & 34.27 & 1.173 & 10.45 & 3.05 & 0.098 & 8.2 \\
\hline $5 \mathrm{H}$ & 34.70 & 1.183 & 10.25 & 3.05 & 0.082 & 8.2 \\
\hline $5 \mathrm{H}$ & 35.00 & 1.190 & 10.5 & 3.2 & 0.031 & 8.2 \\
\hline $5 \mathrm{H}$ & 35.39 & 1.199 & 9.95 & 3.05 & 0.033 & 8.4 \\
\hline $5 \mathrm{H}$ & 35.80 & 1.208 & 9.9 & 3 & 0.100 & 8 \\
\hline $5 \mathrm{H}$ & 36.09 & 1.215 & 8.1 & 2.625 & 0.219 & 7.5 \\
\hline $5 \mathrm{H}$ & 36.34 & 1.221 & 9 & 3.35 & 0.194 & 6.3 \\
\hline $6 \mathrm{H}$ & 40.76 & 1.322 & 10.45 & 3.35 & 0.000 & 8.1 \\
\hline $6 \mathrm{H}$ & 41.80 & 1.345 & 9.4 & 4.1 & -0.049 & 8.3 \\
\hline
\end{tabular}

\begin{tabular}{|c|c|c|c|c|c|c|}
\hline Core & $\begin{array}{l}\text { Depth } \\
\text { (mbsf) }\end{array}$ & $\begin{array}{l}\text { Age } \\
(\mathrm{Ma})\end{array}$ & $\begin{array}{c}\text { Diameter } \\
\phi 50\end{array}$ & Sorting & Skewness & $\begin{array}{c}\text { Mode } \\
(\phi)\end{array}$ \\
\hline $6 \mathrm{H}$ & 43.69 & 1.388 & 9.15 & 3.725 & 0.101 & 8.3 \\
\hline $6 \mathrm{H}$ & 44.91 & 1.416 & 10.45 & 3 & 0.067 & 7.9 \\
\hline $6 \mathrm{H}$ & 46.78 & 1.459 & 9.6 & 3.425 & -0.080 & 8.1 \\
\hline $6 \mathrm{H}$ & 47.95 & 1.486 & 10.5 & 2.95 & -0.017 & 8 \\
\hline $7 \mathrm{H}$ & 50.06 & 1.534 & 8.5 & 3.225 & 0.225 & 6.4 \\
\hline $7 \mathrm{H}$ & 51.39 & 1.564 & 7.75 & 3.625 & 0.352 & 5.5 \\
\hline $7 \mathrm{H}$ & 51.90 & 1.576 & 10.1 & 2.975 & 0.059 & 8 \\
\hline $7 \mathrm{H}$ & 52.89 & 1.599 & 9.2 & 3.225 & 0.023 & 8.1 \\
\hline \multicolumn{7}{|c|}{ 178-1095D- } \\
\hline $6 \mathrm{H}$ & 53.41 & 1.599 & 10.3 & 2.95 & 0.085 & 8.1 \\
\hline $6 \mathrm{H}$ & 53.67 & 1.599 & 10.3 & 3.35 & 0.075 & 8.3 \\
\hline $6 \mathrm{H}$ & 53.86 & 1.599 & 9.9 & 4.05 & -0.160 & 8.4 \\
\hline $6 \mathrm{H}$ & 54.07 & 1.599 & 9.95 & 3.8 & 0.026 & 8.2 \\
\hline $6 \mathrm{H}$ & 54.26 & 1.599 & 10.8 & 3.5 & 0.000 & 8.1 \\
\hline \multicolumn{7}{|c|}{$178-1095 \mathrm{~A}-$} \\
\hline $7 \mathrm{H}$ & 54.39 & 1.633 & 9.5 & 3.3 & 0.121 & \\
\hline \multicolumn{7}{|c|}{ 178-1095D- } \\
\hline $6 \mathrm{H}$ & 54.46 & 1.635 & 10.9 & 3.25 & 0.031 & 8.1 \\
\hline $6 \mathrm{H}$ & 54.67 & 1.639 & 10.3 & 3.125 & 0.072 & 8.2 \\
\hline $6 \mathrm{H}$ & 54.86 & 1.644 & 10.85 & 3.1 & 0.048 & 8.1 \\
\hline $6 \mathrm{H}$ & 55.06 & 1.648 & 10.4 & 2.925 & 0.094 & 8.1 \\
\hline $6 \mathrm{H}$ & 55.33 & 1.654 & 9.95 & 3.225 & 0.116 & 7.8 \\
\hline $6 \mathrm{H}$ & 55.89 & 1.667 & 10.25 & 2.975 & 0.092 & 8.3 \\
\hline $6 \mathrm{H}$ & 57.39 & 1.701 & 7.45 & 3.375 & 0.407 & 6.2 \\
\hline $6 \mathrm{H}$ & 58.79 & 1.733 & 9.6 & 4.25 & -0.224 & \\
\hline $8 \mathrm{H}$ & 60.20 & 1.766 & 9.55 & 3.3 & 0.015 & \\
\hline $8 \mathrm{H}$ & 61.70 & 1.842 & 9 & 3.275 & 0.191 & 6.3 \\
\hline $8 \mathrm{H}$ & 63.20 & 1.925 & 9.4 & 3.6 & -0.014 & \\
\hline $8 \mathrm{H}$ & 64.10 & 1.975 & 8.95 & 3.575 & 0.133 & 5.8 \\
\hline $8 \mathrm{H}$ & 65.60 & 2.058 & 8.65 & 3.025 & 0.025 & 8 \\
\hline $8 \mathrm{H}$ & 67.10 & 2.140 & 9.05 & 4.2 & 0.083 & 4.9 \\
\hline $8 \mathrm{H}$ & 68.00 & 2.190 & 9.7 & 3.35 & 0.134 & 7.9 \\
\hline $9 \mathrm{H}$ & 69.31 & 2.262 & 8.65 & 3 & 0.233 & 6.7 \\
\hline $9 \mathrm{H}$ & 71.00 & 2.356 & 9.45 & 3.275 & 0.130 & \\
\hline $9 \mathrm{H}$ & 71.87 & 2.404 & 7.7 & 5.9 & -0.305 & 7.8 \\
\hline $9 \mathrm{H}$ & 73.27 & 2.481 & 9.3 & 3.275 & 0.160 & 6.5 \\
\hline $9 \mathrm{H}$ & 74.81 & 2.566 & 10.15 & 3.35 & 0.015 & \\
\hline $9 \mathrm{H}$ & 77.02 & 2.688 & 9.8 & 3.375 & 0.007 & \\
\hline $10 \mathrm{H}$ & 78.77 & 2.784 & 7.6 & 3.25 & 0.462 & 6 \\
\hline $10 \mathrm{H}$ & 79.02 & 2.798 & 10.2 & 2.9 & 0.069 & 8.1 \\
\hline $10 \mathrm{H}$ & 79.14 & 2.805 & 8.95 & 4.7 & -0.266 & 8.2 \\
\hline $10 \mathrm{H}$ & 79.27 & 2.812 & 8.5 & 2.675 & 0.103 & 7.8 \\
\hline $10 \mathrm{H}$ & 79.44 & 2.821 & 9.1 & 2.775 & 0.117 & 8.3 \\
\hline $10 \mathrm{H}$ & 79.59 & 2.830 & 9.55 & 3.4 & -0.015 & 9 \\
\hline $10 \mathrm{H}$ & 79.81 & 2.842 & 8.3 & 3.225 & 0.240 & 5.9 \\
\hline $10 \mathrm{H}$ & 80.07 & 2.856 & 9.75 & 3.025 & 0.174 & \\
\hline $10 \mathrm{H}$ & 80.45 & 2.877 & 9.6 & 3.075 & 0.106 & \\
\hline $10 \mathrm{H}$ & 80.83 & 2.898 & 10.1 & 3.275 & 0.130 & 8.5 \\
\hline $10 \mathrm{H}$ & 81.19 & 2.918 & 9 & 2.775 & 0.171 & 8.4 \\
\hline $10 \mathrm{H}$ & 81.34 & 2.926 & 10.45 & 3.15 & 0.032 & \\
\hline $10 \mathrm{H}$ & 81.47 & 2.933 & 8.35 & 2.9 & 0.000 & 8 \\
\hline $10 \mathrm{H}$ & 81.65 & 2.943 & 9 & 2.85 & 0.158 & 8.1 \\
\hline $10 \mathrm{H}$ & 81.81 & 2.952 & 9.35 & 3.125 & 0.152 & 8 \\
\hline $10 \mathrm{H}$ & 82.05 & 2.965 & 9.9 & 2.95 & 0.068 & 8.3 \\
\hline $10 \mathrm{H}$ & 82.82 & 3.008 & 10.2 & 3.025 & 0.058 & \\
\hline $10 \mathrm{H}$ & 84.67 & 3.079 & 9.55 & 3.275 & 0.115 & 6.6 \\
\hline $10 \mathrm{H}$ & 87.04 & 3.152 & 9.2 & 2.725 & 0.119 & 8 \\
\hline $10 \mathrm{H}$ & 88.85 & 3.207 & 10.4 & 2.85 & 0.000 & \\
\hline $10 \mathrm{H}$ & 90.24 & 3.250 & 10.05 & 3.025 & 0.124 & 7.8 \\
\hline $10 \mathrm{H}$ & 91.92 & 3.302 & 9.55 & 2.975 & 0.109 & 7.9 \\
\hline \multicolumn{7}{|c|}{ 178-1095B- } \\
\hline $2 \mathrm{H}$ & 94.20 & 3.372 & 9.8 & 3.2 & 0.109 & 8 \\
\hline $2 \mathrm{H}$ & 95.36 & 3.407 & 9.5 & 2.75 & 0.127 & 8.2 \\
\hline $2 \mathrm{H}$ & 96.75 & 3.450 & 8.3 & 3.45 & 0.275 & 6 \\
\hline $2 \mathrm{H}$ & 98.19 & 3.494 & 10.4 & 3.25 & 0.046 & 8.1 \\
\hline $2 \mathrm{H}$ & 99.87 & 3.546 & 10.45 & 3.075 & 0.024 & 8.6 \\
\hline $3 \mathrm{H}$ & 104.09 & 3.654 & 9.55 & 3.25 & 0.062 & 7.8 \\
\hline
\end{tabular}


C.J. Pudsey

DATA Report: Grain-Size DATA

Table T3 (continued).

\begin{tabular}{|c|c|c|c|c|c|c|}
\hline Core & $\begin{array}{l}\text { Depth } \\
\text { (mbsf) }\end{array}$ & $\begin{array}{l}\text { Age } \\
(\mathrm{Ma})\end{array}$ & $\begin{array}{l}\text { Diameter } \\
\phi 50\end{array}$ & Sorting & Skewness & $\begin{array}{c}\text { Mode } \\
(\phi)\end{array}$ \\
\hline $3 \mathrm{H}$ & 107.72 & 3.740 & 9.55 & 3.075 & 0.106 & \\
\hline $3 \mathrm{H}$ & 109.23 & 3.776 & 9.7 & 3.15 & 0.016 & \\
\hline $3 \mathrm{H}$ & 111.40 & 3.828 & 9.7 & 3.125 & 0.120 & 7.9 \\
\hline $4 \mathrm{H}$ & 114.28 & 3.896 & 9.6 & 3.15 & 0.095 & \\
\hline $4 \mathrm{H}$ & 116.51 & 3.949 & 8.35 & 3.15 & 0.286 & 6.1 \\
\hline $4 \mathrm{H}$ & 118.34 & 3.993 & 10.15 & 2.725 & 0.009 & 8.3 \\
\hline $4 \mathrm{H}$ & 120.24 & 4.038 & 10.3 & 2.625 & 0.067 & 8.2 \\
\hline $5 \mathrm{H}$ & 122.08 & 4.082 & 9.2 & 3.05 & 0.115 & \\
\hline $5 \mathrm{H}$ & 126.44 & 4.183 & 9.8 & 2.5 & 0.040 & \\
\hline $6 \mathrm{H}$ & 133.38 & 4.270 & 10.15 & 2.95 & 0.068 & 7.8 \\
\hline $6 \mathrm{H}$ & 135.17 & 4.296 & 9.3 & 2.725 & 0.101 & 8.2 \\
\hline $6 \mathrm{H}$ & 138.95 & 4.415 & 9.5 & 3.1 & 0.113 & \\
\hline $7 \mathrm{H}$ & 142.87 & 4.515 & 9.2 & 3.35 & 0.194 & 6.6 \\
\hline $7 \mathrm{H}$ & 144.22 & 4.540 & 10 & 2.9 & 0.069 & 7.9 \\
\hline $7 \mathrm{H}$ & 145.38 & 4.562 & 6.85 & 2.4 & 0.563 & 6.1 \\
\hline $7 \mathrm{H}$ & 146.67 & 4.586 & 8.95 & 3.1 & 0.177 & \\
\hline $7 \mathrm{H}$ & 148.16 & 4.614 & 9.95 & 2.85 & 0.105 & \\
\hline $7 \mathrm{H}$ & 149.07 & 4.633 & 8.5 & 3.175 & 0.260 & 6.4 \\
\hline $8 \mathrm{H}$ & 150.60 & 4.668 & 9.75 & 2.525 & 0.149 & \\
\hline $8 \mathrm{H}$ & 152.10 & 4.701 & 9.25 & 3.15 & -0.016 & \\
\hline $8 \mathrm{H}$ & 153.59 & 4.735 & 4.4 & 2.775 & & 4.9 \\
\hline $8 \mathrm{H}$ & 155.09 & 4.769 & 6.1 & 2.825 & 0.664 & 5.4 \\
\hline $8 \mathrm{H}$ & 156.60 & 4.803 & 9.4 & 3.4 & 0.059 & \\
\hline $8 \mathrm{H}$ & 158.10 & 4.836 & 10 & 3 & 0.067 & \\
\hline $8 \mathrm{H}$ & 158.69 & 4.850 & 10.05 & 2.475 & 0.071 & \\
\hline $9 \mathrm{H}$ & 159.90 & 4.877 & 9.5 & 3.15 & 0.095 & \\
\hline $9 \mathrm{H}$ & 161.40 & 4.911 & 9.7 & 2.9 & 0.069 & \\
\hline $9 \mathrm{H}$ & 163.00 & 4.947 & 9.2 & 3.475 & 0.180 & 6.1 \\
\hline $9 \mathrm{H}$ & 165.92 & 5.013 & 8.7 & 3.475 & 0.295 & 6.4 \\
\hline $9 \mathrm{H}$ & 167.41 & 5.058 & 9.8 & 2.85 & 0.088 & \\
\hline $9 \mathrm{H}$ & 168.19 & 5.079 & 9 & 3.2 & 0.188 & 6.4 \\
\hline $10 \mathrm{H}$ & 169.30 & 5.109 & 9.8 & 2.825 & 0.044 & \\
\hline $10 \mathrm{H}$ & 170.80 & 5.149 & 9.3 & 3.175 & 0.134 & \\
\hline $10 \mathrm{H}$ & 172.30 & 5.189 & 9.55 & 2.825 & 0.133 & \\
\hline $10 \mathrm{H}$ & 173.20 & 5.213 & 9.85 & 2.75 & 0.109 & 8.1 \\
\hline $10 \mathrm{H}$ & 173.80 & 5.229 & 10.2 & 2.8 & 0.036 & \\
\hline $10 \mathrm{H}$ & 175.30 & 5.270 & 9.3 & 3.7 & 0.162 & 6.4 \\
\hline $10 \mathrm{H}$ & 176.80 & 5.310 & 10.2 & 2.75 & 0.055 & 8.4 \\
\hline $10 \mathrm{H}$ & 177.63 & 5.332 & 9.1 & 3.05 & 0.098 & 6.1 \\
\hline $11 \mathrm{H}$ & 179.27 & 5.259 & 10.55 & 2.5 & 0.060 & \\
\hline $11 \mathrm{H}$ & 185.24 & 5.384 & 10 & 2.9 & 0.052 & \\
\hline $12 \mathrm{H}$ & 188.31 & 5.448 & 8.7 & 3.375 & 0.244 & 6.1 \\
\hline
\end{tabular}

\begin{tabular}{|c|c|c|c|c|c|c|}
\hline Core & $\begin{array}{l}\text { Depth } \\
\text { (mbsf) }\end{array}$ & $\begin{array}{l}\text { Age } \\
(\mathrm{Ma})\end{array}$ & $\begin{array}{c}\text { Diameter } \\
\phi 50\end{array}$ & Sorting & Skewness & $\begin{array}{c}\text { Mode } \\
(\phi)\end{array}$ \\
\hline $12 \mathrm{H}$ & 192.90 & 5.543 & 9.65 & 2.75 & 0.109 & \\
\hline $13 \mathrm{H}$ & 198.13 & 5.652 & 9.45 & 3.2 & 0.109 & \\
\hline $13 \mathrm{H}$ & 201.79 & 5.728 & 9.9 & 2.625 & 0.143 & 8.3 \\
\hline $14 X$ & 205.19 & 5.799 & 9.7 & 2.8 & 0.054 & 8.2 \\
\hline $14 X$ & 207.80 & 5.854 & 9.8 & 2.8 & 0.036 & \\
\hline $14 X$ & 210.36 & 5.907 & 9.6 & 2.8 & 0.071 & 8.5 \\
\hline $15 X$ & 215.19 & 6.012 & 9.95 & 3.425 & -0.066 & \\
\hline $15 X$ & 216.38 & 6.037 & 9.7 & 3.275 & 0.084 & \\
\hline $15 X$ & 217.80 & 6.068 & 9.95 & 2.65 & -0.038 & \\
\hline $16 X$ & 226.73 & 6.178 & 10 & 2.7 & 0.037 & \\
\hline $17 X$ & 236.00 & 6.244 & 10.15 & 2.75 & 0.055 & \\
\hline $17 X$ & 236.21 & 6.246 & 9.4 & 2.775 & 0.117 & \\
\hline $17 X$ & 236.40 & 6.247 & 9.5 & 2.6 & 0.115 & 8.3 \\
\hline $17 X$ & 236.60 & 6.248 & 8.8 & 3.075 & 0.041 & \\
\hline $17 X$ & 236.80 & 6.250 & 10.25 & 2.675 & 0.065 & \\
\hline $17 X$ & 237.01 & 6.251 & 9.3 & 2.925 & -0.145 & 6.1 \\
\hline $17 X$ & 237.20 & 6.253 & 9.55 & 3.2 & 0.047 & \\
\hline $17 X$ & 237.40 & 6.254 & 7.9 & 3.15 & 0.349 & 6 \\
\hline $17 X$ & 238.00 & 6.258 & 10.3 & 2.65 & 0.038 & 8.1 \\
\hline $17 X$ & 238.58 & 6.263 & 8.65 & 2.825 & 0.150 & 6.3 \\
\hline $17 X$ & 239.09 & 6.266 & 9.65 & 2.65 & 0.075 & \\
\hline $17 X$ & 239.30 & 6.268 & 9.7 & 2.975 & 0.059 & 8.3 \\
\hline $17 X$ & 239.69 & 6.274 & 8.7 & 3.95 & 0.089 & \\
\hline $17 X$ & 240.10 & 6.280 & 9.8 & 2.85 & 0.053 & \\
\hline $17 X$ & 240.75 & 6.291 & 9.35 & 2.775 & 0.063 & \\
\hline $17 X$ & 242.54 & 6.320 & 9.7 & 2.75 & 0.091 & 8.4 \\
\hline $18 X$ & 244.03 & 6.344 & 8.55 & 3.475 & 0.094 & 5.3 \\
\hline $18 X$ & 246.30 & 6.381 & 9.55 & 3.325 & 0.128 & \\
\hline $18 X$ & 248.64 & 6.419 & 10.3 & 3 & 0.033 & \\
\hline $18 X$ & 250.49 & 6.449 & 9.75 & 2.825 & 0.027 & \\
\hline $18 X$ & 252.33 & 6.479 & 7.8 & 6.05 & -0.289 & \\
\hline $20 x$ & 264.91 & 6.643 & 8.5 & 3.825 & 0.020 & \\
\hline $20 x$ & 268.10 & 6.678 & 9.6 & 2.875 & 0.043 & \\
\hline $20 x$ & 269.44 & 6.692 & 8.45 & 4 & -0.012 & \\
\hline $20 x$ & 271.44 & 6.714 & 9.8 & 2.6 & 0.077 & \\
\hline $21 x$ & 274.88 & 6.751 & 10.5 & 2.8 & 0.000 & \\
\hline $21 x$ & 276.67 & 6.770 & 8.05 & 3.725 & 0.181 & 5.1 \\
\hline $21 x$ & 281.14 & 6.818 & 9.6 & 2.925 & 0.077 & \\
\hline $22 x$ & 284.74 & 6.857 & 9.7 & 2.85 & 0.088 & \\
\hline $23 X$ & 292.70 & 6.944 & 10.25 & 3.15 & 0.048 & \\
\hline $23 x$ & 297.23 & 7.003 & 9.8 & 2.725 & 0.083 & 8.2 \\
\hline $23 x$ & 300.25 & 7.042 & 9.85 & 2.475 & 0.051 & 8 \\
\hline
\end{tabular}


C.J. Pudsey

DATA Report: Grain-Size DATA

Table T4. Diatom percentage, Site 1096.

\begin{tabular}{|c|c|c|c|}
\hline Hole & $\begin{array}{l}\text { Depth } \\
\text { (mbsf) }\end{array}$ & $\begin{array}{l}\text { Age } \\
(\mathrm{Ma})\end{array}$ & $\begin{array}{c}\text { Diatoms } \\
(\%)\end{array}$ \\
\hline \multicolumn{4}{|c|}{ 178-1096A- } \\
\hline & 0.00 & 0.000 & \\
\hline & 7.89 & 0.113 & 1 \\
\hline & 8.30 & 0.119 & 0.5 \\
\hline & 8.80 & 0.126 & 0.5 \\
\hline & 9.29 & 0.133 & 0.5 \\
\hline & 9.79 & 0.140 & 0.5 \\
\hline & 10.31 & 0.147 & 0.5 \\
\hline & 10.80 & 0.154 & 1 \\
\hline & 11.29 & 0.161 & 0.5 \\
\hline & 11.80 & 0.169 & 0.5 \\
\hline & 12.29 & 0.176 & 0.5 \\
\hline & 12.79 & 0.183 & 4 \\
\hline & 13.29 & 0.190 & 7 \\
\hline & 13.53 & 0.193 & 5 \\
\hline & 13.95 & 0.199 & 3 \\
\hline & 14.49 & 0.207 & 2 \\
\hline & 15.00 & 0.214 & 5 \\
\hline & 15.49 & 0.221 & 2 \\
\hline & 15.90 & 0.227 & 14 \\
\hline & 16.46 & 0.235 & 3 \\
\hline & 18.50 & 0.264 & 0.5 \\
\hline & 19.00 & 0.271 & 2 \\
\hline & 19.50 & 0.279 & 2 \\
\hline & 20.00 & 0.286 & 4 \\
\hline & 20.26 & 0.289 & 6 \\
\hline & 20.40 & 0.291 & 10 \\
\hline & 20.60 & 0.294 & 6 \\
\hline & 21.06 & 0.301 & 3 \\
\hline & 21.50 & 0.307 & 4 \\
\hline & 24.50 & 0.350 & 4 \\
\hline & 28.10 & 0.401 & 1 \\
\hline & 28.70 & 0.410 & 1 \\
\hline & 30.21 & 0.432 & 3 \\
\hline & 31.70 & 0.453 & 9 \\
\hline & 36.78 & 0.525 & 4.5 \\
\hline & 38.30 & 0.547 & 4 \\
\hline & 39.80 & 0.569 & 4 \\
\hline & 41.30 & 0.590 & 3 \\
\hline & 42.80 & 0.611 & 1 \\
\hline & 44.45 & 0.635 & 1 \\
\hline & 45.30 & 0.647 & 0.5 \\
\hline & 46.69 & 0.667 & 1 \\
\hline & 47.58 & 0.680 & 0 \\
\hline & 49.09 & 0.701 & 0 \\
\hline & 50.59 & 0.723 & 0.5 \\
\hline & 52.10 & 0.744 & 0 \\
\hline & 53.60 & 0.766 & 0.5 \\
\hline & 55.34 & 0.787 & 2 \\
\hline & 55.93 & 0.794 & 2 \\
\hline & 56.84 & 0.804 & 4 \\
\hline & 58.34 & 0.821 & 3 \\
\hline & 59.84 & 0.838 & 2 \\
\hline & 61.31 & 0.854 & 3 \\
\hline & 62.34 & 0.866 & 2 \\
\hline & 63.34 & 0.877 & 3 \\
\hline & 65.10 & 0.897 & 0.5 \\
\hline & 66.59 & 0.914 & 1 \\
\hline & 68.09 & 0.931 & 1 \\
\hline & 69.59 & 0.947 & 1 \\
\hline & 71.09 & 0.964 & 0 \\
\hline & 72.64 & 0.982 & 0 \\
\hline & 73.78 & 0.994 & 0 \\
\hline & 76.10 & 1.021 & 0 \\
\hline
\end{tabular}

\begin{tabular}{rrcc}
\hline Hole & $\begin{array}{r}\text { Depth } \\
(\mathrm{mbsf})\end{array}$ & $\begin{array}{c}\text { Age } \\
(\mathrm{Ma})\end{array}$ & $\begin{array}{c}\text { Diatoms } \\
(\%)\end{array}$ \\
\hline 77.60 & 1.037 & 0 \\
79.88 & 1.063 & 0 \\
80.27 & 1.067 & 0 \\
80.58 & 1.071 & 0 \\
80.87 & 1.074 & 0 \\
81.16 & 1.077 & 0 \\
81.45 & 1.081 & 1 \\
81.83 & 1.085 & 3 \\
82.05 & 1.087 & 1 \\
83.13 & 1.100 & 0 \\
87.09 & 1.144 & 0 \\
94.49 & 1.227 & 4 \\
97.39 & 1.260 & 4 \\
98.88 & 1.276 & 2 \\
100.40 & 1.294 & 1 \\
122.80 & 1.545 & 0 \\
123.56 & 1.554 & 0 \\
125.72 & 1.578 & 0 \\
128.70 & 1.612 & 0 \\
132.02 & 1.649 & 9 \\
134.23 & 1.674 & 13 \\
134.75 & 1.680 & 4.5 \\
137.75 & 1.713 & 0 \\
& &
\end{tabular}

\begin{tabular}{cccc}
\hline Hole & $\begin{array}{c}\text { Depth } \\
(\mathrm{mbsf})\end{array}$ & $\begin{array}{c}\text { Age } \\
(\mathrm{Ma})\end{array}$ & $\begin{array}{c}\text { Diatoms } \\
(\%)\end{array}$ \\
\hline 111.15 & 1.414 & 2 \\
111.56 & 1.419 & 0 \\
112.08 & 1.425 & 0 \\
112.49 & 1.429 & 0 \\
112.89 & 1.434 & 0 \\
113.29 & 1.438 & 0 \\
113.69 & 1.443 & 0 \\
115.30 & 1.461 & 0 \\
136.50 & 1.699 & 2 \\
137.20 & 1.707 & 0 \\
137.30 & 1.708 & 0 \\
137.40 & 1.709 & 0 \\
137.50 & 1.710 & 0 \\
137.60 & 1.712 & 0 \\
137.70 & 1.713 & 0 \\
138.00 & 1.716 & 2.5 \\
139.50 & 1.733 & 0.5 \\
141.00 & 1.750 & 0 \\
142.50 & 1.767 & 0.5 \\
143.40 & 1.777 & 0 \\
144.60 & 1.790 & 3.5
\end{tabular}

178-1096B-

$\begin{array}{lll}71.14 & 0.965 & 0\end{array}$

$\begin{array}{lll}74.14 & 0.999 & 0\end{array}$

$77.14 \quad 1.032$

$\begin{array}{lll}77.86 & 1.040 & 0\end{array}$

$78.05 \quad 1.042$

$78.26 \quad 1.045$

$78.45 \quad 1.047$

$\begin{array}{ll}78.65 & 1.049 \\ 78.84 & 1.051\end{array}$

$79.04 \quad 1.054$

$\begin{array}{lll}80.59 & 1.071 & 3\end{array}$

$81.10 \quad 1.077$

$81.50 \quad 1.081$

$81.90 \quad 1.086$

$82.29 \quad 1.090$

$82.69 \quad 1.095$

$\begin{array}{ll}83.19 & 1.100 \\ 83.79 & 1.107\end{array}$

$84.39 \quad 1.114$

$84.89 \quad 1.119$

$85.09 \quad 1.122$

$85.30 \quad 1.124$

$85.69 \quad 1.128$

$86.09 \quad 1.133$

$86.56 \quad 1.138$

$87.00 \quad 1.143$

$91.00 \quad 1.188$

$94.00 \quad 1.222$

$96.90 \quad 1.254$

$99.64 \quad 1.285$

$101.74 \quad 1.309$

$104.75 \quad 1.342$

$107.75 \quad 1.376$

$108.02 \quad 1.379$

$108.74 \quad 1.387$

$109.14 \quad 1.392$

$109.59 \quad 1.397$

$110.00 \quad 1.401$

$110.39 \quad 1.406$

$\begin{array}{lll}144.60 & 1.790 \quad 3.5\end{array}$

$\begin{array}{lll}146.10 & 1.807 & 5\end{array}$

$147.60 \quad 1.824 \quad 5$

$\begin{array}{lll}149.10 & 1.841 & 3\end{array}$

$\begin{array}{lll}150.26 & 1.854 & 2\end{array}$

$\begin{array}{lll}150.45 & 1.856 & 0.5\end{array}$

$\begin{array}{lll}151.79 & 1.871 & 0.5\end{array}$

$\begin{array}{lll}153.29 & 1.888 & 0.5\end{array}$

$154.48 \quad 1.901 \quad 3$

$156.29 \quad 1.922 \quad 0$

$156.99 \quad 1.929$

$\begin{array}{lll}158.09 & 1.942 & 1\end{array}$

$159.59 \quad 1.959$

$\begin{array}{lll}161.09 & 1.975 & 13.5\end{array}$

$\begin{array}{lll}162.59 & 1.992 & 16\end{array}$

$164.09 \quad 2.009 \quad 4$

$165.59 \quad 2.026 \quad 4$

$\begin{array}{lll}167.29 & 2.045 \quad 2\end{array}$

$\begin{array}{lll}174.70 & 2.128 & 2\end{array}$

$176.24 \quad 2.146 \quad 2$

$\begin{array}{lll}177.64 & 2.161 & 2\end{array}$

$179.15 \quad 2.178 \quad 4$

$\begin{array}{lll}184.09 & 2.234 & 8\end{array}$

$\begin{array}{lll}185.50 & 2.250 \quad 6\end{array}$

$\begin{array}{lll}187.00 & 2.267 \quad 6\end{array}$

$\begin{array}{lll}188.50 & 2.283 & 11\end{array}$

$190.00 \quad 2.300 \quad 9$

$\begin{array}{lll}191.50 & 2.317 \quad 9\end{array}$

$\begin{array}{lll}192.42 & 2.327 & 6\end{array}$

$203.19 \quad 2.449 \quad 4$

$204.69 \quad 2.465 \quad 4$

$206.19 \quad 2.482 \quad 5$

$207.69 \quad 2.499 \quad 6$

$209.19 \quad 2.516 \quad 4$

$210.69 \quad 2.533 \quad 15$

$211.94 \quad 2.547 \quad 16$

$213.70 \quad 2.567 \quad 5$

$215.19 \quad 2.583$

$\begin{array}{lll}216.69 & 2.585 & 19\end{array}$

$217.59 \quad 2.590 \quad 9$

$223.29 \quad 2.618 \quad 12$

$226.05 \quad 2.632 \quad 20$

$232.29 \quad 2.663 \quad 9$

$233.79 \quad 2.670 \quad 12$ 
C.J. Pudsey

DATA Report: Grain-Size DATA

Table T5. Fine-fraction $(>4 \phi)$ size distribution (percentage finer than each 1- $\phi$ interval), Site 1096. (Continued on next two pages.)

\begin{tabular}{|c|c|c|c|c|c|c|c|c|c|c|}
\hline \multirow[b]{2}{*}{ Core } & \multirow{2}{*}{$\begin{array}{l}\text { Depth } \\
\text { (mbsf) }\end{array}$} & \multirow{2}{*}{$\begin{array}{l}\text { Age } \\
\text { (Ma) }\end{array}$} & \multicolumn{8}{|c|}{ Grain size $(\phi)$} \\
\hline & & & 4 & 5 & 6 & 7 & 8 & 9 & 10 & 11 \\
\hline \multicolumn{11}{|c|}{ 178-1096A- } \\
\hline $2 \mathrm{H}$ & 7.89 & 0.113 & 99.00 & 99.00 & 96.11 & 88.03 & 75.57 & 63.11 & 52.34 & 42.77 \\
\hline $2 \mathrm{H}$ & 8.30 & 0.119 & 99.84 & 99.14 & 96.83 & 88.50 & 75.66 & 62.81 & 52.78 & 41.84 \\
\hline $2 \mathrm{H}$ & 8.80 & 0.126 & 99.80 & 99.50 & 97.39 & 88.33 & 76.56 & 64.39 & 54.13 & 44.47 \\
\hline $2 \mathrm{H}$ & 9.29 & 0.133 & 99.80 & 98.69 & 96.89 & 89.55 & 77.99 & 65.83 & 55.38 & 44.02 \\
\hline $2 \mathrm{H}$ & 9.79 & 0.140 & 95.60 & 95.60 & 93.57 & 86.69 & 75.26 & 62.96 & 53.08 & 43.10 \\
\hline $2 \mathrm{H}$ & 10.31 & 0.147 & 99.40 & 99.40 & 96.78 & 88.12 & 75.03 & 62.74 & 52.57 & 42.70 \\
\hline $2 \mathrm{H}$ & 10.80 & 0.154 & 98.80 & 96.90 & 91.02 & 79.44 & 65.57 & 53.99 & 45.21 & 36.63 \\
\hline $2 \mathrm{H}$ & 11.29 & 0.161 & 99.90 & 97.39 & 91.17 & 79.44 & 65.90 & 54.36 & 45.64 & 36.61 \\
\hline $2 \mathrm{H}$ & 11.80 & 0.169 & 81.70 & 80.53 & 75.60 & 66.41 & 56.05 & 47.28 & 39.68 & 32.83 \\
\hline $2 \mathrm{H}$ & 12.29 & 0.176 & 99.40 & 99.10 & 96.59 & 87.75 & 74.40 & 61.65 & 51.21 & 40.86 \\
\hline $2 \mathrm{H}$ & 12.79 & 0.183 & 96.70 & 96.70 & 94.74 & 87.19 & 73.95 & 60.22 & 49.53 & 39.43 \\
\hline $2 \mathrm{H}$ & 13.29 & 0.190 & 97.40 & 96.51 & 92.45 & 82.14 & 65.30 & 51.42 & 41.42 & 33.49 \\
\hline $2 \mathrm{H}$ & 13.53 & 0.193 & 95.50 & 93.94 & 85.94 & 74.43 & 57.94 & 43.41 & 32.19 & 22.44 \\
\hline $2 \mathrm{H}$ & 13.95 & 0.199 & 94.40 & 94.02 & 89.31 & 79.23 & 65.59 & 53.01 & 44.08 & 34.96 \\
\hline $2 \mathrm{H}$ & 14.49 & 0.207 & 97.80 & 95.71 & 89.92 & 78.46 & 64.40 & 52.54 & 43.87 & 35.19 \\
\hline $2 \mathrm{H}$ & 15.00 & 0.214 & 92.10 & 90.89 & 85.94 & 75.29 & 61.65 & 49.51 & 40.63 & 30.64 \\
\hline $2 \mathrm{H}$ & 15.49 & 0.221 & 99.50 & 97.39 & 92.38 & 82.55 & 66.70 & 53.46 & 42.93 & 32.30 \\
\hline $2 \mathrm{H}$ & 15.90 & 0.227 & 89.50 & 89.13 & 86.21 & 73.96 & 57.69 & 45.25 & 35.75 & 26.60 \\
\hline $2 \mathrm{H}$ & 16.46 & 0.235 & 98.70 & 97.81 & 94.23 & 84.78 & 72.06 & 58.74 & 48.41 & 38.66 \\
\hline $3 \mathrm{H}$ & 18.50 & 0.264 & 100.00 & 99.70 & 96.58 & 88.24 & 75.98 & 63.72 & 53.27 & 42.81 \\
\hline $3 \mathrm{H}$ & 19.00 & 0.271 & 99.30 & 97.57 & 92.29 & 81.23 & 67.32 & 55.44 & 45.49 & 35.13 \\
\hline $3 \mathrm{H}$ & 19.50 & 0.279 & 96.90 & 94.65 & 87.62 & 76.78 & 63.59 & 51.87 & 42.39 & 33.02 \\
\hline $3 \mathrm{H}$ & 20.00 & 0.286 & 99.10 & 96.37 & 89.39 & 76.25 & 60.47 & 47.22 & 37.52 & 27.91 \\
\hline $3 \mathrm{H}$ & 20.26 & 0.289 & 94.20 & 92.46 & 88.01 & 75.44 & 56.87 & 41.01 & 29.59 & 20.31 \\
\hline $3 \mathrm{H}$ & 20.40 & 0.291 & 94.70 & 92.59 & 87.51 & 75.34 & 58.76 & 45.72 & 36.42 & 27.13 \\
\hline $3 \mathrm{H}$ & 20.60 & 0.294 & 92.90 & 91.10 & 86.66 & 74.45 & 58.37 & 45.50 & 35.57 & 27.06 \\
\hline $3 \mathrm{H}$ & 21.06 & 0.301 & 100.00 & 98.18 & 91.01 & 77.58 & 67.47 & 57.58 & 49.19 & 39.49 \\
\hline $3 \mathrm{H}$ & 21.50 & 0.307 & 99.84 & 99.74 & 97.00 & 88.37 & 75.69 & 63.72 & 53.67 & 43.32 \\
\hline $3 \mathrm{H}$ & 24.50 & 0.350 & 96.80 & 96.01 & 89.83 & 79.32 & 66.56 & 55.37 & 46.44 & 37.80 \\
\hline $4 \mathrm{H}$ & 28.10 & 0.401 & 99.40 & 97.71 & 91.75 & 81.41 & 69.88 & 59.04 & 49.70 & 41.25 \\
\hline $4 \mathrm{H}$ & 28.70 & 0.410 & 99.60 & 99.40 & 96.38 & 88.42 & 76.84 & 65.16 & 54.68 & 44.81 \\
\hline $4 \mathrm{H}$ & 30.21 & 0.432 & 98.30 & 97.09 & 92.06 & 77.98 & 59.56 & 44.87 & 33.61 & 23.64 \\
\hline $4 \mathrm{H}$ & 31.70 & 0.453 & 99.60 & 99.40 & 95.28 & 86.15 & 74.10 & 62.25 & 52.51 & 42.37 \\
\hline $5 \mathrm{H}$ & 36.78 & 0.525 & 100.00 & 98.57 & 85.25 & 69.16 & 56.05 & 45.80 & 38.01 & 29.92 \\
\hline $5 \mathrm{H}$ & 38.30 & 0.547 & 99.30 & 98.80 & 95.07 & 84.98 & 71.58 & 59.48 & 49.40 & 39.42 \\
\hline $5 \mathrm{H}$ & 39.80 & 0.569 & 99.84 & 99.03 & 96.82 & 87.95 & 74.65 & 62.06 & 52.19 & 42.82 \\
\hline $5 \mathrm{H}$ & 41.30 & 0.590 & 99.50 & 98.70 & 87.23 & 71.63 & 59.16 & 49.20 & 40.54 & 32.19 \\
\hline $5 \mathrm{H}$ & 42.80 & 0.611 & 100.00 & 99.40 & 96.49 & 86.35 & 74.80 & 62.45 & 52.71 & 44.28 \\
\hline $5 \mathrm{H}$ & 44.45 & 0.635 & 98.30 & 96.52 & 84.27 & 67.97 & 55.32 & 45.74 & 38.13 & 30.23 \\
\hline $5 \mathrm{H}$ & 45.30 & 0.647 & 99.60 & 99.10 & 94.80 & 84.89 & 71.97 & 59.66 & 49.75 & 40.24 \\
\hline $6 \mathrm{H}$ & 46.69 & 0.667 & 99.70 & 99.40 & 95.29 & 87.17 & 76.25 & 64.73 & 54.51 & 44.49 \\
\hline $6 \mathrm{H}$ & 47.58 & 0.680 & 99.90 & 99.80 & 95.37 & 85.60 & 73.52 & 61.63 & 51.86 & 42.09 \\
\hline $6 \mathrm{H}$ & 49.09 & 0.701 & 99.80 & 98.11 & 96.31 & 88.93 & 77.97 & 66.40 & 55.63 & 45.26 \\
\hline $6 \mathrm{H}$ & 50.59 & 0.723 & 99.80 & 99.80 & 98.37 & 92.23 & 81.07 & 68.68 & 57.12 & 45.65 \\
\hline $6 \mathrm{H}$ & 52.10 & 0.744 & 100.00 & 100.00 & 97.67 & 89.98 & 78.44 & 66.09 & 55.16 & 44.33 \\
\hline $6 \mathrm{H}$ & 53.60 & 0.766 & 97.70 & 97.70 & 95.87 & 89.78 & 76.58 & 64.19 & 53.72 & 43.67 \\
\hline $7 \mathrm{H}$ & 55.34 & 0.787 & 99.20 & 97.91 & 94.75 & 88.72 & 76.75 & 63.59 & 53.90 & 43.42 \\
\hline $7 \mathrm{H}$ & 55.93 & 0.794 & 96.30 & 94.84 & 89.50 & 80.28 & 67.76 & 55.43 & 45.33 & 35.43 \\
\hline $7 \mathrm{H}$ & 56.84 & 0.804 & 99.65 & 98.85 & 95.15 & 85.66 & 75.36 & 64.37 & 53.87 & 42.88 \\
\hline $7 \mathrm{H}$ & 58.34 & 0.821 & 99.90 & 98.90 & 94.51 & 85.81 & 73.73 & 61.64 & 50.95 & 39.26 \\
\hline $7 \mathrm{H}$ & 59.84 & 0.838 & 99.84 & 99.14 & 95.73 & 87.40 & 76.36 & 64.22 & 54.18 & 44.25 \\
\hline $7 \mathrm{H}$ & 61.31 & 0.854 & 99.40 & 98.80 & 94.49 & 85.67 & 73.45 & 61.42 & 51.50 & 41.88 \\
\hline $7 \mathrm{H}$ & 62.34 & 0.866 & 99.84 & 98.24 & 95.45 & 85.96 & 73.38 & 60.90 & 51.32 & 40.44 \\
\hline $7 \mathrm{H}$ & 63.34 & 0.877 & 98.10 & 97.90 & 91.65 & 80.13 & 66.72 & 55.21 & 45.87 & 37.14 \\
\hline $8 \mathrm{H}$ & 65.10 & 0.897 & 100.00 & 100.00 & 96.06 & 87.07 & 78.08 & 66.77 & 55.96 & 45.35 \\
\hline $8 \mathrm{H}$ & 66.59 & 0.914 & 99.84 & 97.26 & 92.19 & 82.26 & 69.54 & 57.62 & 48.28 & 38.25 \\
\hline $8 \mathrm{H}$ & 68.09 & 0.931 & 99.45 & 98.65 & 95.45 & 86.56 & 74.76 & 63.37 & 53.87 & 44.78 \\
\hline $8 \mathrm{H}$ & 69.59 & 0.947 & 99.90 & 99.40 & 96.19 & 85.27 & 72.75 & 60.92 & 51.50 & 41.78 \\
\hline $8 \mathrm{H}$ & 71.09 & 0.964 & 99.70 & 98.40 & 94.50 & 83.40 & 71.40 & 60.20 & 50.70 & 40.90 \\
\hline $8 \mathrm{H}$ & 72.64 & 0.982 & 99.80 & 99.40 & 97.50 & 88.78 & 76.25 & 63.93 & 52.51 & 41.98 \\
\hline $8 \mathrm{H}$ & 73.78 & 0.994 & 97.20 & 93.15 & 72.90 & 55.51 & 45.34 & 37.83 & 29.34 & 21.14 \\
\hline $9 \mathrm{H}$ & 76.10 & 1.021 & 99.80 & 99.40 & 95.09 & 86.56 & 74.82 & 62.59 & 52.76 & 42.23 \\
\hline $9 \mathrm{H}$ & 77.60 & 1.037 & 99.50 & 99.00 & 94.69 & 84.96 & 72.42 & 59.88 & 50.25 & 39.62 \\
\hline $9 \mathrm{H}$ & 79.88 & 1.063 & 99.84 & 99.84 & 96.91 & 87.61 & 74.88 & 63.56 & 53.56 & 43.45 \\
\hline $9 \mathrm{H}$ & 80.27 & 1.067 & 92.00 & 91.81 & 89.59 & 84.30 & 74.10 & 62.69 & 53.23 & 42.75 \\
\hline $9 \mathrm{H}$ & 80.58 & 1.071 & 97.30 & 96.32 & 94.07 & 86.25 & 68.94 & 52.51 & 40.68 & 28.85 \\
\hline $9 \mathrm{H}$ & 80.87 & 1.074 & 98.50 & 97.41 & 93.63 & 84.29 & 69.87 & 54.87 & 43.44 & 31.91 \\
\hline
\end{tabular}


C.J. Pudsey

DATA Report: Grain-Size DATA

Table T5 (continued).

\begin{tabular}{|c|c|c|c|c|c|c|c|c|c|c|}
\hline \multirow[b]{2}{*}{ Core } & \multirow{2}{*}{$\begin{array}{l}\text { Depth } \\
\text { (mbsf) }\end{array}$} & \multirow{2}{*}{$\begin{array}{l}\text { Age } \\
\text { (Ma) }\end{array}$} & \multicolumn{8}{|c|}{ Grain size $(\phi)$} \\
\hline & & & 4 & 5 & 6 & 7 & 8 & 9 & 10 & 11 \\
\hline $9 \mathrm{H}$ & 81.16 & 1.077 & 98.00 & 97.31 & 94.93 & 88.01 & 76.84 & 64.58 & 54.69 & 44.90 \\
\hline $9 \mathrm{H}$ & 81.45 & 1.081 & 94.30 & 91.94 & 87.79 & 78.08 & 63.09 & 49.22 & 39.32 & 28.67 \\
\hline $9 \mathrm{H}$ & 81.83 & 1.085 & 95.90 & 94.25 & 90.75 & 80.73 & 64.78 & 49.99 & 38.90 & 28.69 \\
\hline $9 \mathrm{H}$ & 82.05 & 1.087 & 96.60 & 93.79 & 86.80 & 76.04 & 61.98 & 50.34 & 41.12 & 32.20 \\
\hline $9 \mathrm{H}$ & 83.13 & 1.100 & 99.90 & 99.30 & 96.29 & 88.68 & 76.95 & 64.73 & 54.61 & 43.69 \\
\hline $10 \mathrm{H}$ & 87.09 & 1.144 & 99.80 & 97.82 & 94.84 & 84.13 & 71.43 & 59.32 & 49.50 & 39.48 \\
\hline $11 \mathrm{H}$ & 94.49 & 1.227 & 99.80 & 99.29 & 95.24 & 87.14 & 74.88 & 63.02 & 53.09 & 42.96 \\
\hline $11 \mathrm{H}$ & 97.39 & 1.260 & 99.80 & 99.60 & 97.57 & 87.86 & 74.39 & 61.64 & 51.82 & 42.41 \\
\hline $11 \mathrm{H}$ & 98.88 & 1.276 & 98.90 & 95.88 & 75.03 & 56.10 & 44.52 & 36.16 & 30.11 & 23.77 \\
\hline $11 \mathrm{H}$ & 100.40 & 1.294 & 99.60 & 99.00 & 97.19 & 89.04 & 77.37 & 65.49 & 55.03 & 44.97 \\
\hline $12 \mathrm{H}$ & 99.64 & 1.285 & 98.30 & 95.99 & 83.05 & 66.90 & 53.66 & 42.33 & 33.60 & 26.38 \\
\hline $12 \mathrm{H}$ & 101.74 & 1.309 & 93.10 & 91.32 & 85.61 & 74.85 & 59.23 & 46.13 & 35.37 & 25.64 \\
\hline $12 \mathrm{H}$ & 104.75 & 1.342 & 99.90 & 99.90 & 96.77 & 87.50 & 74.19 & 62.50 & 52.12 & 42.24 \\
\hline $12 \mathrm{H}$ & 107.75 & 1.376 & 95.80 & 95.61 & 93.07 & 83.91 & 66.76 & 49.70 & 37.52 & 26.61 \\
\hline $13 \mathrm{H}$ & 108.74 & 1.387 & 94.60 & 93.27 & 90.13 & 85.09 & 74.44 & 61.70 & 50.01 & 41.07 \\
\hline $13 \mathrm{H}$ & 109.14 & 1.392 & 97.60 & 95.04 & 90.91 & 81.17 & 70.15 & 58.84 & 48.60 & 37.68 \\
\hline $13 \mathrm{H}$ & 109.59 & 1.397 & 96.60 & 94.26 & 88.89 & 80.60 & 67.42 & 54.15 & 43.03 & 32.88 \\
\hline $13 \mathrm{H}$ & 110.00 & 1.401 & 94.50 & 92.86 & 83.57 & 68.38 & 51.36 & 37.53 & 26.79 & 17.31 \\
\hline $13 \mathrm{H}$ & 110.39 & 1.406 & 99.50 & 99.20 & 95.10 & 85.39 & 71.87 & 59.66 & 49.65 & 41.24 \\
\hline $13 \mathrm{H}$ & 110.80 & 1.410 & 97.40 & 95.83 & 92.50 & 85.15 & 73.49 & 60.07 & 49.48 & 39.00 \\
\hline $13 \mathrm{H}$ & 111.15 & 1.414 & 90.10 & 87.73 & 82.63 & 71.42 & 56.30 & 43.36 & 33.16 & 24.05 \\
\hline $13 \mathrm{H}$ & 111.56 & 1.419 & & & & & & & & \\
\hline $13 \mathrm{H}$ & 112.08 & 1.425 & 99.90 & 98.19 & 92.94 & 80.75 & 67.64 & 56.35 & 47.18 & 38.91 \\
\hline $13 \mathrm{H}$ & 112.49 & 1.429 & 95.00 & 94.32 & 89.33 & 78.58 & 62.55 & 49.16 & 39.29 & 30.30 \\
\hline $13 \mathrm{H}$ & 112.89 & 1.434 & 96.20 & 94.40 & 90.23 & 81.79 & 70.42 & 59.62 & 50.42 & 41.13 \\
\hline $13 \mathrm{H}$ & 113.29 & 1.438 & 100.00 & 99.60 & 97.28 & 88.32 & 76.13 & 63.95 & 53.88 & 43.71 \\
\hline $13 \mathrm{H}$ & 113.69 & 1.443 & 99.90 & 99.30 & 97.19 & 89.77 & 78.44 & 67.10 & 56.77 & 46.54 \\
\hline $13 \mathrm{H}$ & 115.30 & 1.461 & 99.80 & 99.70 & 96.57 & 87.49 & 75.08 & 62.97 & 53.18 & 43.69 \\
\hline \multicolumn{11}{|c|}{ 178-1996C } \\
\hline $1 \mathrm{H}$ & 115.70 & 1.465 & 100.00 & 99.59 & 93.21 & 82.67 & 70.72 & 58.66 & 49.04 & 39.72 \\
\hline $1 \mathrm{H}$ & 120.14 & 1.515 & 99.90 & 99.90 & 99.38 & 92.06 & 78.95 & 64.71 & 53.36 & 43.55 \\
\hline \multicolumn{11}{|c|}{$178-1096 \mathrm{~A}-$} \\
\hline $14 \mathrm{H}$ & 122.80 & 1.545 & 99.20 & 99.10 & 97.80 & 89.78 & 78.06 & 65.73 & 55.11 & 44.69 \\
\hline $14 \mathrm{H}$ & 123.56 & 1.554 & 84.40 & 82.57 & 76.32 & 66.49 & 50.24 & 34.33 & 25.73 & 19.73 \\
\hline $14 \mathrm{H}$ & 125.72 & 1.578 & 100.00 & 99.60 & 96.97 & 89.60 & 77.27 & 65.25 & 55.15 & 45.56 \\
\hline $14 \mathrm{H}$ & 128.70 & 1.612 & 100.00 & 99.39 & 98.38 & 92.32 & 81.72 & 69.70 & 59.49 & 48.89 \\
\hline $15 \mathrm{H}$ & 132.02 & 1.649 & 100.00 & 100.00 & 96.88 & 86.03 & 72.76 & 61.01 & 51.06 & 40.80 \\
\hline $15 \mathrm{H}$ & 134.23 & 1.674 & 99.00 & 95.02 & 76.41 & 56.32 & 44.48 & 37.31 & 30.94 & 24.58 \\
\hline $15 \mathrm{H}$ & 134.75 & 1.680 & 99.00 & 98.70 & 95.58 & 86.22 & 75.16 & 63.99 & 53.83 & 44.37 \\
\hline $15 \mathrm{H}$ & 137.75 & 1.713 & 100.00 & 99.80 & 95.55 & 87.15 & 74.90 & 62.85 & 53.34 & 43.12 \\
\hline \multicolumn{11}{|c|}{ 178-1096B- } \\
\hline $16 \mathrm{H}$ & 136.50 & 1.699 & 100.00 & 99.80 & 96.74 & 86.88 & 74.87 & 63.28 & 53.10 & 43.23 \\
\hline $16 \mathrm{H}$ & 137.20 & 1.707 & 98.80 & 98.30 & 93.68 & 83.95 & 70.82 & 58.88 & 49.35 & 39.62 \\
\hline $16 \mathrm{H}$ & 137.30 & 1.708 & 95.00 & 94.90 & 92.00 & 83.58 & 68.59 & 55.05 & 45.18 & 35.89 \\
\hline $16 \mathrm{H}$ & 137.40 & 1.709 & 94.10 & 92.48 & 88.87 & 79.54 & 64.70 & 51.76 & 42.53 & 33.40 \\
\hline $16 \mathrm{H}$ & 137.50 & 1.710 & 89.60 & 88.06 & 83.80 & 75.36 & 63.03 & 51.87 & 42.71 & 33.37 \\
\hline $16 \mathrm{H}$ & 137.60 & 1.712 & 96.70 & 96.02 & 91.72 & 83.22 & 70.23 & 58.31 & 49.33 & 41.02 \\
\hline $16 \mathrm{H}$ & 137.70 & 1.713 & 99.40 & 99.20 & 97.19 & 91.06 & 79.60 & 67.14 & 56.48 & 46.03 \\
\hline $16 \mathrm{H}$ & 138.00 & 1.716 & 99.60 & 99.20 & 97.89 & 91.56 & 80.50 & 67.94 & 57.39 & 46.94 \\
\hline $16 \mathrm{H}$ & 139.50 & 1.733 & 99.90 & 99.80 & 96.29 & 88.68 & 77.35 & 65.93 & 55.91 & 46.39 \\
\hline $16 \mathrm{H}$ & 141.00 & 1.750 & 99.60 & 98.59 & 94.96 & 87.70 & 76.51 & 63.41 & 53.23 & 42.44 \\
\hline $16 \mathrm{H}$ & 142.50 & 1.767 & 99.60 & 98.99 & 96.57 & 88.89 & 77.17 & 65.15 & 54.95 & 44.95 \\
\hline $16 \mathrm{H}$ & 143.40 & 1.777 & 99.90 & 99.80 & 97.37 & 89.80 & 79.09 & 67.37 & 57.37 & 46.77 \\
\hline $17 \mathrm{H}$ & 144.60 & 1.790 & 99.70 & 99.30 & 96.18 & 88.72 & 77.14 & 64.96 & 54.28 & 43.10 \\
\hline $17 \mathrm{H}$ & 146.10 & 1.807 & 99.90 & 99.90 & 96.96 & 89.04 & 77.87 & 66.09 & 55.64 & 43.96 \\
\hline $17 \mathrm{H}$ & 147.60 & 1.824 & 99.90 & 99.10 & 95.28 & 87.05 & 75.70 & 63.86 & 54.12 & 42.57 \\
\hline $17 \mathrm{H}$ & 149.10 & 1.841 & 99.86 & 98.85 & 95.43 & 86.77 & 75.10 & 63.12 & 52.75 & 42.28 \\
\hline $18 \mathrm{H}$ & 150.26 & 1.854 & 91.60 & 90.32 & 87.75 & 81.15 & 71.34 & 60.52 & 51.16 & 41.63 \\
\hline $18 \mathrm{H}$ & 150.45 & 1.856 & 98.90 & 98.80 & 95.79 & 88.16 & 78.12 & 65.87 & 55.52 & 45.99 \\
\hline $19 \mathrm{H}$ & 151.79 & 1.871 & 99.90 & 99.00 & 97.39 & 90.58 & 80.06 & 68.24 & 58.12 & 47.90 \\
\hline $19 \mathrm{H}$ & 153.29 & 1.888 & 99.60 & 99.60 & 96.99 & 89.87 & 78.64 & 67.30 & 56.87 & 46.74 \\
\hline $19 \mathrm{H}$ & 154.48 & 1.901 & 99.80 & 97.89 & 93.78 & 85.24 & 73.59 & 61.95 & 52.21 & 41.97 \\
\hline $19 \mathrm{H}$ & 156.29 & 1.922 & 99.40 & 99.40 & 97.69 & 90.55 & 79.78 & 67.00 & 56.64 & 45.47 \\
\hline $19 \mathrm{H}$ & 156.99 & 1.929 & 99.86 & 98.15 & 94.52 & 84.76 & 72.48 & 60.60 & 50.63 & 41.17 \\
\hline $20 \mathrm{H}$ & 158.09 & 1.942 & 99.86 & 99.86 & 96.92 & 89.62 & 77.66 & 65.19 & 54.64 & 44.30 \\
\hline $2 \mathrm{OH}$ & 159.59 & 1.959 & 99.60 & 99.30 & 95.57 & 86.60 & 74.50 & 62.40 & 52.22 & 42.74 \\
\hline $20 \mathrm{H}$ & 161.09 & 1.975 & 99.86 & 99.25 & 96.02 & 87.62 & 77.20 & 64.65 & 53.62 & 41.48 \\
\hline $20 \mathrm{H}$ & 164.09 & 2.009 & 97.40 & 95.46 & 93.12 & 87.87 & 79.61 & 68.34 & 58.32 & 48.12 \\
\hline $22 x$ & 167.29 & 2.045 & 95.60 & 95.12 & 92.81 & 84.90 & 69.58 & 53.20 & 40.76 & 29.10 \\
\hline
\end{tabular}


C.J. Pudsey

DATA Report: Grain-Size DATA

Table T5 (continued).

\begin{tabular}{|c|c|c|c|c|c|c|c|c|c|c|}
\hline \multirow[b]{2}{*}{ Core } & \multirow{2}{*}{$\begin{array}{l}\text { Depth } \\
\text { (mbsf) }\end{array}$} & \multirow{2}{*}{$\begin{array}{l}\text { Age } \\
(\mathrm{Ma})\end{array}$} & \multicolumn{8}{|c|}{ Grain size $(\phi)$} \\
\hline & & & 4 & 5 & 6 & 7 & 8 & 9 & 10 & 11 \\
\hline \multicolumn{11}{|c|}{ 178-1096C- } \\
\hline $2 \mathrm{H}$ & 168.24 & 2.056 & 98.60 & 98.10 & 94.70 & 85.01 & 73.03 & 61.04 & 50.45 & 40.86 \\
\hline $2 \mathrm{H}$ & 169.90 & 2.074 & 99.90 & 99.90 & 98.66 & 90.82 & 77.61 & 64.81 & 53.97 & 42.83 \\
\hline $2 \mathrm{H}$ & 171.46 & 2.092 & 99.90 & 99.90 & 98.67 & 92.40 & 78.03 & 65.30 & 54.52 & 43.53 \\
\hline $2 \mathrm{H}$ & 173.03 & 2.110 & 99.90 & 99.90 & 98.56 & 91.88 & 80.37 & 67.73 & 56.43 & 46.15 \\
\hline $2 \mathrm{H}$ & 173.81 & 2.118 & 89.90 & 89.17 & 84.77 & 75.71 & 64.45 & 52.91 & 43.12 & 33.32 \\
\hline $2 \mathrm{H}$ & 174.61 & 2.127 & 98.50 & 95.33 & 87.30 & 76.10 & 62.23 & 50.64 & 41.42 & 32.30 \\
\hline \multicolumn{11}{|c|}{ 178-1096B- } \\
\hline $23 x$ & 174.70 & 2.128 & 95.60 & 95.50 & 91.63 & 82.91 & 71.87 & 60.05 & 50.27 & 40.87 \\
\hline \multicolumn{11}{|c|}{ 178-1096C- } \\
\hline $2 \mathrm{H}$ & 176.16 & 2.145 & 100.00 & 100.00 & 97.57 & 89.07 & 77.23 & 64.17 & 53.54 & 43.32 \\
\hline \multicolumn{11}{|c|}{ 178-1096B- } \\
\hline $23 X$ & 176.24 & 2.146 & 99.90 & 99.30 & 96.57 & 87.10 & 74.70 & 62.30 & 52.42 & 42.14 \\
\hline \multicolumn{11}{|c|}{ 178-1096C- } \\
\hline $2 \mathrm{H}$ & 176.56 & 2.149 & 99.80 & 99.30 & 95.78 & 88.05 & 75.30 & 62.55 & 52.01 & 41.37 \\
\hline \multicolumn{11}{|c|}{ 178-1096B- } \\
\hline $23 x$ & 177.64 & 2.161 & 99.90 & 98.70 & 95.40 & 86.61 & 75.22 & 62.94 & 52.45 & 41.56 \\
\hline $23 x$ & 179.15 & 2.178 & 99.90 & 99.90 & 97.57 & 89.47 & 78.04 & 66.70 & 56.17 & 45.85 \\
\hline $24 X$ & 184.09 & 2.234 & 100.00 & 99.49 & 97.67 & 90.19 & 78.16 & 65.12 & 54.80 & 45.80 \\
\hline $24 X$ & 185.50 & 2.250 & 99.90 & 99.70 & 96.44 & 87.81 & 76.22 & 64.33 & 54.17 & 45.02 \\
\hline $24 X$ & 187.00 & 2.267 & 100.00 & 99.19 & 96.37 & 87.20 & 74.19 & 60.89 & 50.40 & 40.12 \\
\hline $24 X$ & 188.50 & 2.283 & 99.60 & 98.59 & 93.85 & 84.08 & 71.37 & 58.77 & 48.99 & 38.91 \\
\hline $24 X$ & 190.00 & 2.300 & 97.50 & 95.34 & 88.66 & 77.08 & 63.72 & 51.84 & 43.30 & 34.17 \\
\hline $24 X$ & 191.50 & 2.317 & 99.70 & 98.90 & 94.88 & 85.53 & 72.66 & 60.30 & 49.65 & 38.69 \\
\hline $24 X$ & 192.42 & 2.327 & 99.90 & 98.88 & 93.50 & 82.44 & 69.24 & 57.36 & 47.31 & 37.97 \\
\hline $178-1$ & - & & & & & & & & & \\
\hline $3 x$ & 195.80 & 2.365 & 99.10 & 98.80 & 95.28 & 84.84 & 71.39 & 59.04 & 48.60 & 38.46 \\
\hline $3 x$ & 198.80 & 2.399 & 95.60 & 95.03 & 90.14 & 81.71 & 70.41 & 58.62 & 48.37 & 38.41 \\
\hline $3 x$ & 200.29 & 2.416 & 86.40 & 86.05 & 83.45 & 78.93 & 69.12 & 55.40 & 43.42 & 32.48 \\
\hline $3 x$ & 200.69 & 2.420 & 99.70 & 99.70 & 98.56 & 92.98 & 81.50 & 67.95 & 56.16 & 44.99 \\
\hline $3 X$ & 201.85 & 2.433 & 99.90 & 99.90 & 98.45 & 91.61 & 79.07 & 65.29 & 53.47 & 43.32 \\
\hline $178-1$ & $B-$ & & & & & & & & & \\
\hline $26 X$ & 203.19 & 2.449 & 100.00 & 99.60 & 96.97 & 87.97 & 74.92 & 60.97 & 50.35 & 40.65 \\
\hline $26 X$ & 204.69 & 2.465 & 99.90 & 99.80 & 96.86 & 87.45 & 73.69 & 61.34 & 50.51 & 42.00 \\
\hline $26 X$ & 206.19 & 2.482 & 99.90 & 99.39 & 95.15 & 86.16 & 74.24 & 62.12 & 51.31 & 40.81 \\
\hline $26 X$ & 207.69 & 2.499 & 99.90 & 99.60 & 96.37 & 87.40 & 75.50 & 62.70 & 51.81 & 42.04 \\
\hline $26 X$ & 209.19 & 2.516 & 99.90 & 98.69 & 95.88 & 86.83 & 75.78 & 63.82 & 53.47 & 43.42 \\
\hline $26 X$ & 210.69 & 2.533 & 99.74 & 99.34 & 94.18 & 82.34 & 68.99 & 57.15 & 47.04 & 36.42 \\
\hline $26 X$ & 211.94 & 2.547 & 98.60 & 97.81 & 94.64 & 84.53 & 71.75 & 60.05 & 49.85 & 40.23 \\
\hline $27 x$ & 213.70 & 2.567 & 99.90 & 99.70 & 96.25 & 86.80 & 74.93 & 62.95 & 52.59 & 42.03 \\
\hline $27 x$ & 215.19 & 2.583 & 99.90 & 99.20 & 95.58 & 88.05 & 76.31 & 63.76 & 53.41 & 43.27 \\
\hline $27 x$ & 216.69 & 2.585 & 99.90 & 99.50 & 93.68 & 79.74 & 66.40 & 55.27 & 45.94 & 36.81 \\
\hline $27 x$ & 217.59 & 2.590 & 98.90 & 98.30 & 94.48 & 87.94 & 77.29 & 65.83 & 55.48 & 45.73 \\
\hline $28 X$ & 223.29 & 2.618 & 99.00 & 96.90 & 89.10 & 78.70 & 67.50 & 56.20 & 46.90 & 37.90 \\
\hline $28 X$ & 224.79 & 2.625 & 99.84 & 99.84 & 96.81 & 88.42 & 76.50 & 64.07 & 53.36 & 42.44 \\
\hline $28 X$ & 226.05 & 2.632 & 99.40 & 97.49 & 92.15 & 81.98 & 69.99 & 58.01 & 48.04 & 37.56 \\
\hline $29 X$ & 232.29 & 2.663 & 99.84 & 99.54 & 97.01 & 87.52 & 75.31 & 62.89 & 52.60 & 42.60 \\
\hline $29 X$ & 233.79 & 2.670 & 99.70 & 98.99 & 93.11 & 82.07 & 69.30 & 57.55 & 47.92 & 39.72 \\
\hline $29 X$ & 235.30 & 2.678 & 99.60 & 98.99 & 93.63 & 83.42 & 70.68 & 58.45 & 47.93 & 38.32 \\
\hline $29 x$ & 236.80 & 2.685 & 99.80 & 99.80 & 97.18 & 89.53 & 77.34 & 65.16 & 54.78 & 44.51 \\
\hline $29 X$ & 238.29 & 2.693 & 99.90 & 99.40 & 96.49 & 88.68 & 77.46 & 65.23 & 55.31 & 45.09 \\
\hline $30 x$ & 242.29 & 2.713 & 99.90 & 99.30 & 95.21 & 86.33 & 74.55 & 63.17 & 52.69 & 42.32 \\
\hline $30 x$ & 243.79 & 2.720 & 100.00 & 99.70 & 96.67 & 87.70 & 75.60 & 63.71 & 53.02 & 41.63 \\
\hline $30 x$ & 244.50 & 2.724 & 100.00 & 99.49 & 98.27 & 92.04 & 79.69 & 67.24 & 56.02 & 43.88 \\
\hline $30 x$ & 245.10 & 2.727 & 100.00 & 99.90 & 96.95 & 88.82 & 76.73 & 64.23 & 53.25 & 43.19 \\
\hline $30 x$ & 245.69 & 2.729 & 99.90 & 99.90 & 98.46 & 92.30 & 80.70 & 67.66 & 56.47 & 45.18 \\
\hline $30 x$ & 245.90 & 2.731 & 99.30 & 99.30 & 96.83 & 89.19 & 76.51 & 63.21 & 51.66 & 40.73 \\
\hline $30 x$ & 246.10 & 2.731 & 88.70 & 86.29 & 81.55 & 72.98 & 61.90 & 50.02 & 39.93 & 29.21 \\
\hline $30 x$ & 246.29 & 2.732 & 98.50 & 96.82 & 91.70 & 83.41 & 70.40 & 57.58 & 47.43 & 36.28 \\
\hline $30 x$ & 246.49 & 2.733 & 98.20 & 97.30 & 93.72 & 85.56 & 73.82 & 60.99 & 50.05 & 37.51 \\
\hline $30 x$ & 246.70 & 2.734 & 99.30 & 98.06 & 96.93 & 89.10 & 75.51 & 61.70 & 51.61 & 41.10 \\
\hline $30 x$ & 246.87 & 2.735 & 100.00 & 98.69 & 96.18 & 88.15 & 76.71 & 64.76 & 54.72 & 43.57 \\
\hline $31 x$ & 248.70 & 2.744 & 99.90 & 99.70 & 96.48 & 89.14 & 77.67 & 66.90 & 56.04 & 45.57 \\
\hline $32 x$ & 251.39 & 2.758 & 99.90 & 99.30 & 93.98 & 84.64 & 73.19 & 62.25 & 52.51 & 42.77 \\
\hline $32 x$ & 254.39 & 2.773 & 99.90 & 99.90 & 96.87 & 88.38 & 76.06 & 64.95 & 54.75 & 45.05 \\
\hline $32 x$ & 256.84 & 2.785 & 99.90 & 99.80 & 97.69 & 90.24 & 78.47 & 66.90 & 56.44 & 45.98 \\
\hline
\end{tabular}


C.J. Pudsey

DATA Report: Grain-Size DATA

Table T6. Median diameter, mode, sorting, and skewness, Site 1096. (Continued on next page.)

\begin{tabular}{|c|c|c|c|c|c|c|}
\hline Core & $\begin{array}{l}\text { Depth } \\
\text { (mbsf) }\end{array}$ & $\begin{array}{l}\text { Age } \\
(\mathrm{Ma})\end{array}$ & $\phi 50$ & Sorting & Skewness & $\begin{array}{c}\text { Mode } \\
(\phi)\end{array}$ \\
\hline \multicolumn{7}{|c|}{ 178-1096A- } \\
\hline $2 \mathrm{H}$ & 7.89 & 0.113 & 10.25 & 3.075 & 0.073 & 7.8 \\
\hline $2 \mathrm{H}$ & 8.30 & 0.119 & 10.25 & 2.95 & 0.034 & 7.9 \\
\hline $2 \mathrm{H}$ & 8.80 & 0.126 & 10.45 & 3.225 & 0.054 & 7.9 \\
\hline $2 \mathrm{H}$ & 9.29 & 0.133 & 10.45 & 3.025 & 0.025 & \\
\hline $2 \mathrm{H}$ & 9.79 & 0.140 & 10.3 & 3.2 & 0.062 & 7.9 \\
\hline $2 \mathrm{H}$ & 10.31 & 0.147 & 10.3 & 3.125 & 0.056 & 7.9 \\
\hline $2 \mathrm{H}$ & 10.80 & 0.154 & 9.45 & 3.425 & 0.182 & 7.9 \\
\hline $2 \mathrm{H}$ & 11.29 & 0.161 & 9.5 & 3.4 & 0.147 & 7.8 \\
\hline $2 \mathrm{H}$ & 11.80 & 0.169 & 8.65 & 5.25 & -0.076 & 7.8 \\
\hline $2 \mathrm{H}$ & 12.29 & 0.176 & 10.1 & 3.05 & 0.082 & 7.9 \\
\hline $2 \mathrm{H}$ & 12.79 & 0.183 & 9.9 & 3.025 & 0.140 & 7.9 \\
\hline $2 \mathrm{H}$ & 13.29 & 0.190 & 9.1 & 3.175 & 0.291 & 7.9 \\
\hline $2 \mathrm{H}$ & 13.53 & 0.193 & 8.55 & 2.7 & 0.130 & 7.7 \\
\hline $2 \mathrm{H}$ & 13.95 & 0.199 & 9.35 & 3.275 & 0.145 & 7.9 \\
\hline $2 \mathrm{H}$ & 14.49 & 0.207 & 9.25 & 3.375 & 0.200 & 7.9 \\
\hline $2 \mathrm{H}$ & 15.00 & 0.214 & 8.95 & 3.2 & 0.141 & 7.9 \\
\hline $2 \mathrm{H}$ & 15.49 & 0.221 & 9.3 & 2.85 & 0.158 & 7.8 \\
\hline $2 \mathrm{H}$ & 15.90 & 0.227 & 8.55 & 3 & 0.233 & 7.5 \\
\hline $2 \mathrm{H}$ & 16.46 & 0.235 & 9.05 & 3.175 & 0.370 & 8 \\
\hline $3 \mathrm{H}$ & 18.50 & 0.264 & 10.35 & 3.125 & 0.056 & 7.9 \\
\hline $3 \mathrm{H}$ & 19.00 & 0.271 & 9.5 & 3.1 & 0.113 & 7.9 \\
\hline $3 \mathrm{H}$ & 19.50 & 0.279 & 9.2 & 3.25 & 0.123 & 7.8 \\
\hline $3 \mathrm{H}$ & 20.00 & 0.286 & 8.75 & 2.925 & 0.214 & 7.5 \\
\hline $3 \mathrm{H}$ & 20.26 & 0.289 & 8.4 & 2.525 & 0.208 & 7.8 \\
\hline $3 \mathrm{H}$ & 20.40 & 0.291 & 8.65 & 2.975 & 0.227 & 7.6 \\
\hline $3 \mathrm{H}$ & 20.60 & 0.294 & 8.6 & 3.05 & 0.230 & 7.7 \\
\hline $3 \mathrm{H}$ & 21.06 & 0.301 & 9.9 & 3.55 & 0.042 & 6.6 \\
\hline $3 \mathrm{H}$ & 21.50 & 0.307 & 10.4 & 3.1 & 0.032 & 7.8 \\
\hline $3 \mathrm{H}$ & 24.50 & 0.350 & 9.6 & 3.475 & 0.122 & \\
\hline $4 \mathrm{H}$ & 28.10 & 0.401 & 9.95 & 3.625 & 0.117 & \\
\hline $4 \mathrm{H}$ & 28.70 & 0.410 & 10.5 & 3.25 & 0.046 & \\
\hline $4 \mathrm{H}$ & 30.21 & 0.432 & 8.6 & 2.625 & 0.219 & 7.9 \\
\hline $4 \mathrm{H}$ & 31.70 & 0.453 & 10.25 & 3.225 & 0.054 & 7.9 \\
\hline $5 \mathrm{H}$ & 36.78 & 0.525 & 8.55 & 3.35 & 0.269 & 5.9 \\
\hline $5 \mathrm{H}$ & 38.30 & 0.547 & 9.9 & 3.125 & 0.104 & 7.5 \\
\hline $5 \mathrm{H}$ & 39.80 & 0.569 & 10.25 & 3.15 & 0.079 & 7.7 \\
\hline $5 \mathrm{H}$ & 41.30 & 0.590 & 8.9 & 3.375 & 0.185 & 6.1 \\
\hline $5 \mathrm{H}$ & 42.80 & 0.611 & 10.3 & 3.475 & 0.122 & 8.1 \\
\hline $5 \mathrm{H}$ & 44.45 & 0.635 & 8.5 & 3.425 & 0.270 & 6.3 \\
\hline $5 \mathrm{H}$ & 45.30 & 0.647 & 9.95 & 3.25 & 0.123 & 7.9 \\
\hline $6 \mathrm{H}$ & 46.69 & 0.667 & 10.45 & 3.25 & 0.031 & \\
\hline $6 \mathrm{H}$ & 47.58 & 0.680 & 10.2 & 3.225 & 0.054 & 7.9 \\
\hline $6 \mathrm{H}$ & 49.09 & 0.701 & 10.55 & 3.15 & 0.032 & \\
\hline $6 \mathrm{H}$ & 50.59 & 0.723 & 10.6 & 2.85 & 0.018 & \\
\hline $6 \mathrm{H}$ & 52.10 & 0.744 & 10.5 & 2.975 & 0.008 & \\
\hline $6 \mathrm{H}$ & 53.60 & 0.766 & 10.35 & 3.075 & 0.089 & 7.9 \\
\hline $7 \mathrm{H}$ & 55.34 & 0.791 & 10.4 & 3.2 & 0.094 & 8 \\
\hline $7 \mathrm{H}$ & 55.93 & 0.794 & 9.55 & 3.15 & 0.079 & 8 \\
\hline $7 \mathrm{H}$ & 56.84 & 0.804 & 10.35 & 3.125 & -0.008 & \\
\hline $7 \mathrm{H}$ & 58.34 & 0.821 & 10.1 & 2.9 & 0.000 & \\
\hline $7 \mathrm{H}$ & 59.84 & 0.838 & 10.4 & 3.225 & 0.054 & 8.1 \\
\hline $7 \mathrm{H}$ & 61.31 & 0.854 & 10.15 & 3.15 & 0.048 & 8 \\
\hline $7 \mathrm{H}$ & 62.34 & 0.866 & 10.1 & 3.075 & 0.057 & 7.9 \\
\hline $7 \mathrm{H}$ & 63.34 & 0.877 & 9.55 & 3.35 & 0.149 & \\
\hline $8 \mathrm{H}$ & 65.10 & 0.897 & 10.6 & 3.2 & -0.016 & \\
\hline $8 \mathrm{H}$ & 66.59 & 0.914 & 9.8 & 3.2 & 0.062 & 8 \\
\hline $8 \mathrm{H}$ & 68.09 & 0.931 & 10.4 & 3.425 & 0.080 & 8.1 \\
\hline $8 \mathrm{H}$ & 69.59 & 0.947 & 10.15 & 3.275 & 0.069 & \\
\hline $8 \mathrm{H}$ & 71.09 & 0.964 & 10.05 & 3.275 & 0.053 & \\
\hline $8 \mathrm{H}$ & 72.64 & 0.982 & 10.25 & 2.975 & 0.042 & 8 \\
\hline $8 \mathrm{H}$ & 73.78 & 0.994 & 7.5 & 3.025 & 0.355 & 5.6 \\
\hline $9 \mathrm{H}$ & 76.10 & 1.021 & 10.25 & 3.075 & 0.024 & 8.1 \\
\hline $9 \mathrm{H}$ & 77.60 & 1.037 & 10 & 3.075 & 0.057 & 8 \\
\hline $9 \mathrm{H}$ & 79.88 & 1.063 & 10.35 & 3.175 & 0.039 & 7.4 \\
\hline $9 \mathrm{H}$ & 80.27 & 1.067 & 10.3 & 3.275 & 0.008 & 8 \\
\hline $9 \mathrm{H}$ & 80.58 & 1.071 & 9.2 & 2.425 & 0.175 & 7.9 \\
\hline $9 \mathrm{H}$ & 80.87 & 1.074 & 9.4 & 2.7 & 0.111 & 8.2 \\
\hline $9 \mathrm{H}$ & 81.16 & 1.077 & 10.5 & 3.25 & 0.062 & 8.2 \\
\hline $9 \mathrm{H}$ & 81.45 & 1.081 & 8.9 & 2.9 & 0.155 & 8 \\
\hline
\end{tabular}

\begin{tabular}{|c|c|c|c|c|c|c|}
\hline Core & $\begin{array}{l}\text { Depth } \\
\text { (mbsf) }\end{array}$ & $\begin{array}{l}\text { Age } \\
(\mathrm{Ma})\end{array}$ & $\phi 50$ & Sorting & Skewness & $\begin{array}{c}\text { Mode } \\
(\phi)\end{array}$ \\
\hline $9 \mathrm{H}$ & 81.83 & 1.085 & 9 & 2.775 & 0.189 & 7.9 \\
\hline $9 \mathrm{H}$ & 82.05 & 1.087 & 9 & 3.275 & 0.176 & 7.6 \\
\hline $9 \mathrm{H}$ & 83.13 & 1.100 & 10.4 & 3.05 & 0.016 & 8.1 \\
\hline $10 \mathrm{H}$ & 87.09 & 1.144 & 10.95 & 3.15 & -0.254 & 8.1 \\
\hline $11 \mathrm{H}$ & 94.49 & 1.227 & 10.3 & 3.175 & 0.055 & 8 \\
\hline $11 \mathrm{H}$ & 97.39 & 1.260 & 10.2 & 3.15 & 0.095 & 7.9 \\
\hline $11 \mathrm{H}$ & 98.88 & 1.276 & 7.55 & 3.325 & 0.429 & 5.6 \\
\hline $11 \mathrm{H}$ & 100.40 & 1.294 & 10.5 & 3.15 & 0.048 & 8 \\
\hline \multicolumn{7}{|c|}{ 178-1096- } \\
\hline $12 \mathrm{H}$ & 99.64 & 1.285 & 8.25 & 3.25 & 0.292 & 5.9 \\
\hline $12 \mathrm{H}$ & 101.74 & 1.309 & 8.7 & 2.9 & 0.138 & 7.8 \\
\hline $12 \mathrm{H}$ & 104.75 & 1.342 & 10.2 & 3.15 & 0.079 & 7.6 \\
\hline $12 \mathrm{H}$ & 107.75 & 1.376 & 8.95 & 2.5 & 0.220 & 8 \\
\hline $13 \mathrm{H}$ & 108.74 & 1.387 & 10 & 3.3 & 0.136 & \\
\hline $13 \mathrm{H}$ & 109.14 & 1.392 & 9.85 & 3.125 & 0.008 & \\
\hline $13 \mathrm{H}$ & 109.59 & 1.397 & 9.35 & 3 & 0.100 & 7.9 \\
\hline $13 \mathrm{H}$ & 110.00 & 1.401 & 8.1 & 2.55 & 0.176 & 7.3 \\
\hline $13 \mathrm{H}$ & 110.39 & 1.406 & 9.95 & 3.45 & 0.174 & 7.9 \\
\hline $13 \mathrm{H}$ & 110.80 & 1.410 & 9.95 & 3.025 & 0.058 & 7.9 \\
\hline $13 \mathrm{H}$ & 111.15 & 1.414 & 8.45 & 3.075 & 0.138 & 7.6 \\
\hline $13 \mathrm{H}$ & 112.08 & 1.425 & 9.6 & 3.5 & 0.186 & \\
\hline $13 \mathrm{H}$ & 112.49 & 1.429 & 8.9 & 3.05 & 0.230 & 7.9 \\
\hline $13 \mathrm{H}$ & 112.89 & 1.434 & 10 & 3.475 & 0.065 & 8 \\
\hline $13 \mathrm{H}$ & 113.29 & 1.438 & 10.35 & 3.125 & 0.072 & 7.9 \\
\hline $13 \mathrm{H}$ & 113.69 & 1.443 & 10.6 & 3.175 & 0.039 & 8 \\
\hline $13 \mathrm{H}$ & 115.30 & 1.461 & 10.3 & 3.225 & 0.085 & 7.9 \\
\hline \multicolumn{7}{|c|}{ 178-1096C- } \\
\hline $1 \mathrm{H}$ & 115.70 & 1.465 & 9.9 & 3.3 & 0.091 & \\
\hline $1 \mathrm{H}$ & 120.14 & 1.515 & 10.3 & 3.075 & 0.138 & 8.1 \\
\hline $14 \mathrm{H}$ & 122.80 & 1.545 & 10.5 & 3.05 & 0.033 & 8.2 \\
\hline $14 \mathrm{H}$ & 123.56 & 1.554 & 8 & 3.55 & 0.014 & 7.9 \\
\hline $14 \mathrm{H}$ & 125.72 & 1.578 & 10.55 & 3.3 & 0.076 & 7.9 \\
\hline $14 \mathrm{H}$ & 128.70 & 1.612 & 10.9 & 3.15 & 0.016 & 8.2 \\
\hline \multicolumn{7}{|c|}{ 178-1096A- } \\
\hline $15 \mathrm{H}$ & 132.02 & 1.649 & 10.1 & 3.125 & 0.072 & \\
\hline $15 \mathrm{H}$ & 134.23 & 1.674 & 7.5 & 3.35 & 0.463 & 5.9 \\
\hline $15 \mathrm{H}$ & 134.75 & 1.680 & 10.4 & 3.4 & 0.059 & 8 \\
\hline $15 \mathrm{H}$ & 137.75 & 1.713 & 10.3 & 3.225 & 0.054 & 8.1 \\
\hline \multicolumn{7}{|c|}{ 178-1096B- } \\
\hline $16 \mathrm{H}$ & 136.50 & 1.699 & 10.3 & 3.225 & 0.054 & \\
\hline $16 \mathrm{H}$ & 137.20 & 1.707 & 9.9 & 3.225 & 0.101 & 7.9 \\
\hline $16 \mathrm{H}$ & 137.30 & 1.708 & 9.5 & 3.075 & 0.187 & 7.9 \\
\hline $16 \mathrm{H}$ & 137.40 & 1.709 & 9.15 & 3.15 & 0.190 & 7.8 \\
\hline $16 \mathrm{H}$ & 137.50 & 1.710 & 9.2 & 3.425 & 0.066 & 7.4 \\
\hline $16 \mathrm{H}$ & 137.60 & 1.712 & 9.9 & 3.525 & 0.163 & 8 \\
\hline $16 \mathrm{H}$ & 137.70 & 1.713 & 10.6 & 3.05 & 0.049 & 8.3 \\
\hline $16 \mathrm{H}$ & 138.00 & 1.716 & 10.7 & 3.15 & 0.048 & 8.4 \\
\hline $16 \mathrm{H}$ & 139.50 & 1.733 & 10.6 & 3.325 & 0.053 & 8.1 \\
\hline $16 \mathrm{H}$ & 141.00 & 1.750 & 10.3 & 3.05 & 0.049 & 8.1 \\
\hline $16 \mathrm{H}$ & 142.50 & 1.767 & 10.5 & 3.175 & 0.039 & 8.1 \\
\hline $16 \mathrm{H}$ & 143.40 & 1.777 & 10.7 & 3.2 & 0.031 & 8.1 \\
\hline $17 \mathrm{H}$ & 144.60 & 1.790 & 10.4 & 3.025 & 0.008 & \\
\hline $17 \mathrm{H}$ & 146.10 & 1.807 & 10.5 & 3.025 & 0.008 & \\
\hline $17 \mathrm{H}$ & 147.60 & 1.824 & 10.35 & 3.075 & 0.008 & \\
\hline $17 \mathrm{H}$ & 149.10 & 1.841 & 10.25 & 3.15 & 0.048 & \\
\hline $18 \mathrm{H}$ & 150.26 & 1.854 & 10.1 & 3.5 & 0.029 & \\
\hline $18 \mathrm{H}$ & 150.45 & 1.856 & 10.55 & 3.25 & 0.062 & 8.1 \\
\hline $19 \mathrm{H}$ & 151.79 & 1.871 & 10.75 & 3.25 & 0.062 & \\
\hline $19 \mathrm{H}$ & 153.29 & 1.888 & 10.7 & 3.275 & 0.038 & \\
\hline $19 \mathrm{H}$ & 154.48 & 1.901 & 10.2 & 3.225 & 0.039 & \\
\hline $19 \mathrm{H}$ & 156.29 & 1.922 & 10.65 & 3.075 & 0.024 & 8.1 \\
\hline $19 \mathrm{H}$ & 156.99 & 1.929 & 10.05 & 3.275 & 0.084 & 7.9 \\
\hline $20 \mathrm{H}$ & 158.09 & 1.942 & 10.45 & 3.125 & 0.056 & 7.9 \\
\hline $20 \mathrm{H}$ & 159.59 & 1.959 & 10.25 & 3.275 & 0.084 & 7.9 \\
\hline $20 \mathrm{H}$ & 161.09 & 1.975 & 10.3 & 2.875 & -0.009 & \\
\hline $20 \mathrm{H}$ & 164.09 & 2.009 & 10.8 & 3.35 & 0.015 & \\
\hline $22 x$ & 167.29 & 2.045 & 9.25 & 2.6 & 0.154 & 8.1 \\
\hline
\end{tabular}


C.J. Pudsey

DATA Report: Grain-Size DATA

Table T6 (continued).

\begin{tabular}{|c|c|c|c|c|c|c|}
\hline Core & $\begin{array}{l}\text { Depth } \\
\text { (mbsf) }\end{array}$ & $\begin{array}{l}\text { Age } \\
(\mathrm{Ma})\end{array}$ & $\phi 50$ & Sorting & Skewness & $\begin{array}{c}\text { Mode } \\
(\phi)\end{array}$ \\
\hline \multicolumn{7}{|c|}{ 178-1096C- } \\
\hline $2 \mathrm{H}$ & 168.24 & 2.056 & 10.05 & 3.275 & 0.099 & \\
\hline $2 \mathrm{H}$ & 169.90 & 2.074 & 10.35 & 2.925 & 0.043 & 8 \\
\hline $2 \mathrm{H}$ & 171.46 & 2.092 & 10.4 & 2.925 & 0.060 & 7.9 \\
\hline $2 \mathrm{H}$ & 173.03 & 2.110 & 10.6 & 3.075 & 0.073 & 7.9 \\
\hline $2 \mathrm{H}$ & 173.81 & 2.118 & 9.1 & 3.35 & 0.104 & \\
\hline $2 \mathrm{H}$ & 174.61 & 2.127 & 9.05 & 3.275 & 0.160 & \\
\hline \multicolumn{7}{|c|}{ 178-1096B- } \\
\hline $23 x$ & 174.70 & 2.128 & 10 & 3.325 & 0.068 & 8.1 \\
\hline \multicolumn{7}{|c|}{ 178-1096C- } \\
\hline $2 \mathrm{H}$ & 176.16 & 2.145 & 10.35 & 3.075 & 0.057 & 8.2 \\
\hline \multicolumn{7}{|c|}{ 178-1096B- } \\
\hline $23 x$ & 176.24 & 2.146 & 10.2 & 3.125 & 0.056 & \\
\hline \multicolumn{7}{|c|}{ 178-1096C- } \\
\hline $2 \mathrm{H}$ & 176.56 & 2.149 & 10.15 & 3.05 & 0.066 & \\
\hline \multicolumn{7}{|c|}{ 178-1096B- } \\
\hline $23 x$ & 177.64 & 2.161 & 10.2 & 3.05 & 0.033 & 8.1 \\
\hline $23 x$ & 179.15 & 2.178 & 10.6 & 3.15 & 0.032 & 8 \\
\hline $24 X$ & 184.09 & 2.234 & 10.45 & 3.125 & 0.072 & 8.1 \\
\hline $24 X$ & 185.50 & 2.250 & 10.45 & 3.425 & 0.095 & 8.1 \\
\hline $24 X$ & 187.00 & 2.267 & 10.05 & 3.025 & 0.074 & 7.9 \\
\hline $24 X$ & 188.50 & 2.283 & 9.85 & 3.15 & 0.095 & 8 \\
\hline $24 X$ & 190.00 & 2.300 & 9.2 & 3.35 & 0.164 & \\
\hline $24 x$ & 191.50 & 2.317 & 9.95 & 2.975 & 0.042 & \\
\hline $24 X$ & 192.42 & 2.327 & 9.7 & 3.2 & 0.125 & \\
\hline \multicolumn{7}{|c|}{ 178-1096C- } \\
\hline $3 x$ & 195.80 & 2.365 & 9.8 & 3.1 & 0.113 & 7.9 \\
\hline $3 x$ & 198.80 & 2.399 & 9.8 & 3.225 & 0.070 & 8.1 \\
\hline $3 x$ & 200.29 & 2.416 & 9.4 & 3.325 & -0.083 & 8.3 \\
\hline $3 x$ & 200.69 & 2.420 & 10.55 & 2.875 & 0.043 & 8.1 \\
\hline $3 x$ & 201.85 & 2.433 & 10.3 & 2.95 & 0.119 & 8 \\
\hline \multicolumn{7}{|c|}{ 178-1096B- } \\
\hline $26 X$ & 203.19 & 2.449 & 10.05 & 3.075 & 0.106 & 8.2 \\
\hline $26 X$ & 204.69 & 2.465 & 10.05 & 3.375 & 0.170 & 7.9 \\
\hline $26 x$ & 206.19 & 2.482 & 10.1 & 3.075 & 0.057 & 7.9 \\
\hline $26 x$ & 207.69 & 2.499 & 10.15 & 3.15 & 0.095 & 8.1 \\
\hline $26 X$ & 209.19 & 2.516 & 10.3 & 3.2 & 0.062 & 8.1 \\
\hline $26 X$ & 210.69 & 2.533 & 9.7 & 3.025 & 0.058 & \\
\hline $26 X$ & 211.94 & 2.547 & 10 & 3.25 & 0.092 & 8 \\
\hline $27 X$ & 213.70 & 2.567 & 10.2 & 3.15 & 0.063 & \\
\hline $27 X$ & 215.19 & 2.583 & 10.35 & 3.175 & 0.055 & 8 \\
\hline $27 x$ & 216.69 & 2.600 & 9.55 & 3.3 & 0.136 & \\
\hline $27 x$ & 217.59 & 2.590 & 10.5 & 3.3 & 0.061 & 8.1 \\
\hline $28 x$ & 223.29 & 2.618 & 9.6 & 3.45 & 0.101 & \\
\hline $28 X$ & 224.79 & 2.625 & 10.3 & 3.025 & 0.041 & 8.1 \\
\hline $28 X$ & 226.05 & 2.632 & 9.8 & 3.125 & 0.040 & \\
\hline $29 X$ & 232.29 & 2.663 & 10.2 & 3.25 & 0.108 & 7.9 \\
\hline $29 X$ & 233.79 & 2.670 & 9.75 & 3.45 & 0.174 & \\
\hline $29 X$ & 235.30 & 2.678 & 9.8 & 3.175 & 0.102 & 7.9 \\
\hline $29 X$ & 236.80 & 2.685 & 10.45 & 3.15 & 0.048 & 8 \\
\hline $29 x$ & 238.29 & 2.693 & 10.5 & 3.175 & 0.039 & 8.1 \\
\hline $30 x$ & 242.29 & 2.713 & 10.25 & 3.125 & 0.024 & \\
\hline $30 x$ & 243.79 & 2.720 & 10.25 & 2.975 & 0.025 & \\
\hline $30 x$ & 244.50 & 2.724 & 10.5 & 2.85 & 0.018 & \\
\hline $30 x$ & 245.10 & 2.727 & 10.3 & 3.1 & 0.081 & 7.9 \\
\hline $30 x$ & 245.69 & 2.729 & 10.55 & 2.9 & 0.034 & \\
\hline $30 x$ & 245.90 & 2.731 & 10.1 & 2.825 & 0.080 & 7.9 \\
\hline $30 x$ & 246.10 & 2.731 & 9 & 3.325 & -0.023 & \\
\hline $30 x$ & 246.29 & 2.732 & 9.7 & 2.95 & 0.068 & 8 \\
\hline $30 x$ & 246.49 & 2.733 & 10 & 2.825 & -0.009 & 8.1 \\
\hline $30 x$ & 246.70 & 2.734 & 10.15 & 2.975 & 0.092 & 8.1 \\
\hline $30 x$ & 246.87 & 2.735 & 10.4 & 3.1 & 0.032 & 8 \\
\hline $31 X$ & 248.70 & 2.744 & 10.55 & 3.175 & 0.039 & 7.9 \\
\hline $32 X$ & 251.39 & 2.758 & 10.25 & 3.325 & 0.038 & \\
\hline $32 X$ & 254.39 & 2.773 & 10.5 & 3.3 & 0.061 & 7.9 \\
\hline $32 X$ & 256.84 & 2.785 & 10.6 & 3.175 & 0.039 & \\
\hline
\end{tabular}


C.J. Pudsey

DATA Report: Grain-Size DATA

Table T7. Diatom percentage, Site 1101.

\begin{tabular}{rcc}
\hline $\begin{array}{c}\text { Depth } \\
\text { (mbsf) }\end{array}$ & $\begin{array}{c}\text { Age } \\
(\mathrm{Ma})\end{array}$ & $\begin{array}{c}\text { Diatoms } \\
(\%)\end{array}$ \\
\hline 0.00 & 0.000 & \\
9.01 & 0.128 & 8 \\
9.30 & 0.132 & 11 \\
9.60 & 0.136 & 6 \\
10.18 & 0.145 & 5 \\
10.80 & 0.154 & 7 \\
12.10 & 0.172 & 3 \\
13.60 & 0.193 & 4.5 \\
15.10 & 0.215 & 4 \\
16.60 & 0.236 & 5 \\
18.40 & 0.262 & 4 \\
19.80 & 0.282 & 8 \\
21.30 & 0.303 & 3 \\
22.80 & 0.324 & 15 \\
23.40 & 0.333 & 11 \\
24.29 & 0.345 & 2 \\
25.80 & 0.367 & 3 \\
27.85 & 0.396 & 3 \\
29.35 & 0.417 & 5 \\
30.85 & 0.439 & 0 \\
32.35 & 0.460 & 1 \\
33.85 & 0.481 & 0.5 \\
35.35 & 0.503 & 6 \\
36.85 & 0.524 & 3 \\
37.10 & 0.528 & 5 \\
37.50 & 0.533 & 8 \\
39.80 & 0.566 & 0.5 \\
41.30 & 0.587 & 1 \\
42.80 & 0.609 & 4 \\
44.30 & 0.630 & 0.5 \\
45.80 & 0.651 & 0 \\
47.30 & 0.673 & 2 \\
47.79 & 0.680 & 1 \\
49.29 & 0.701 & 6 \\
49.29 & 0.701 & 6 \\
50.79 & 0.722 & 2 \\
52.29 & 0.743 & 0.5 \\
53.53 & 0.761 & 2 \\
53.79 & 0.765 & 2 \\
54.29 & 0.772 & 0.5 \\
55.29 & 0.786 & 2 \\
56.79 & 0.805 & 4 \\
57.26 & 0.811 & 5 \\
57.29 & 0.811 & 1 \\
58.79 & 0.831 & 2 \\
60.25 & 0.849 & 5 \\
61.86 & 0.870 & 2 \\
63.30 & 0.889 & 0.5 \\
64.79 & 0.908 & 1 \\
66.29 & 0.927 & 3 \\
66.80 & 0.934 & 2 \\
68.30 & 0.953 & 4 \\
69.80 & 0.972 & 1 \\
& &
\end{tabular}

\begin{tabular}{ccc}
\hline $\begin{array}{c}\text { Depth } \\
\text { (mbsf) }\end{array}$ & $\begin{array}{c}\text { Age } \\
(\mathrm{Ma})\end{array}$ & $\begin{array}{c}\text { Diatoms } \\
(\%)\end{array}$ \\
\hline 70.64 & 0.983 & 4 \\
71.30 & 0.991 & 3 \\
72.04 & 1.001 & 2 \\
72.80 & 1.011 & 0 \\
74.30 & 1.030 & 3 \\
75.50 & 1.045 & 9 \\
76.33 & 1.056 & 5 \\
76.80 & 1.080 & 4 \\
77.80 & 1.096 & 0.5 \\
79.30 & 1.119 & 5 \\
80.10 & 1.132 & 6 \\
80.80 & 1.143 & 4 \\
81.15 & 1.148 & 7 \\
82.30 & 1.166 & 4 \\
83.84 & 1.190 & 3 \\
84.90 & 1.207 & 7 \\
85.79 & 1.221 & 1 \\
87.30 & 1.244 & 1 \\
88.80 & 1.268 & 1 \\
90.30 & 1.291 & 2 \\
91.84 & 1.315 & 2 \\
92.90 & 1.332 & 3 \\
93.28 & 1.338 & 4 \\
94.10 & 1.351 & 2 \\
95.56 & 1.374 & 3 \\
96.80 & 1.393 & 4 \\
97.10 & 1.398 & 2 \\
97.70 & 1.407 & 5 \\
98.30 & 1.417 & 5 \\
98.79 & 1.424 & 2 \\
99.20 & 1.431 & 4 \\
99.79 & 1.440 & 4 \\
101.32 & 1.464 & 3 \\
102.84 & 1.488 & 0 \\
104.29 & 1.510 & 0 \\
104.84 & 1.519 & 0.5 \\
106.29 & 1.542 & 0 \\
107.79 & 1.565 & 1 \\
109.29 & 1.589 & 0 \\
110.50 & 1.607 & 3 \\
110.79 & 1.612 & 2 \\
111.10 & 1.617 & 3 \\
112.29 & 1.635 & 0.5 \\
113.29 & 1.651 & 0 \\
115.85 & 1.691 & 3 \\
116.58 & 1.703 & 0 \\
116.62 & 1.703 & 0 \\
116.66 & 1.704 & 0.5 \\
116.70 & 1.704 & 0 \\
116.75 & 1.705 & 0 \\
117.28 & 1.714 & 0 \\
118.80 & 1.737 & 2 \\
120.31 & 1.761 & 0 \\
& &
\end{tabular}

\begin{tabular}{|c|c|c|}
\hline $\begin{array}{l}\text { Depth } \\
\text { (mbsf) }\end{array}$ & $\begin{array}{l}\text { Age } \\
(\mathrm{Ma})\end{array}$ & $\begin{array}{l}\text { Diatoms } \\
\text { (\%) }\end{array}$ \\
\hline 121.20 & 1.775 & 2 \\
\hline 121.80 & 1.794 & 0 \\
\hline 123.30 & 1.839 & 4 \\
\hline 123.86 & 1.856 & 3 \\
\hline 123.80 & 1.854 & 1 \\
\hline 125.30 & 1.899 & 4.5 \\
\hline 126.80 & 1.944 & 3 \\
\hline 128.30 & 1.970 & 2 \\
\hline 128.43 & 1.972 & 5 \\
\hline 129.87 & 1.996 & 2 \\
\hline 131.37 & 2.020 & 0 \\
\hline 132.87 & 2.044 & 0 \\
\hline 133.30 & 2.051 & 2 \\
\hline 134.80 & 2.075 & 1 \\
\hline 136.30 & 2.100 & 4 \\
\hline 137.30 & 2.116 & 3 \\
\hline 137.90 & 2.125 & 6.5 \\
\hline 138.49 & 2.135 & 3 \\
\hline 139.99 & 2.159 & 6 \\
\hline 142.79 & 2.204 & 10 \\
\hline 150.60 & 2.331 & 5.5 \\
\hline 160.19 & 2.485 & 3 \\
\hline 169.79 & 2.641 & 8 \\
\hline 179.43 & 2.721 & 11.5 \\
\hline 188.99 & 2.822 & 12 \\
\hline 190.03 & 2.833 & 16 \\
\hline 190.50 & 2.838 & 16.5 \\
\hline 192.00 & 2.854 & 33 \\
\hline 193.06 & 2.865 & 17 \\
\hline 193.50 & 2.870 & 27 \\
\hline 194.43 & 2.880 & 25 \\
\hline 194.97 & 2.885 & 27 \\
\hline 195.84 & 2.895 & 21 \\
\hline 196.50 & 2.902 & 25 \\
\hline 197.50 & 2.912 & 11 \\
\hline 198.00 & 2.917 & 10 \\
\hline 198.70 & 2.925 & 9 \\
\hline 200.08 & 2.939 & 29 \\
\hline 201.58 & 2.955 & 32 \\
\hline 202.48 & 2.965 & 21 \\
\hline 203.12 & 2.972 & 28 \\
\hline 204.10 & 2.982 & 23 \\
\hline 204.59 & 2.987 & 22 \\
\hline 206.07 & 3.003 & 21 \\
\hline 207.57 & 3.019 & 24 \\
\hline 208.23 & 3.026 & 30 \\
\hline 209.11 & 3.035 & 37 \\
\hline 209.71 & 3.041 & 37 \\
\hline 211.18 & 3.057 & 25 \\
\hline 212.68 & 3.073 & 30 \\
\hline 214.22 & 3.089 & 27 \\
\hline 215.73 & 3.105 & 21 \\
\hline 217.21 & 3.121 & 26 \\
\hline
\end{tabular}


C.J. Pudsey

DATA Report: Grain-Size DATA

Table T8. Fine-fraction $(>4 \phi)$ size distribution (percentage finer than each 1- $\phi$ interval), Site 1101. (Continued on next two pages.)

\begin{tabular}{|c|c|c|c|c|c|c|c|c|c|c|}
\hline \multirow[b]{2}{*}{ Hole } & \multirow{2}{*}{$\begin{array}{l}\text { Depth } \\
\text { (mbsf) }\end{array}$} & \multirow{2}{*}{$\begin{array}{l}\text { Age } \\
\text { (Ma) }\end{array}$} & \multicolumn{8}{|c|}{ Grain size $(\phi)$} \\
\hline & & & 4 & 5 & 6 & 7 & 8 & 9 & 10 & 11 \\
\hline \multicolumn{11}{|c|}{$178-1101 \mathrm{~A}-$} \\
\hline $2 \mathrm{H}$ & 9.01 & 0.128 & 98.50 & 97.50 & 89.81 & 75.92 & 61.64 & 50.45 & 42.26 & 34.07 \\
\hline $2 \mathrm{H}$ & 9.30 & 0.132 & 98.20 & 97.79 & 95.00 & 81.87 & 64.50 & 50.03 & 39.80 & 29.77 \\
\hline $2 \mathrm{H}$ & 9.60 & 0.136 & 98.60 & 97.99 & 97.18 & 92.72 & 78.62 & 62.28 & 50.11 & 38.75 \\
\hline $2 \mathrm{H}$ & 10.18 & 0.145 & 97.80 & 95.16 & 86.54 & 71.07 & 57.07 & 46.70 & 38.96 & 31.13 \\
\hline $2 \mathrm{H}$ & 10.80 & 0.154 & 95.90 & 94.64 & 88.13 & 75.23 & 61.54 & 50.96 & 42.81 & 34.17 \\
\hline $2 \mathrm{H}$ & 12.10 & 0.172 & 98.70 & 98.70 & 96.33 & 87.15 & 71.47 & 57.86 & 48.47 & 38.88 \\
\hline $2 \mathrm{H}$ & 13.60 & 0.193 & 99.40 & 99.09 & 96.36 & 85.78 & 68.40 & 54.36 & 44.52 & 35.40 \\
\hline $2 \mathrm{H}$ & 15.10 & 0.215 & 99.90 & 99.90 & 98.34 & 91.07 & 77.68 & 63.87 & 53.58 & 43.72 \\
\hline $2 \mathrm{H}$ & 16.60 & 0.236 & 98.00 & 98.00 & 96.23 & 85.09 & 67.28 & 53.53 & 44.57 & 34.78 \\
\hline $3 \mathrm{H}$ & 18.40 & 0.262 & 99.30 & 99.30 & 97.53 & 89.29 & 73.22 & 59.45 & 49.13 & 39.01 \\
\hline $3 \mathrm{H}$ & 19.80 & 0.282 & 97.00 & 90.50 & 70.02 & 49.63 & 36.04 & 28.26 & 22.45 & 16.94 \\
\hline $3 \mathrm{H}$ & 21.30 & 0.303 & 97.00 & 97.00 & 90.67 & 76.73 & 60.27 & 48.13 & 38.95 & 29.87 \\
\hline $3 \mathrm{H}$ & 22.80 & 0.324 & 97.40 & 97.18 & 89.50 & 72.29 & 54.44 & 42.53 & 32.90 & 23.59 \\
\hline $3 \mathrm{H}$ & 23.40 & 0.333 & 99.40 & 99.40 & 99.09 & 91.80 & 76.29 & 60.58 & 49.34 & 38.51 \\
\hline $3 \mathrm{H}$ & 24.29 & 0.345 & 99.10 & 99.10 & 96.09 & 86.13 & 71.19 & 58.11 & 48.67 & 38.50 \\
\hline $3 \mathrm{H}$ & 25.80 & 0.367 & 99.90 & 99.90 & 95.34 & 86.32 & 73.86 & 61.80 & 52.28 & 43.06 \\
\hline $4 \mathrm{H}$ & 27.85 & 0.396 & 99.90 & 99.59 & 98.33 & 87.94 & 72.41 & 58.97 & 48.59 & 39.14 \\
\hline $4 \mathrm{H}$ & & 0.417 & 99.50 & 99.50 & 97.72 & & 74.13 & & & 42.67 \\
\hline $4 \mathrm{H}$ & 30.85 & 0.439 & 99.50 & 98.79 & 90.68 & 78.10 & 64.41 & 53.45 & 45.03 & 37.02 \\
\hline $4 \mathrm{H}$ & 32.35 & 0.460 & 99.90 & 99.90 & 98.53 & 88.98 & 72.90 & 59.67 & & 40.65 \\
\hline $4 \mathrm{H}$ & 33.85 & 0.481 & 99.90 & 98.48 & 89.87 & 76.60 & 63.73 & 52.89 & 44.99 & 37.79 \\
\hline $4 \mathrm{H}$ & 35.35 & 0.503 & 97.70 & 97.01 & 91.25 & 77.37 & 60.01 & 45.33 & & \\
\hline $4 \mathrm{H}$ & 36.85 & 0.524 & 98.30 & 97.86 & 90.46 & 72.83 & 50.29 & 33.75 & 23.84 & 14.48 \\
\hline $4 \mathrm{H}$ & 37.10 & 0.528 & 98.10 & 87.62 & 65.77 & 47.20 & 35.03 & 26.45 & 19.56 & 12.97 \\
\hline $4 \mathrm{H}$ & 37.50 & 0.533 & 98.10 & 96.51 & 87.00 & 67.97 & 48.94 & 36.48 & 27.07 & 17.78 \\
\hline $6 \mathrm{H}$ & 39.80 & 0.566 & 99.90 & 99.90 & 98.65 & 90.62 & 77.06 & 59.34 & 44.94 & 38.27 \\
\hline $6 \mathrm{H}$ & 41.30 & 0.587 & 99.90 & 99.69 & 97.39 & 89.54 & 76.57 & 63.60 & 54.19 & 44.77 \\
\hline $6 \mathrm{H}$ & 42.80 & 0.609 & 98.60 & 97.90 & 89.79 & 74.98 & 59.56 & 48.95 & 41.24 & 33.43 \\
\hline $6 \mathrm{H}$ & 44.30 & 0.630 & 99.80 & 98.66 & 97.63 & 90.59 & 77.13 & 64.19 & 54.35 & 44.83 \\
\hline $6 \mathrm{H}$ & 45.80 & 0.651 & 99.90 & 98.83 & 95.18 & 85.84 & 72.22 & 59.98 & 50.11 & 40.88 \\
\hline $6 \mathrm{H}$ & & & & & & & & & & \\
\hline $7 \mathrm{H}$ & 47.79 & 0.680 & 99.40 & 98.07 & 87.80 & 71.67 & 55.76 & 43.95 & 35.12 & 26.70 \\
\hline $7 \mathrm{H}$ & & & & & & & & & & \\
\hline $7 \mathrm{H}$ & 50.79 & 0.701 & 99.60 & 98.59 & 89.97 & 73.76 & 57.25 & 44.58 & 35.36 & 25.94 \\
\hline $7 \mathrm{H}$ & 52.29 & 0.722 & 100.00 & & & & & & & 36.57 \\
\hline $7 \mathrm{H}$ & 53.53 & 0.743 & 91.30 & 88.73 & 78.70 & 61.14 & 45.70 & 34.75 & 27.12 & 20.32 \\
\hline $7 \mathrm{H}$ & 53.79 & 0.765 & 95.90 & 94.15 & 84.41 & 64.36 & 44.40 & 30.67 & 21.22 & 13.63 \\
\hline $7 \mathrm{H}$ & 54.29 & 0.772 & 100.00 & 100.00 & 99.27 & 92.40 & 78.77 & 64.41 & 54.11 & 43.70 \\
\hline $7 \mathrm{H}$ & 55.29 & 0.786 & 98.00 & 94.32 & 82.79 & 60.53 & 40.65 & 26.64 & 18.09 & 11.03 \\
\hline $7 \mathrm{H}$ & 56.79 & 0.805 & 80.80 & 76.72 & 61.14 & 41.57 & 27.49 & 18.83 & 13.74 & 10.33 \\
\hline $7 \mathrm{H}$ & 57.26 & 0.811 & 99.20 & 98.70 & 94.68 & 81.11 & 62.62 & 45.93 & 34.67 & 25.33 \\
\hline $8 \mathrm{H}$ & 57.29 & 0.811 & 91.30 & 88.97 & 80.13 & 65.05 & 49.05 & 37.13 & 28.20 & 20.48 \\
\hline $8 \mathrm{H}$ & 58.79 & 0.831 & 100.00 & 98.89 & 94.84 & 82.61 & 68.86 & 58.04 & 49.44 & 42.26 \\
\hline $8 \mathrm{H}$ & & 0.849 & 99.00 & 94.67 & 81.29 & & & & & 16.80 \\
\hline $8 \mathrm{H}$ & 61.86 & 0.870 & 99.10 & 97.99 & 90.33 & 76.92 & 64.12 & 53.03 & 44.86 & 36.49 \\
\hline $8 \mathrm{H}$ & & & & & & & & & & 35.66 \\
\hline $8 \mathrm{H}$ & 64.79 & 0.908 & 98.70 & 97.69 & 89.55 & 74.58 & 60.61 & 49.65 & 42.01 & 34.88 \\
\hline $8 \mathrm{H}$ & & & & & & & & & & 25.11 \\
\hline $9 \mathrm{H}$ & 66.80 & 0.934 & 99.90 & 99.29 & 94.18 & 81.82 & 64.86 & 50.26 & 39.12 & 29.83 \\
\hline $9 \mathrm{H}$ & 68.30 & 0.953 & 99.50 & 95.43 & 76.24 & 53.61 & 34.94 & 24.30 & 16.79 & 1.36 \\
\hline $9 \mathrm{H}$ & 69.80 & 0.972 & 99.80 & 99.70 & 96.34 & 85.66 & 71.82 & 58.70 & 48.63 & 38.35 \\
\hline $9 \mathrm{H}$ & 70.64 & 0.983 & 95.10 & 90.83 & 74.94 & 52.64 & 35.67 & 25.59 & 18.71 & 13.09 \\
\hline $9 \mathrm{H}$ & 71.30 & 0.991 & 99.80 & 97.54 & 82.43 & 55.81 & 34.74 & 22.71 & 15.11 & 9.46 \\
\hline $9 \mathrm{H}$ & 72.04 & 1.001 & 92.30 & 88.39 & 72.37 & 52.63 & 35.66 & 24.12 & 16.11 & 9.63 \\
\hline $9 \mathrm{H}$ & 72.80 & 1.011 & 100.00 & 99.80 & 95.83 & 84.13 & 70.19 & 58.09 & 49.14 & 41.00 \\
\hline $9 \mathrm{H}$ & 74.30 & 1.030 & 88.80 & 86.49 & 81.68 & 73.76 & 62.73 & 52.14 & 44.04 & 36.30 \\
\hline $9 \mathrm{H}$ & 75.50 & 1.045 & 98.10 & 91.52 & 69.96 & & & & & 15.69 \\
\hline $10 \mathrm{H}$ & 76.33 & 1.056 & 91.50 & 86.27 & 73.24 & 51.94 & 33.68 & 22.21 & 14.41 & 8.99 \\
\hline $10 \mathrm{H}$ & 76.80 & 1.080 & 99.90 & 98.80 & 92.88 & 81.65 & 67.30 & 55.27 & 45.54 & 35.51 \\
\hline $10 \mathrm{H}$ & 77.80 & 1.096 & 99.90 & 98.49 & 92.63 & 80.22 & 65.39 & 54.39 & 45.71 & 37.54 \\
\hline $10 \mathrm{H}$ & 79.30 & 1.119 & 99.60 & 97.24 & 83.82 & 62.10 & 43.04 & 31.25 & 23.26 & 15.47 \\
\hline $10 \mathrm{H}$ & 80.10 & 1.132 & 91.70 & 87.04 & 70.55 & 50.32 & 33.36 & 22.37 & 14.72 & 8.29 \\
\hline $10 \mathrm{H}$ & 80.80 & 1.143 & 81.70 & 78.55 & 67.20 & 49.30 & 33.89 & 23.45 & 16.65 & 10.03 \\
\hline $10 \mathrm{H}$ & 81.15 & 1.148 & 99.50 & 96.36 & 83.41 & 61.14 & 41.60 & 28.24 & 19.64 & 12.05 \\
\hline $10 \mathrm{H}$ & 82.30 & 1.166 & 97.10 & 96.02 & 89.46 & 76.82 & 62.81 & 51.54 & 42.92 & 35.08 \\
\hline $10 \mathrm{H}$ & 83.84 & 1.190 & 99.90 & 99.19 & 94.84 & 83.81 & 70.14 & 59.11 & 50.30 & 41.60 \\
\hline
\end{tabular}


C.J. Pudsey

DATA Report: Grain-Size DATA

Table T8 (continued).

\begin{tabular}{|c|c|c|c|c|c|c|c|c|c|c|}
\hline \multirow[b]{2}{*}{ Hole } & \multirow{2}{*}{$\begin{array}{l}\text { Depth } \\
\text { (mbsf) }\end{array}$} & \multirow{2}{*}{$\begin{array}{c}\text { Age } \\
(\mathrm{Ma})\end{array}$} & \multicolumn{8}{|c|}{ Grain size $(\phi)$} \\
\hline & & & 4 & 5 & 6 & 7 & 8 & 9 & 10 & 11 \\
\hline $10 \mathrm{H}$ & 84.90 & 207 & 92.90 & 85.53 & 64.77 & 46.50 & 34.06 & 74 & 19.52 & 13.20 \\
\hline $11 \mathrm{H}$ & .79 & 221 & 99.50 & 99.50 & 96.94 & 90.58 & 77.06 & 63.33 & 53.49 & 42.32 \\
\hline $11 \mathrm{H}$ & 87.30 & 244 & 99.70 & 98.86 & 96.45 & 85.22 & 68.95 & 56.57 & 47.86 & 39.25 \\
\hline $11 \mathrm{H}$ & 8 & & 99.80 & 98.97 & & 1 & & & & \\
\hline $11 \mathrm{H}$ & 90.30 & 291 & 99.90 & 99.08 & 97.94 & .34 & 77.43 & & & 4.43 \\
\hline $11 \mathrm{H}$ & 91.84 & .315 & 98.20 & 97.66 & 91.57 & 74.49 & 55.14 & 40.45 & 9.80 & 19.68 \\
\hline $11 \mathrm{H}$ & 92.90 & 1.332 & 98.60 & 98.50 & 95.80 & 86.15 & 69.12 & 53.76 & 41.72 & 30.62 \\
\hline $11 \mathrm{H}$ & 93.28 & & 100.00 & 100.00 & & 88.35 & & & & \\
\hline $11 \mathrm{H}$ & 9 & & 89.20 & & & 2 & & & & \\
\hline $12 \mathrm{H}$ & 95.56 & .374 & 100.00 & 99.79 & 98.23 & 89.81 & & & & \\
\hline $12 \mathrm{H}$ & 96.80 & 1.393 & 99.90 & & 99.07 & & & & & \\
\hline $12 \mathrm{H}$ & 97.10 & 1.398 & & 79.30 & 67.67 & 50.73 & & & & 14.33 \\
\hline $12 \mathrm{H}$ & 97 & & & 98. & & & & & & \\
\hline $12 \mathrm{H}$ & 98.30 & 1 & 99.60 & 99.60 & 9 & 1 & 60 & & & \\
\hline $12 \mathrm{H}$ & 98.79 & 1.4 & 92.80 & 88.20 & 74.95 & 58.05 & & & & \\
\hline $12 \mathrm{H}$ & 99.20 & 1. & 99.30 & 98 & 92 & & & & & \\
\hline $12 \mathrm{H}$ & 99.79 & & & 97.49 & 92.47 & 79.62 & & & & \\
\hline $12 \mathrm{H}$ & & & & & & & & & & \\
\hline $12 \mathrm{H}$ & 2. & 1 & 99.80 & 99.49 & & & & & & \\
\hline $12 \mathrm{H}$ & 104.2 & 1 & 100.00 & 100.00 & & 02 & & & & \\
\hline $13 \mathrm{H}$ & 104.84 & 1.5 & 99.20 & & 92.42 & 8016 & & & & \\
\hline $13 \mathrm{H}$ & & & & & & & & & & \\
\hline $13 \mathrm{H}$ & & & & & & & & & & \\
\hline $13 \mathrm{H}$ & & & & & & & & & & \\
\hline $13 \mathrm{H}$ & 11 & 1 & 96 & & & & & & & \\
\hline $13 \mathrm{H}$ & 110.79 & 1.612 & 88.90 & 84.54 & 71.08 & 49.44 & 31.36 & 04 & 27 & 64 \\
\hline $13 \mathrm{H}$ & & & & & & & & & & \\
\hline $13 \mathrm{H}$ & 112. & 1 & 00.00 & 98. & 95 & 3 & & & & \\
\hline $13 \mathrm{H}$ & & & & & & & & & & \\
\hline $14 \mathrm{H}$ & 1 & 1.69 & 9 & 97 & 88 & 0 & & & & \\
\hline $14 \mathrm{H}$ & 28 & 17 & 99.90 & & & & & & & 39.94 \\
\hline $14 \mathrm{H}$ & & & & & & & & & & \\
\hline $14 \mathrm{H}$ & 120 & & 98.20 & 97.70 & & 0 & & & & \\
\hline $14 \mathrm{H}$ & & & 90 & 93. & 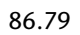 & & & & & \\
\hline $14 \mathrm{H}$ & 121.80 & 1.794 & 99.80 & 99.20 & 95.30 & 87.70 & 73.70 & 60.20 & 49.20 & 38.20 \\
\hline $14 \mathrm{H}$ & 12330 & & & & & & & & & \\
\hline $14 \mathrm{H}$ & & & & & & & & & & \\
\hline $15 \mathrm{H}$ & & & & & & & & & & \\
\hline $15 \mathrm{H}$ & & & & & & & & & & \\
\hline $15 \mathrm{H}$ & 126.80 & 1044 & 98.40 & 96.40 & 84.33 & 64.37 & 47.10 & 34.93 & 5.95 & 18.26 \\
\hline $15 \mathrm{H}$ & 1282 & & & & & & & & & \\
\hline $15 \mathrm{H}$ & & & & & & & & & & \\
\hline $15 \mathrm{H}$ & & & 0 & 98. & 1 & 8 & & & & \\
\hline $15 \mathrm{H}$ & 13 & 2. & 99.50 & 98.60 & 91.40 & 79.10 & & 2.00 & 0 & 35.70 \\
\hline $15 \mathrm{H}$ & & & & & 95.59 & & & & & 42.63 \\
\hline $16 \mathrm{H}$ & & & & 97 & & & & & & \\
\hline $16 \mathrm{H}$ & & & & & & & & & & 60 \\
\hline $16 \mathrm{H}$ & 13 & & 00.00 & 100 & 6 & & & 4 & & 95 \\
\hline $16 \mathrm{H}$ & & & & & 97.03 & & & & & 42.26 \\
\hline $16 \mathrm{H}$ & & & & & & & & & & \\
\hline $16 \mathrm{H}$ & & & & & & & & & & \\
\hline $16 \mathrm{H}$ & 1 & & 0 & 04 & 77. & 5 & 45 & 36. & 6 & 27 \\
\hline $17 X$ & & & .80 & & & 7 & & 47.90 & & 70 \\
\hline $18 \mathrm{X}$ & 150.60 & 2.331 & 98.80 & 97.18 & 84.86 & 65.66 & 48.89 & 37.98 & 9.50 & 21.92 \\
\hline $19 \mathrm{X}$ & & & & & & 60.95 & & & & 19.88 \\
\hline $20 x$ & & & & & & 109 & 71.82 & & & 270 \\
\hline $21 x$ & 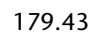 & & & & 8 & 2 & & 5 & 0 & 2 \\
\hline $22 x$ & 1 & & 99.10 & 97.71 & 91 & 4 & & & & 40.37 \\
\hline $22 x$ & 190.03 & 2.833 & 95.40 & 95.01 & 93.34 & 86.86 & 75.18 & 63.11 & 3.10 & 42.79 \\
\hline $22 x$ & & & 5.80 & & 89.39 & 78.40 & & & & 34.23 \\
\hline $22 X$ & & & & & & & & & & ר \\
\hline $22 x$ & & & 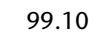 & 0640 & 8630 & 1070 & 54.30 & 44.10 & 5.90 & 2600 \\
\hline $22 x$ & 193.50 & 2.870 & 98.70 & 98.40 & 93.36 & 79.77 & 65.16 & 53.88 & 45.02 & 36.26 \\
\hline $22 X$ & 194.43 & 2.880 & 98.70 & 93.60 & 77.60 & 58.90 & 43.80 & 32.80 & 24.90 & 18.10 \\
\hline $22 x$ & & 2.88 & & 93.57 & & & & & & 17.5 \\
\hline 2 & & & & & & & & & & \\
\hline $22 x$ & & & & & & 68 & & 39 & 28.99 & 18.28 \\
\hline $22 x$ & 197.50 & 2.912 & 99.30 & 98.60 & 94.72 & 84.28 & 71.74 & 59.70 & 49.85 & 40.20 \\
\hline $22 x$ & 198.00 & 2.917 & 99.25 & 97.76 & 93.08 & 83.22 & 69.48 & 56.84 & 47.09 & 39.12 \\
\hline
\end{tabular}


C.J. Pudsey

DATA Report: Grain-Size DATA

Table T8 (continued).

\begin{tabular}{|c|c|c|c|c|c|c|c|c|c|c|}
\hline \multirow[b]{2}{*}{ Hole } & \multirow{2}{*}{$\begin{array}{l}\text { Depth } \\
\text { (mbsf) }\end{array}$} & \multirow{2}{*}{$\begin{array}{l}\text { Age } \\
(\mathrm{Ma})\end{array}$} & \multicolumn{8}{|c|}{ Grain size $(\phi)$} \\
\hline & & & 4 & 5 & 6 & 7 & 8 & 9 & 10 & 11 \\
\hline $23 x$ & 198.70 & 2.925 & 99.80 & 95.80 & 81.50 & 66.60 & 53.10 & 42.40 & 34.00 & 27.10 \\
\hline $23 x$ & 200.08 & 2.939 & 99.50 & 96.21 & 84.63 & 69.56 & 55.69 & 44.91 & 35.63 & 27.05 \\
\hline $23 x$ & 201.58 & 2.955 & 98.60 & 98.20 & 93.81 & 82.03 & 67.66 & 55.59 & 46.51 & 38.32 \\
\hline $23 x$ & 202.48 & 2.965 & 99.00 & 96.21 & 86.92 & 72.65 & 57.78 & 46.31 & 37.32 & 29.54 \\
\hline $23 x$ & 203.12 & 2.972 & 99.80 & 97.49 & 85.72 & 68.91 & 54.63 & 43.76 & 35.71 & 28.47 \\
\hline $23 x$ & 204.10 & 2.982 & 95.50 & 94.16 & 88.05 & 76.30 & 61.98 & 51.09 & 42.98 & 34.67 \\
\hline $23 x$ & 204.59 & 2.987 & 99.50 & 98.70 & 96.12 & 87.97 & 75.74 & 62.13 & 51.39 & 41.15 \\
\hline $23 x$ & 206.07 & 3.003 & 99.30 & 97.42 & 94.25 & 85.74 & 73.66 & 60.99 & 50.39 & 39.30 \\
\hline $23 x$ & 207.57 & 3.019 & 99.80 & 97.38 & 81.25 & 65.12 & 53.93 & 45.06 & 36.59 & 27.62 \\
\hline $24 X$ & 208.23 & 3.026 & 100.00 & 99.79 & 99.59 & 91.62 & 77.87 & 63.60 & 51.91 & 40.54 \\
\hline $24 X$ & 209.11 & 3.035 & 99.80 & 99.20 & 96.69 & 86.76 & 72.62 & 59.58 & 48.45 & 37.41 \\
\hline $24 X$ & 209.71 & 3.041 & 99.60 & 99.60 & 97.85 & 87.25 & 71.00 & 56.59 & 46.61 & 35.40 \\
\hline $24 X$ & 211.18 & 3.057 & 99.40 & 99.40 & 97.23 & 87.00 & 70.57 & 55.59 & 44.12 & 32.24 \\
\hline $24 X$ & 212.68 & 3.073 & 99.50 & 98.30 & 91.68 & 78.84 & 63.89 & 51.86 & 42.33 & 33.00 \\
\hline $24 X$ & 214.22 & 3.089 & 99.20 & 98.66 & 93.92 & 79.04 & 63.19 & 50.46 & 40.87 & 30.73 \\
\hline $24 X$ & 215.73 & 3.105 & 99.60 & 97.39 & 88.95 & 72.16 & 56.58 & 45.23 & 36.48 & 27.04 \\
\hline $24 X$ & 217.21 & 3.121 & 99.80 & 99.80 & 98.66 & 90.82 & 76.17 & 62.44 & 51.09 & 39.63 \\
\hline
\end{tabular}


C.J. Pudsey

DAta Report: Grain-Size Data

Table T9. Median diameter, mode, sorting, and skewness, Site 1101. (See table note. Continued on next page.)

\begin{tabular}{|c|c|c|c|c|c|}
\hline $\begin{array}{l}\text { Depth } \\
\text { (mbsf) }\end{array}$ & $\begin{array}{l}\text { Age } \\
(\mathrm{Ma})\end{array}$ & $\phi 50$ & Sorting & Skewness & $\begin{array}{c}\text { Mode } \\
(\phi)\end{array}$ \\
\hline 9.01 & 0.128 & 9 & 2.8 & 0.232 & 7.2 \\
\hline 9.30 & 0.132 & 9.05 & 3.425 & 0.226 & 7.7 \\
\hline 9.60 & 0.136 & 10 & 2.675 & 0.121 & 8.2 \\
\hline 10.18 & 0.145 & 8.65 & 3.375 & 0.274 & 6.6 \\
\hline 10.80 & 0.154 & 9.1 & 3.475 & 0.209 & 7 \\
\hline 12.10 & 0.172 & 9.8 & 3.15 & 0.175 & 7.8 \\
\hline 13.60 & 0.193 & 9.4 & 3.05 & 0.246 & 7.9 \\
\hline 15.10 & 0.215 & 10.4 & 3.075 & 0.089 & 7.9 \\
\hline 16.60 & 0.236 & 9.4 & 3 & 0.217 & 7.8 \\
\hline 18.40 & 0.262 & 9.9 & 2.925 & 0.128 & 7.6 \\
\hline 19.80 & 0.282 & 7 & 2.9 & 0.431 & 6 \\
\hline 21.30 & 0.303 & 8.8 & 3.05 & 0.246 & 7.8 \\
\hline 22.80 & 0.324 & 8.3 & 2.775 & 0.279 & 7 \\
\hline 23.40 & 0.333 & 9.95 & 2.725 & 0.119 & 7.9 \\
\hline 24.29 & 0.345 & 9.85 & 3.075 & 0.122 & 8 \\
\hline 25.80 & 0.367 & 10.25 & 3.35 & 0.090 & 8 \\
\hline 27.85 & 0.396 & 9.85 & 3.125 & 0.168 & 7.9 \\
\hline 29.35 & 0.417 & 10.2 & 3.25 & 0.123 & 8 \\
\hline 30.85 & 0.439 & 9.4 & 3.525 & 0.191 & 7.2 \\
\hline 32.35 & 0.460 & 10 & 3.125 & 0.152 & 8.9 \\
\hline 33.85 & 0.481 & 9.4 & 3.8 & 0.211 & \\
\hline 35.35 & 0.503 & 8.65 & 2.6 & 0.212 & 7.8 \\
\hline 36.85 & 0.524 & 8 & 2.225 & 0.281 & 8 \\
\hline 37.10 & 0.528 & 6.8 & 2.675 & 0.402 & 5.8 \\
\hline 37.50 & 0.533 & 7.95 & 2.525 & 0.287 & \\
\hline 39.80 & 0.566 & 9.5 & 3.425 & 0.431 & \\
\hline 41.30 & 0.587 & 10.45 & 3.35 & 0.119 & 7.9 \\
\hline 42.80 & 0.609 & 8.85 & 3.475 & 0.281 & 7.4 \\
\hline 44.30 & 0.630 & 10.45 & 3.225 & 0.101 & 8 \\
\hline 45.80 & 0.651 & 10 & 3.275 & 0.145 & 8 \\
\hline 47.30 & 0.673 & 9.4 & 2.875 & 0.183 & 8 \\
\hline 47.79 & 0.680 & 8.4 & 3 & 0.283 & \\
\hline 49.29 & 0.701 & 7.7 & 2.55 & 0.333 & 6.6 \\
\hline 50.79 & 0.701 & 8.5 & 2.875 & 0.252 & \\
\hline 52.29 & 0.722 & 9 & 3.8 & 0.368 & \\
\hline 53.53 & 0.743 & 7.7 & 3.075 & 0.301 & 6.9 \\
\hline 53.79 & 0.765 & 7.7 & 2.35 & 0.277 & 6.6 \\
\hline 54.29 & 0.772 & 10.45 & 3.025 & 0.074 & 8.2 \\
\hline 55.29 & 0.786 & 7.5 & 2.2 & 0.273 & 6.9 \\
\hline 56.79 & 0.805 & 6.55 & 3.275 & -0.084 & 6.2 \\
\hline 57.26 & 0.811 & 8.7 & 2.65 & 0.283 & 7.8 \\
\hline 57.29 & 0.811 & 7.95 & 3 & 0.233 & 7.2 \\
\hline 58.79 & 0.831 & 9.95 & 3.95 & 0.228 & \\
\hline 60.25 & 0.849 & 7.75 & 2.65 & 0.283 & 6.5 \\
\hline 61.86 & 0.870 & 9.35 & 3.5 & 0.186 & \\
\hline 63.30 & 0.889 & 9.35 & 3.3 & 0.167 & 7.8 \\
\hline 64.79 & 0.908 & 8.95 & 3.6 & 0.292 & 6.6 \\
\hline 66.29 & 0.927 & 8.2 & 3.1 & 0.323 & 6.9 \\
\hline 66.80 & 0.934 & 9 & 2.875 & 0.235 & 7.9 \\
\hline 68.30 & 0.953 & 7.2 & 2.275 & 0.297 & 6.2 \\
\hline 69.80 & 0.972 & 9.85 & 3.05 & 0.098 & 7.9 \\
\hline 70.64 & 0.983 & 7.15 & 2.5 & 0.340 & 6.4 \\
\hline 71.30 & 0.991 & 7.25 & 1.975 & 0.316 & 7.9 \\
\hline 72.04 & 1.001 & 7.15 & 2.35 & 0.234 & 6.3 \\
\hline 72.80 & 1.011 & 9.9 & 3.6 & 0.194 & 7.9 \\
\hline 74.30 & 1.030 & 9.25 & 4.075 & 0.104 & 6.6 \\
\hline 75.50 & 1.045 & 6.85 & 2.825 & 0.469 & 5.8 \\
\hline 76.33 & 1.056 & 7.1 & 2.275 & 0.187 & 6.6 \\
\hline 76.80 & 1.080 & 9.55 & 3.1 & 0.113 & 7.9 \\
\hline 77.80 & 1.096 & 9.5 & 3.5 & 0.200 & 7.8 \\
\hline 79.30 & 1.119 & 7.6 & 2.5 & 0.360 & 6.5 \\
\hline 80.10 & 1.132 & 7 & 2.3 & 0.217 & 6.5 \\
\hline 80.80 & 1.143 & 6.95 & 3.3 & -0.045 & 6.6 \\
\hline 81.15 & 1.148 & 7.5 & 2.25 & 0.311 & 6.6 \\
\hline 82.30 & 1.166 & 9.1 & 3.475 & 0.237 & 7.8 \\
\hline 83.84 & 1.190 & 10.05 & 3.5 & 0.129 & 7.7 \\
\hline 84.90 & 1.207 & 6.75 & 2.75 & 0.400 & 5.5 \\
\hline 85.79 & 1.221 & 10.3 & 3 & 0.083 & 7.9 \\
\hline
\end{tabular}

\begin{tabular}{|c|c|c|c|c|c|}
\hline $\begin{array}{l}\text { Depth } \\
\text { (mbsf) }\end{array}$ & $\begin{array}{l}\text { Age } \\
\text { (Ma) }\end{array}$ & $\phi 50$ & Sorting & Skewness & $\begin{array}{c}\text { Mode } \\
(\phi)\end{array}$ \\
\hline 87.30 & 1.244 & 9.75 & 3.325 & 0.203 & 7.9 \\
\hline 88.80 & 1.268 & 9.85 & 3.2 & 0.203 & 7.8 \\
\hline 90.30 & 1.291 & 10.4 & 3.15 & 0.111 & 7.9 \\
\hline 91.84 & 1.315 & 8.3 & 2.45 & 0.265 & 7.8 \\
\hline 92.90 & 1.332 & 9.3 & 2.55 & 0.157 & 7.9 \\
\hline 93.28 & 1.338 & 9.2 & 2.45 & 0.204 & 7.8 \\
\hline 94.10 & 1.351 & 7.75 & 2.525 & 0.228 & \\
\hline 95.56 & 1.374 & 10.15 & 3.225 & 0.163 & 7.9 \\
\hline 96.80 & 1.393 & 10.45 & 2.85 & 0.070 & 8.1 \\
\hline 97.10 & 1.398 & 7.05 & 3.4 & 0.074 & 6.6 \\
\hline 97.70 & 1.407 & 9.2 & 2.525 & 0.248 & 7.9 \\
\hline 98.30 & 1.417 & 8.5 & 2.25 & 0.289 & 7.9 \\
\hline 98.79 & 1.424 & 7.5 & 2.7 & 0.222 & 6.5 \\
\hline 99.20 & 1.431 & 8.2 & 2.525 & 0.327 & 7.8 \\
\hline 99.79 & 1.440 & 8.7 & 2.45 & 0.184 & 7.9 \\
\hline 101.32 & 1.464 & 7.9 & 2.7 & 0.278 & 6.6 \\
\hline 102.84 & 1.488 & 10.65 & 3.425 & 0.124 & 7.9 \\
\hline 104.29 & 1.510 & 10.6 & 3.475 & 0.137 & 8 \\
\hline 104.84 & 1.519 & 9.3 & 3.35 & 0.224 & 7.7 \\
\hline 106.29 & 1.542 & 10.75 & 3.7 & 0.095 & 8 \\
\hline 107.79 & 1.565 & 7.25 & 2.5 & 0.300 & 6.2 \\
\hline 109.29 & 1.589 & 10 & 3.525 & 0.149 & \\
\hline 110.50 & 1.607 & 7.8 & 2.45 & 0.265 & 6.7 \\
\hline 110.79 & 1.612 & 7 & 2.25 & 0.133 & 6.6 \\
\hline 111.10 & 1.617 & 7.35 & 2.55 & 0.353 & 6.8 \\
\hline 112.29 & 1.635 & 10.35 & 3.575 & 0.105 & 7.7 \\
\hline 113.29 & 1.651 & 9.6 & 3.375 & 0.156 & 7.5 \\
\hline 115.85 & 1.691 & 8.65 & 3.2 & 0.266 & 7.2 \\
\hline 117.28 & 1.714 & 9.8 & 3.55 & 0.155 & 7.7 \\
\hline 118.80 & 1.737 & 7.45 & 2.375 & 0.326 & 6.6 \\
\hline 120.31 & 1.761 & 10.3 & 3.275 & 0.038 & 8.2 \\
\hline 121.20 & 1.775 & 8.1 & 2.5 & 0.240 & 7.2 \\
\hline 121.80 & 1.794 & 9.95 & 2.875 & 0.061 & 8 \\
\hline 123.30 & 1.839 & 8 & 2.8 & 0.321 & 6.3 \\
\hline 123.86 & 1.856 & 7.8 & 2.675 & 0.346 & 6.4 \\
\hline 123.80 & 1.854 & 9.6 & 3.55 & 0.183 & 7.7 \\
\hline 125.30 & 1.899 & 8.4 & 2.675 & 0.271 & 7.4 \\
\hline 126.80 & 1.944 & 7.8 & 2.65 & 0.321 & 6.5 \\
\hline 128.30 & 1.970 & 8.7 & 2.675 & 0.178 & 7.8 \\
\hline 128.43 & 1.972 & 8.5 & 2.8 & 0.250 & 7.5 \\
\hline 129.87 & 1.996 & 8 & 2.6 & 0.288 & 6.7 \\
\hline 131.37 & 2.020 & 9.2 & 3.5 & 0.257 & 7.5 \\
\hline 132.87 & 2.044 & 10.1 & 3.4 & 0.147 & 8 \\
\hline 133.30 & 2.051 & 8.85 & 3.225 & 0.256 & 6.6 \\
\hline 134.80 & 2.075 & 8.25 & 2.575 & 0.301 & 6.8 \\
\hline 136.30 & 2.100 & 10.6 & 3.2 & 0.063 & 8.1 \\
\hline 137.30 & 2.116 & 10.2 & 3.1 & 0.097 & 7.9 \\
\hline 137.90 & 2.125 & 9.7 & 2.775 & 0.117 & 7.9 \\
\hline 138.49 & 2.135 & 10 & 3.175 & 0.071 & 8.2 \\
\hline 139.99 & 2.159 & 7.7 & 3.2 & 0.344 & 6 \\
\hline 142.79 & 2.204 & 8.8 & 3.05 & 0.279 & 7.4 \\
\hline 150.60 & 2.331 & 7.95 & 2.85 & 0.333 & 6.6 \\
\hline 160.19 & 2.485 & 7.65 & 2.875 & 0.374 & 6.3 \\
\hline 169.79 & 2.641 & 9.75 & 3.1 & 0.129 & 8 \\
\hline 179.43 & 2.721 & 8.9 & 3.425 & 0.270 & 7.5 \\
\hline 188.99 & 2.822 & 10.05 & 3.325 & 0.008 & \\
\hline 190.03 & 2.833 & 10.3 & 3.15 & 0.048 & 7.9 \\
\hline 190.50 & 2.838 & 9.35 & 3.25 & 0.123 & 7.6 \\
\hline 192.00 & 2.854 & 8.05 & 3.075 & 0.301 & 6.5 \\
\hline 193.06 & 2.865 & 8.4 & 3.1 & 0.258 & 6.3 \\
\hline 193.50 & 2.870 & 9.4 & 3.3 & 0.182 & 7.3 \\
\hline 194.43 & 2.880 & 10.05 & 2.55 & 0.118 & 8 \\
\hline 194.97 & 2.885 & 7.55 & 2.825 & 0.327 & 6.1 \\
\hline 195.84 & 2.895 & 8 & 2.7 & 0.259 & 6.8 \\
\hline 196.50 & 2.902 & 8.1 & 2.675 & 0.252 & 6.8 \\
\hline 197.50 & 2.912 & 10 & 3.25 & 0.077 & 8.1 \\
\hline 198.00 & 2.917 & 9.7 & 3.425 & 0.197 & 7.7 \\
\hline 198.70 & 2.925 & 8.3 & 3.375 & 0.274 & 5.9 \\
\hline
\end{tabular}




\section{C.J. Pudsey}

DATA Report: Grain-Size Data

Table T9 (continued).

\begin{tabular}{cccccc}
\hline $\begin{array}{c}\text { Depth } \\
(\mathrm{mbsf})\end{array}$ & $\begin{array}{c}\text { Age } \\
(\mathrm{Ma})\end{array}$ & $\phi 50$ & Sorting & Skewness & $\begin{array}{c}\text { Mode } \\
(\phi)\end{array}$ \\
\hline 200.08 & 2.939 & 8.45 & 3.175 & 0.228 & \\
201.58 & 2.955 & 9.6 & 3.45 & 0.188 & 7.6 \\
202.48 & 2.965 & 8.6 & 3.225 & 0.256 & 7.2 \\
203.12 & 2.972 & 8.4 & 3.275 & 0.298 & 6.6 \\
204.10 & 2.982 & 9.1 & 3.35 & 0.194 & 7.5 \\
204.59 & 2.987 & 10.1 & 3.025 & 0.091 & 8.2 \\
206.07 & 3.003 & 10.05 & 2.975 & 0.025 & 8.1 \\
207.57 & 3.019 & 8.4 & 3.2 & 0.203 & 5.9 \\
208.23 & 3.026 & 10.15 & 2.75 & 0.109 & \\
209.11 & 3.035 & 9.9 & 2.85 & 0.053 & \\
209.71 & 3.041 & 9.65 & 2.8 & 0.125 & 7.9 \\
211.18 & 3.057 & 9.5 & 2.6 & 0.115 & 7.9 \\
212.68 & 3.073 & 9.2 & 3.1 & 0.161 & 7.4 \\
214.22 & 3.089 & 9.05 & 2.9 & 0.190 & \\
215.73 & 3.105 & 8.5 & 2.9 & 0.241 & 6.8 \\
217.21 & 3.121 & 10.1 & 2.75 & 0.055 & 7.9 \\
\hline
\end{tabular}

Note: Numbers in italic are weak. 